\title{
Multiple Scattering of Light: Coherent Backscattering and Transmission
}

\author{
Ralf Lenke ${ }^{1,2}$ \\ Georg Maret ${ }^{1}$
}

${ }^{1}$ Universität Konstanz, Postfach 5560, D-78457 Konstanz, Germany

${ }^{2}$ Institut Charles Sadron (CRM-EAHP), 6 rue Boussingault, F-67083 Strasbourg Cedex, France 


\section{Contents}

1 Multiple Scattering of Light: Coherent Backscattering and Transmission 1 Introduction . . . . . . . . . . . . . . . . 1

1.1 Light propagation in single and multiple scattering samples . . . 5

1.1.1 The diffusion approximation . . . . . . . . . 7

1.1.2 Cross-over from single to multiple scattering . . . . . . 8

1.1.3 Influence of a boundary $\ldots \ldots \ldots \ldots$

1.2 Transmission geometry . . . . . . . . . . . . . . . . . . 14

1.2.1 Time-resolved transmission experiments . . . . . . . 17

1.2.2 Frequency-dependent speckle correlations . . . . . . . . 19

1.2.3 Diffuse photon-density waves . . . . . . . . . . . . . 21

1.3 Coherent backscattering of light . . . . . . . . . . . . 23

1.3.1 Theory of coherent backscattering . . . . . . . . . 2 25

1.3.2 Monte-Carlo simulations . . . . . . . . . . . . . . 38

1.3.3 Experimental setup of $\mathrm{CB} \ldots \ldots \ldots$. . . . . . 40

1.3.4 Examples of CB-cones . . . . . . . . . . . . 4 45

1.4 Appendix A: Transport mean free path $\ell^{\star} \ldots \ldots \ldots \ldots 5$

1.5 Appendix B: Proof of eqs. $(1.57,1.58) \ldots \ldots \ldots$

1.6 Appendix C: Monte-Carlo simulations . . . . . . . . . . 58

1.7 Appendix D: Divergent or convergent light source . . . . . . 60

1.8 Appendix E: Strongly inclined samples and refraction at the surface . . . . . . . . 61

$\begin{array}{ll}\text { References } & 65\end{array}$ 


\section{Glossary of Symbols}

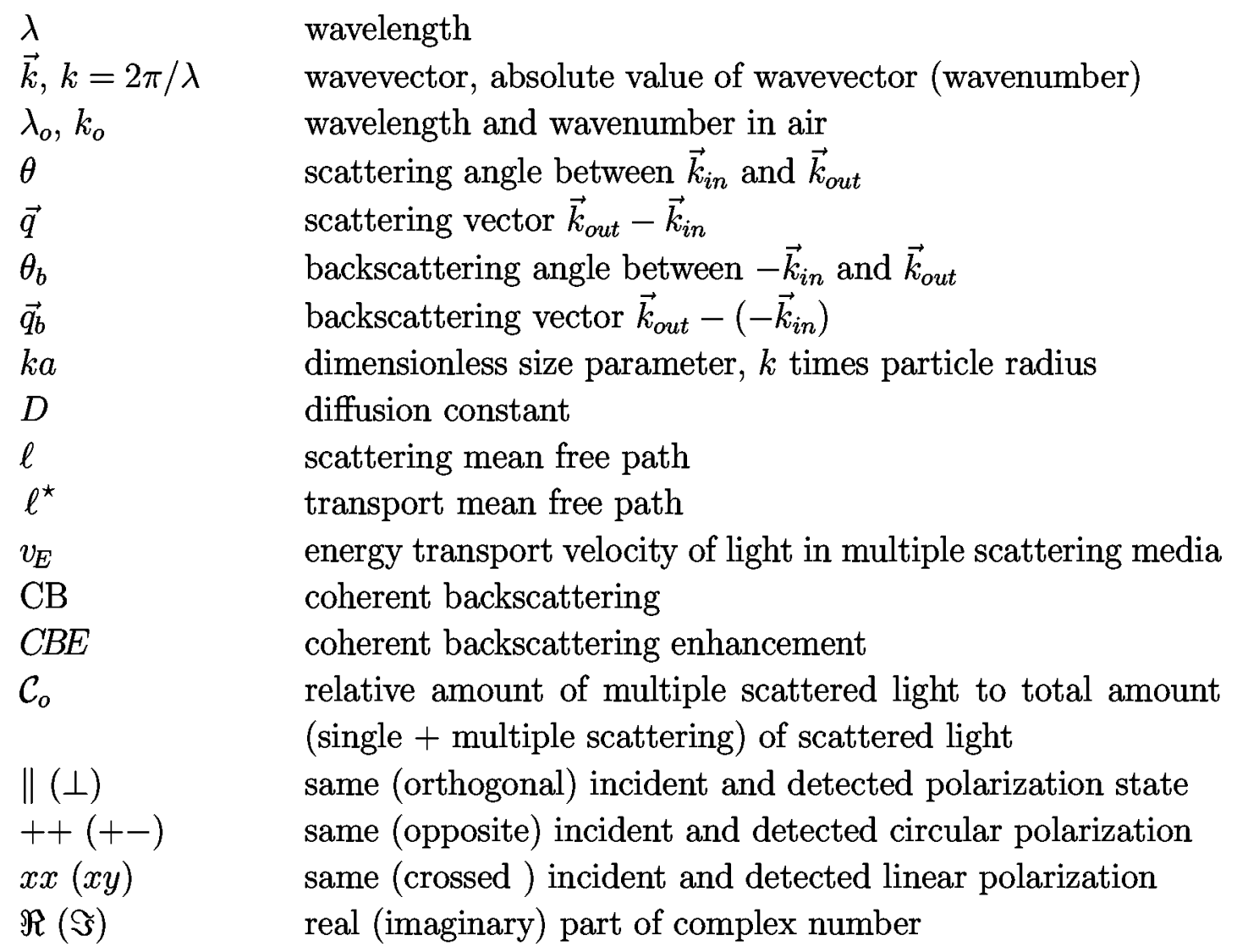




\section{Chapter 1}

\section{Multiple Scattering of Light: Coherent Backscattering and Transmission}

\section{Introduction}

In this chapter we discuss the propagation of light in multiple scattering media, i.e. in materials which appear white or turbid. These materials probably contribute the most common 'aggregate state' in nature, examples ranging from paint, paper, bio-materials, milk, rock to clouds. In multiple scattering samples, the incoming light is scattered successively numerous times, thereby rapidly losing the memory of the incident direction. After some scattering events, the propagation is a diffusion process. Consequently, the light emitted from the turbid media is also diffuse, the intensity varying only weakly with respect to the angle of observation (see fig. 1.2). The diffuse propagation of light, through a slab of a turbid media for example, is well understood since the beginning of the 19th century and is known as the Schwarzschild-Milne problem [1]-[3].

When using coherent incident light from a laser, a typical interference pattern, the so-called speckle pattern, is formed in the outgoing light (see fig. 1.1). This speckle pattern cannot be explained by the diffusion model of photons but is better described by a random walk of wavelets with amplitude and phase, as in Feynman's path model. Thus, the amplitude and phase of the outgoing waves are the result of the random superposition of all possible light paths in the sample. One finds [4] that, independent of the sample, the intensity distribution of the speckle spots is more or less exponential and that the average speckle spot size is proportional to the wavelength and inversionally proportional to the light emitting area. 
When the scatterers move, the light paths in the sample change, leading to rapid fluctuations in the speckle pattern. Thus the temporal fluctuations of the speckle pattern, i.e. the average, time-dependent intensity correlation function of one speckle spot, give information about the motion of the scatterers. With this novel, powerful technique, which is called quasi-elastic multiple light scattering or Diffusing Wave Spectroscopy (DWS), it is possible, for example, to determine the mean square velocity and thus the average particle size of the scatterers in the (liquid) sample.

In this chapter we will not consider aspects of this 'dynamic' multiple scattering for which the reader is referred to the current literature [5]. We focus on 'static' multiple scattering techniques, which are also applicable to solid or very viscous materials. The most promising and newest technique in this context makes use of the phenomenon of coherent backscattering (CB), which we will treat in greater detail. In fig. 1.2 the speckles are averaged out by moving the sample thus giving a more or less homogeneous scattering intensity. This is not quite correct in the backscattering geometry as can be seen in fig. 1.3. One observes a narrow, twofold enhancement of the multiply scattered intensity visible in the direction exactly opposed to the incident beam. It arises from the constructive interference between each light path and its reversed path. In the backscattering direction, both paths will always have the exact same length, independent of the movement of the scatterers. Consequently, due to this symmetry, there is an interference enhancement of the intensity by a factor of two. For angles deviating slightly from the precise backscattering direction, the intensity rapidly decreases to its diffuse value which is obtained by the incoherent summation of all light paths. The width of this so-called coherent backscattering cone is proportional to the wavelength times the 'optical thickness' $1 / \ell^{\star}$. The transport mean free path $\ell^{\star}$ corresponds to the 'sighting distance' of the turbid media. As $\ell^{\star}$ is always smaller than the light emitting area, the cone width is always larger than the average size of a speckle spot. However, a speckle spot can be much brighter than twice the average intensity. For a dense fog, $\ell^{\star}$ is about $30 \mathrm{~m}$, for paper it is about $10 \mu \mathrm{m}$ and for a good white paint it may be $1 \mu \mathrm{m}$. The width of the coherent backscattering cone of paper is about one degree, for a white paint 10 degrees.

The phenomenon of coherent backscattering is not restricted to light but may occur for any kind of waves. Moreover, it does not only show up outside the sample by the presence of the coherent backscattering cone but also inside a turbid media: Due to CB more light is scattered back to the light source than 
expected by a simple diffusion model of photons. Consequently, from the fact that each scatterer is a light source in itself to which a part of the light is scattered back, CB reduces the diffusion velocity in a turbid medium. This so-called weak localization of waves was found experimentally, for example in the electrical conductivity of semiconductors [6] and in the two-dimensional diffuse propagation of electrons on a liquid helium film, where the electrons are quasi-elastically scattered by helium atoms in the gas phase [7]. A strong localization was predicted by Anderson [8, 9] to occur in very strongly scattering media, where the wave is trapped by the disorder. The result is a metalinsulator transition as a function of the scattering strength. The interpretation of the electron localization as such is difficult due to the Coulombic interactions. Therefore, observation of the analogue in the case of electromagnetic waves would be very important and is a great matter of debate $[10,11]$ to this day. We will not enter this debate but refer the reader to the recent literature $[12,14]$, and restrict ourself to 'weakly' scattering materials (which include familiar turbid materials) and the phenomenon of the coherent backscattering cone. The CB-cone for light which was first observed in 1985 [15, 16], is central to 'modern' multiple light scattering physics. Apart from the fact that it is the precursor to localization phenomena, it reveals many other interesting aspects of multiple light scattering. It can be used for the characterization of turbid media. It can even be observed with sunlight, for example, in the brightness of the moons of Saturn and Jupiter. And it is partially related to the halo, seen in the so-called 'Glory' effect, which manifests itself in bright, coloured rings around ones own shadow in the sunlight scattered back from clouds or fog $[17,18]$.

In contrary to the CB-cone observed in backscattering geometry, localization phenomena are better observed in transmission geometry. In order to interprete them as such, a good understanding of 'classical' transmission theory is necessary. Therefore, for completeness, we will briefly summarize the principles of this theory. For 'non-classical' effects like localization phenomena or long-range speckle correlations the reader is referred to the current literature $[12,14]$. 


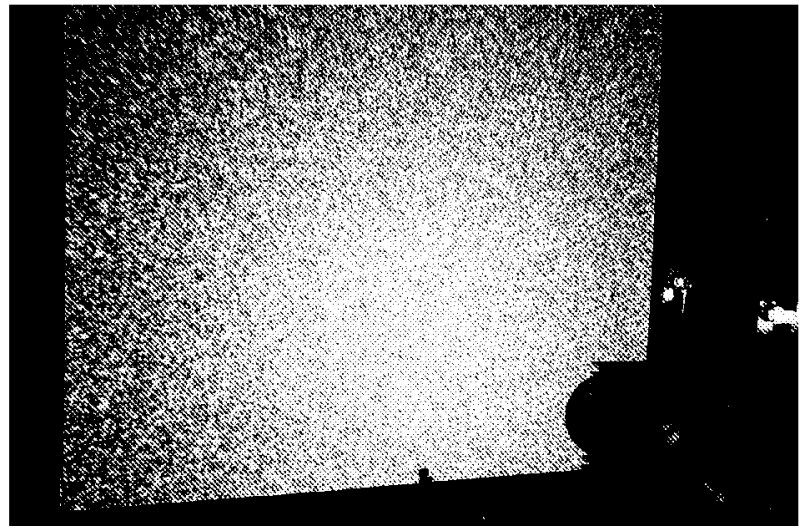

Figure 1.1: A typical speckle pattern produced by laser light scattered by a turbid sample. In fig. 1.2 no speckle pattern is visible because the sample was moved during the exposure time. Thus the speckles are averaged out in analogy to diffuse illumination.

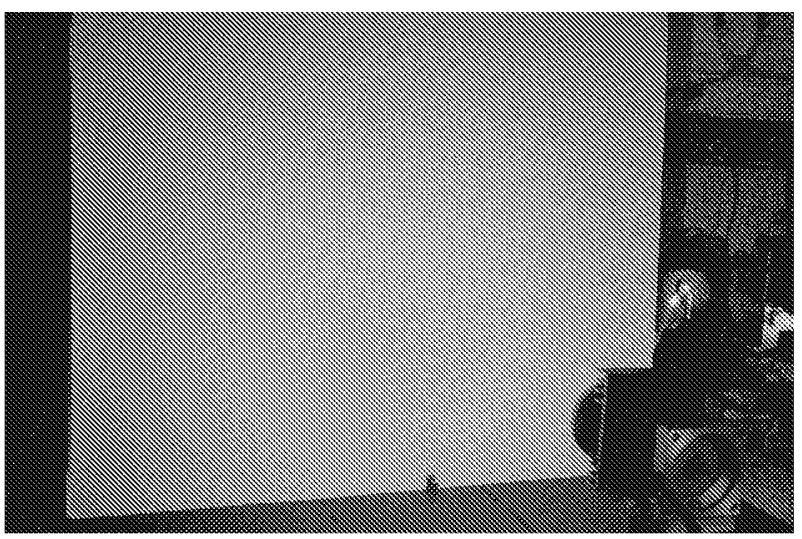

Figure 1.2 : Diffuse emitted light from a piece of paper. The intensity varies more or less according to Lambert's law: $I(\vartheta) \propto \cos (\vartheta), \vartheta$ being the angle of observation with respect to the normal at the surface.

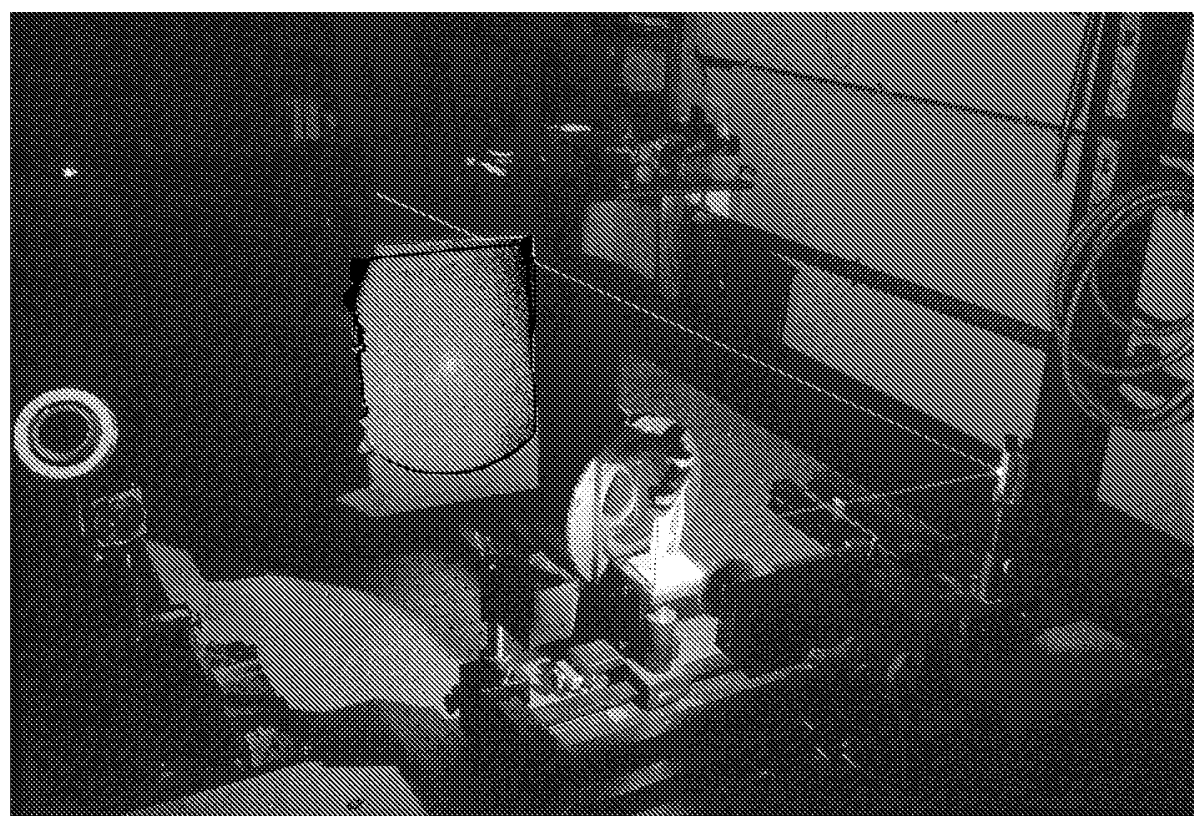

Figure 1.3 : Simplified experimental setup to observe coherent backscattering. Sample: Powder of microscopic crystals of $\mathrm{BaSO}_{4}$ which is rotated to destroy the speckles. Laser light is directed to the surface of the sample using a semi-transparent mirror. Part of the backscattered light passes through this mirror again, then a linear polarizer sheet and finally falls on a screen. The brighter spot in the centre of the backscattered light is the CB-cone.

Note: Figs. 1.1,1.3 are contrast enhanced in the black and white version. 


\subsection{Light propagation in single and multiple scattering samples}

In weakly scattering samples, the intensity $I(z)$ of a (plane) light wave is attenuated exponentially along the propagated distance $z$ because of scattering according to the Beer-Lambert law:

$$
\begin{aligned}
I(z) & =I(0) \exp (-z / \ell) \\
\ell & =1 / \rho_{N} \sigma_{\text {scat }}
\end{aligned}
$$

The characteristic length $\ell$ is called the scattering mean free path, $\rho_{N}$ is the number density of scatterers and $\sigma_{\text {scat }}$ the total scattering cross section. Consequently, the system is in the single scattering regime as long as $z \ll \ell$. Absorption brings in additional attenuation by a factor $\exp \left(-z / \ell_{a}\right), \ell_{a}$ being the average absorption length.

For $z \gtrsim \ell$ we have to distinguish between isotropic and anisotropic scattering. Rayleigh scattering, i.e. scattering from particles which are much smaller than the wavelength in the surrounding medium, is isotropic [19]. For scatterers, whose size is comparable to or larger than the wavelength, more light is scattered in the forward direction (normally). Anisotropic scattering of (spherical) particles is called Mie-scattering which reduces to Rayleigh-Gans-Debye scattering if the contrast of the scatterers with the surrounding medium is very small [19]. The anisotropy can be expressed by the terms

$$
\begin{aligned}
\langle\cos \theta\rangle_{\theta} & =\frac{\int \cos (\theta) \sigma(\theta) d \Omega}{\int \sigma(\theta) d \Omega} \text { or } \\
{\left[\langle 1-\cos \theta\rangle_{q}\right]^{-1} } & =\left[\frac{1}{2 k^{2}} \frac{\int \sigma(q) q^{3} d q}{\int \sigma(q) q d q}\right]^{-1},
\end{aligned}
$$

where $\theta$ is the scattering angle between incident and detected light, $k=2 \pi / \lambda$ and $q=\left|\vec{k}_{\text {out }}-\vec{k}_{\text {in }}\right|=2 k \sin (\theta / 2)$ are the absolute values of the wave and scattering vector, respectively. The average is taken over the differential cross section $\sigma(\theta),{ }^{1}$ i.e. the form factor of the scatterers. In addition, the ensemble of scatterers may have some structure, especially at higher volume fractions.

\footnotetext{
${ }^{1} \sigma(\theta)$ depends only on $\theta$, i.e. it is rotationally invariant about the incident direction because the incident light is considered to be non-polarized.
} 
In this case the average in eqs. $(1.3,1.4)$ has to be taken also over the structure factor of the medium [20].

In the multiple scattering regime, the diffuse light propagation can be described by a random walk model. A random walk is characterized by the mean square displacement $\left\langle|\vec{r}|^{2}\right\rangle=\left\langle x^{2}\right\rangle+\left\langle y^{2}\right\rangle+\left\langle z^{2}\right\rangle$ of the propagated distance. In one direction of the coordinate system, one finds for isotropic scattering:

$$
\begin{aligned}
\left\langle x^{2}\right\rangle & =n\left\langle(\triangle x)^{2}\right\rangle \\
& =\frac{2 n \ell^{2}}{d}=\frac{2 s \ell}{d}
\end{aligned}
$$

where $n$ is the number of steps of the random walk, $\left\langle(\Delta x)^{2}\right\rangle$ the mean square of the projection of the step length on the $x$-axis, $s$ is the path length and $d$ the dimensionality of the space in which the random walk takes place. The factor 2 in eq. (1.6) follows from the exponential step length distribution according to the Beer-Lambert law which is also valid inside a multiple scattering medium for each wavelet, after each scattering event. For anisotropic scattering, i.e. $\langle\cos \theta\rangle \neq 0$, one finds (in analogy with the radius of gyration of rigid polymers [21], see fig. 1.4):

$$
\begin{aligned}
\left\langle|\vec{r}|^{2}\right\rangle & =\left\langle\sum_{0 \leq i, j<n} \overrightarrow{\Delta r}_{i} \overrightarrow{\Delta r}_{j}\right\rangle=n\left\langle\overrightarrow{\Delta r}{ }^{2}\right\rangle+2\left\langle\sum_{i<j} \overrightarrow{\Delta r}_{i} \overrightarrow{\Delta r}_{j}\right\rangle \\
& =n\left\langle\overrightarrow{\Delta r}^{2}\right\rangle+2\langle\overrightarrow{\Delta r}\rangle^{2} \sum_{i<j}\langle\cos \theta\rangle^{j-i} \\
& =n\left\langle\overrightarrow{\Delta r}^{2}\right\rangle+2\langle\overrightarrow{\Delta r}\rangle^{2}\left(\frac{n\langle\cos \theta\rangle}{1-\langle\cos \theta\rangle}-\frac{\langle\cos \theta\rangle\left(1-\langle\cos \theta\rangle^{n}\right)}{(1-\langle\cos \theta\rangle)^{2}}\right) \\
& \approx n\left\langle\overrightarrow{\Delta r}^{2}\right\rangle+2\langle\overrightarrow{\Delta r}\rangle^{2} \frac{n\langle\cos \theta\rangle}{1-\langle\cos \theta\rangle},
\end{aligned}
$$

where we assume that the $\overrightarrow{\Delta r}$ ire independent and where we used $\left\langle\cos \left(\sum_{k=i+1}^{j} \theta_{k}\right)\right\rangle=\langle\cos \theta\rangle^{j-i}$. For an exponential step length distribution, one obtains with $\langle\overrightarrow{\Delta r}\rangle=\ell$ and $\left\langle\overrightarrow{\Delta r}^{2}\right\rangle=2 \ell^{2}$

$$
\begin{aligned}
\left\langle x^{2}\right\rangle=\frac{\left\langle|\vec{r}|^{2}\right\rangle}{d} & =\frac{2 n \ell^{2}}{d} \cdot \frac{1}{1-\langle\cos \theta\rangle}=\frac{2}{d} s \cdot \frac{\ell}{1-\langle\cos \theta\rangle} \\
& :=\frac{2\lrcorner \ell^{\star}}{d}=\frac{2 n^{\star} \ell^{\star 2}}{d}
\end{aligned}
$$


Now, compared to eq. (1.6), the scattering mean free path $\ell$ is replaced by a new characteristic length, the transport mean free path $\ell^{\star}=\ell /(1-\langle\cos \theta\rangle)$. At the same time the number of steps $n$ must be replaced by an effective number of steps $n^{\star}$ in such a way that the path length $s=n \ell=n^{\star} \ell^{\star}$ is unchanged. Consequently, for large $n^{\star}$, a random walk of $n$ correlated steps of average

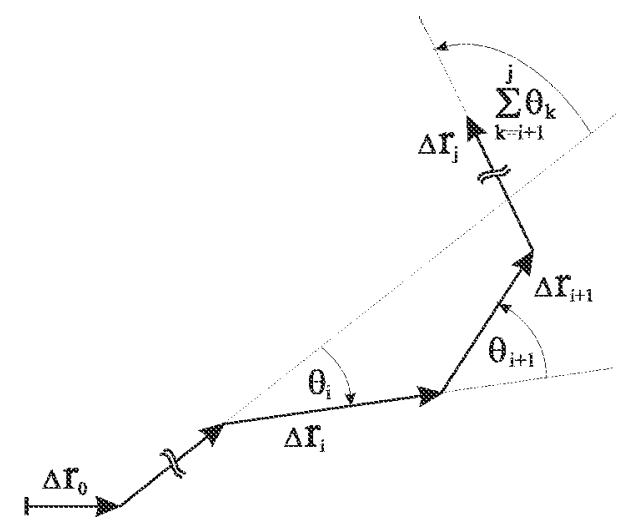

Figure 1.4 length $\ell$ can be replaced by a random walk of $n^{\star}$ non-correlated steps of average length $\ell^{\star}$. In the following we will use the symbol $\ell^{\star}$ when we refer to the transport mean free path, even if $\ell^{\star}=\ell$. We are now able to give a definition for the multiple scattering regime by $n^{\star}=s / \ell^{\star} \gg 1$.

\subsubsection{The diffusion approximation}

Another model used to describe multiple scattering is the diffusion approximation where the diffuse transport of energy is considered. The energy flux $\vec{\jmath}$ is given by $\vec{\jmath}=-D \vec{\nabla} \rho$, where $D$ is the diffusion constant and $\rho(\vec{r}, t)$ the energy density distribution. The light intensity $\vec{I}$ is given by the energy flux times surface area. The temporal variation of $\rho$ is given by $\partial_{t} \rho=-\vec{\nabla} \vec{\jmath}$. Consequently, the energy density distribution must follow the diffusion equation:

$$
\partial_{t} \rho(\vec{r}, t)=D \nabla^{2} \rho(\vec{r}, t)-\frac{1}{\tau_{a}} \rho(\vec{r}, t) .
$$

The second term describes absorption with the average characteristic absorption time $\tau_{a}$. Using the 'Ansatz' $\rho \propto \exp [i(k x-\omega t)]$, one obtains the general solution (for simplicity we restrict ourself to one dimension ${ }^{2}$ ):

$$
\rho(x, t)=\int_{-\infty}^{\infty} e^{-t / \tau_{a}} e^{-k^{2} D t}(A[k] \sin [k x]+B[k] \cos [k x]) d k .
$$

The free space Green function of the differential equation (1.9) for a diffusion which starts at $x=0$ follows immediately from eq. (1.10) as $\rho(x)=\rho(-x)$ and

\footnotetext{
${ }^{2}$ If $\rho$ is independent in $x, y$ and $z$-coordinates, the diffusion equation can be separated and we have $\rho_{\vec{r}}(t)=\rho_{x}(t) \rho_{y}(t) \rho_{z}(t)$. Due to the boundary conditions for $t \rightarrow \infty$, the separation constants must be zero.
} 
$\rho_{t=0}=\delta_{x=0}$. Thus, one obtains $A[k]=0$ and $B[k]=1 / 2 \pi$ and after integration:

$$
\rho(x, t)=\frac{\exp \left(-\frac{x^{2}}{4 D t}\right)}{\sqrt{4 \pi D t}} .
$$

This is a Gaussian distribution with the variance ${ }^{3}$

$$
\left\langle x^{2}\right\rangle=\int x^{2} \rho(x, t) d x=2 D t .
$$

Using eqs. $(1.12,1.8)$ we obtain the following relation between the diffusion constant and the transport mean free path:

$$
D=v_{E} \ell^{\star} / d
$$

$v_{E}=s / t$ being the energy transport velocity.

\subsubsection{Cross-over from single to multiple scattering}

Most explicit information about the scatterers is obtained by single scattering experiments. However, many materials are naturally in the multiple scattering regime and cannot be diluted for different reasons. Therefore, the aim of this chapter is to study what can be learned about the scatterers in the intermediate and multiple scattering regime. For $n^{\star} \gtrsim 10$ the diffusion approximation and the random walk model are equivalent. However, for $n^{\star} \lesssim 10$ the diffusion approximation is only a poor estimation. In the following we will discuss the random walk model for a small number of scattering events for isotropic scattering. For simplicity we restrict ourselves to one dimension. According to Beer-Lambert law (eq. 1.1), each step between two scattering events has an exponential step length distribution $p(x)=\exp (-x / \ell) / \ell$. Thus, after the first scattering event, the normalized intensity distribution is $\rho_{1}(x)=\exp (-|x| / \ell) / 2 \ell$. The intensity distribution after the second scattering event $\rho_{2}(x)$ is obtained by the probability to go from the origin to any point $\chi$ and from $\chi$ to the final point $x$ :

$$
\rho_{2}(x)=\int_{V} \rho_{1}(x-\chi) \rho_{1}(\chi-0) d \chi
$$

This corresponds to a convolution of a function with itself. As the two steps are independent of each other, one obtains with the convolution theorem for the Fourier transforms $\tilde{\rho}_{2}=\tilde{\rho}_{1}^{2}$. In one dimension, $\tilde{\rho}_{1}=1 /\left(1+k^{2} \ell^{2}\right)$ and thus

$$
\rho_{2}(x)=\frac{1}{2 \pi} \int_{-\infty}^{\infty} \frac{\cos (k x)}{\left(1+k^{2} \ell^{2}\right)^{2}} d k=\frac{1}{4} e^{-|x|}(1+|x|) .
$$

\footnotetext{
${ }^{3}$ It is $\left\langle\vec{r}^{2}\right\rangle=\left\langle x^{2}\right\rangle+\left\langle y^{2}\right\rangle+\left\langle z^{2}\right\rangle$.
} 
By analogy, one obtains for three scattering events with $\tilde{\rho}_{3}=\tilde{\rho}_{1}^{3}: \rho_{3}(x)=1 / 16$. $e^{-|x|}\left(3+3|x|+3 x^{2}\right)$ and so on. Only for more than about 10 scattering events $\left(1+k^{2} \ell^{2}\right)^{-n}$ can be approximated by the Gaussian $\exp \left(-n k^{2} \ell^{2}\right)$, for which the Fourier transform is also Gaussian and given by eq. (1.11). Consequently, only for $n \gtrsim 10$ the propagation is well described by the diffusion equation (1.9). For less than 10 scattering events a significant ballistic part in the propagation survives which is not described by the diffusion equation.

Experimentally, the multiple scattering regime is studied best in transmission through a sample which is many times thicker than $\ell^{\star}$. Of course, only informations about averaged quantities such as the transport mean free path $\ell^{\star}$, the transport velocity $v_{E}$ and the absorption length $\ell_{a}$ are obtained. If the sample has an anisotropic structure, $\ell^{\star}$ and $v_{E}$ may be different in different directions. One may measure the total intensity as a function of the sample thickness, as well as the time resolved intensity in a time of flight experiment of photons through the sample or, equivalently, the frequency correlation function. The intermediate regime is investigated by using thinner samples with thickness' of the order of $\ell^{\star}$. In this regime the intensity becomes an explicit function of the anisotropy of the scattering, i.e. the ratio $\ell^{\star} / \ell$, and the depolarization of the light. In fact, until now we have only considered 'scalar waves'. This is a good approximation in the multiple scattering regime as in three dimensions the state of polarization changes continuously along the scattering path. Normally, already after some scattering events the light is completely depolarized on average over all paths. On short paths, however, some degree of polarization may persist. The ratio $\ell^{\star} / \ell$ and the depolarization give some additional, (qualitative) information on the scatterers. However, the preparation of samples of thickness $\ell^{\star}$ may be difficult.

In the backscattering direction, contributions from the single, intermediate and multiple scattering regimes may be comparable even for very thick samples. The backscattering geometry may thus be more interesting in many cases as the preparation of thin slabs of the sample is avoided. However, quantitative interpretation of the measurements may be difficult due to the overlapping of the three regimes.

The multiple scattering regime is well described by the diffusion approximation. Another model would be the transport theory, using the Boltzmann equation which is a balance equation for the energy density and the flux into and out of a small volume element. In the following we will not discuss this approach, as this has already been done elsewhere (for literature see [22]) and 
as we will obtain the relevant results solely using the random walk model and a slightly modified diffusion approximation. The intermediate regime is better described by a general radiative transfer theory (for literature see [23, 24]) which, however, goes beyond the scope of this chapter. Moreover, this theory is more technical and gives no new physical insight. Instead, we use solely the random walk model, which is a powerful tool, especially when implemented with numerical simulations of the Monte-Carlo type. In the case of coherent backscattering, these simulations give particularly satisfactory results. They have the advantage that experimental parameters like sample geometry, laser beam profile, type of scatterers, surface effects and polarization can be included without difficulties.

\subsubsection{Influence of a boundary}

So far we have only considered light propagation in an infinite space. However, both the incident and the detected light have to cross the boundaries of the sample. Therefore a proper description of light transport near a surface is required. These surface effects, which are as yet poorly understood, may limit the quantitative interpretation of experimental data as the surface introduces, in a non-trivial

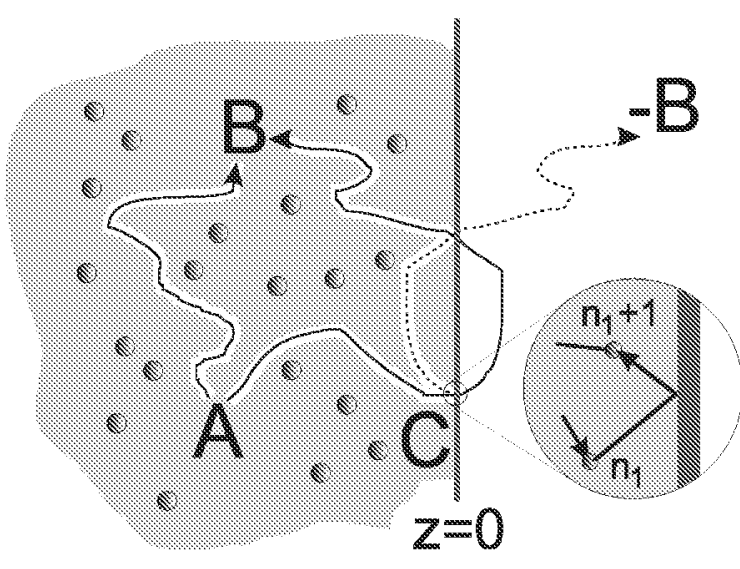

Figure 1.5 : reflecting surface way, additional scattering as well as internal reflections, i.e. reflections when the light wave impinges on the sample surface from the inside. In the following we will treat this problem in the diffusion approximation in order i) to get an analytic expression for the CB-cone later on in section 1.3.1. ii) to obtain an analytical expression for the transmission geometry which is more difficult to treat with simulations. Let us first consider a totally reflecting surface (fig. 1.5) located at $z=0$. In this case, the probability $\rho^{\prime}(A \rightarrow B)$ to go from $A$ to $B$ in the presence of a reflecting surface will be the free space probability $\rho(A \rightarrow B)$, given by eq. (1.11) with $z=B-A$, going from $A$ to $\mathrm{B}$, plus the 
probability going to the image point $-B$ :

reflecting surface: $\quad \rho^{\prime}(A \rightarrow B)=\rho(A \rightarrow B)+\rho(A \rightarrow-B)$.

The additional term corresponds to the paths that have been reflected an uneven number of times and whose mirrored paths would go to the mirror image of point $B$.

If the surface is absorbing or transparent, the probability to go from $A$ to $B$ is given by the free space probability to go from $A$ to $B$ minus the probability of all paths that pass outside of the sample (which is not a trivial function). As all these paths cross the surface, one could suppose that the probability of the paths that leave the sample is given by the probability to go

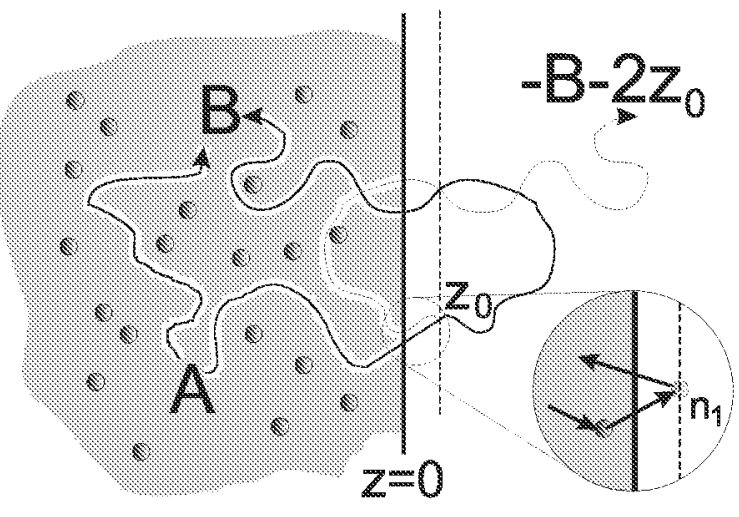

Figure 1.6 : open or absorbing surface from $A$ to any point $C$ on the surface and from there to $B$. As the probability to go from $C$ to $B$ is equal to the probability to go from $C$ to $-B$, one would finally find for the density distribution in presence of a transparent wall: $\rho^{\prime}(A \rightarrow B)=\rho(A \rightarrow B)-\rho(A \rightarrow-B)$. However, this is only a poor approximation as in this case, $\rho^{\prime}$ is zero at the surface. In addition there is an intensity flux out of the surface. This is a non-physical result as no flux can come from a region where the energy density is zero. In other words, the diffusion equation (1.9) is only reasonable if $\rho \gtrsim|\vec{\jmath}| / v_{E}$. The error is to assume that the random walk must hit the surface at some scattering event $n_{1}<n$ (more precisely $n^{\star}$ ). In reality, the random walk only crosses the surface between the scattering events $n_{1}$ and $n_{1}+1$. Consequently, the probability to go from $A$ to $B$ in $n$ steps in the presence of an open surface at $z=0$ is given by

$$
\text { open surface: } \quad \rho^{\prime}(A \rightarrow B)=\rho(A \rightarrow B)-\sum_{n_{1}+n_{2}=n} \rho\left(A \rightarrow z_{o}, n_{1}\right) \rho\left(z_{o} \rightarrow B, n_{2}\right),
$$

where $\rho$ is the free space probability and $z_{o}$ the first point of each random walk outside the sample. This is different to the case of reflection where a step of a random walk can be reflected at any point between the $n_{1}$ 'th and $\left(n_{1}+1\right)^{\text {'th }}$ 
event. Now we will propose an approximation which satisfies eq. (1.17). First we make the approximation that $\rho^{\prime}$ is independent in $x, y$ and $z$. Then, the $x$ - and $y$-components of $\rho^{\prime}$ are given by the free space probability (1.11) with $D t=n^{\star} \ell^{\star 2} / 3$. If $z_{o}$ is independent of $n_{1}$ and $n_{2}$, the $z$-component of the second term in eq. (1.17) can be approximated by:

$$
\sum_{n_{1}+n_{2}=n} \rho_{z}\left(A \rightarrow z_{o}, n_{1}\right) \rho_{z}\left(z_{o} \rightarrow B, n_{2}\right) \approx \int_{0}^{\infty} p\left(z_{o}\right) \rho_{z}\left[A \rightarrow\left(-B-2 z_{o}\right), n\right] d z_{o}
$$

where we have used the fact that the free space probability going from $z_{o}$ to $B$ is equal to the probability going from $z_{o}$ to $-B-2 z_{o}$ which is the image point of $B$ with respect to the surface at $z_{o}$. The function $p\left(z_{o}\right)$ is the normalized probability distribution of $z_{o}$. With the approximation that $p\left(z_{o}\right)$ and $\rho\left[A \rightarrow\left(-B-2 z_{o}\right)\right]$ are independent (what is justified if $n^{\star} \gtrsim 10$ ), the following expansion around the point $\bar{z}_{o}=\int z_{o} p\left(z_{o}\right) d z_{o}$ can be made:

$$
\begin{aligned}
& \int p\left(z_{o}\right) \rho_{z}\left[A \rightarrow\left(-B-2 z_{o}\right)\right] d z_{o}= \\
& \rho_{z}\left[A \rightarrow\left(-B-2 \bar{z}_{o}\right)\right]+\int p\left(z_{o}\right) O\left[z_{o}-\bar{z}_{o}\right]^{2} d z_{o}
\end{aligned}
$$

The linear term cancels as $\left\langle z_{o}-\bar{z}_{o}\right\rangle_{p\left(z_{o}\right)}$ is zero. The approximation is better the smaller $\left\langle\left(z_{o}-\bar{z}_{o}\right)^{2}\right\rangle_{p\left(z_{o}\right)}$ with respect to $\left(A+B+2 \bar{z}_{o}\right)^{2}$ and $n^{\star} \ell^{\star 2}$. Consequently, one obtains for the energy density distribution in the $z$-direction, in the presence of an open (or absorbing) surface at $z=0$ :

$$
\text { open surface: } \quad \rho_{z}^{\prime}(A \rightarrow B) \approx \rho_{z}(A \rightarrow B)-\rho_{z}\left[A \rightarrow\left(-B-2 \bar{z}_{o}\right)\right],
$$

where the function $\rho$ represents the free space propagation. The length $\bar{z}_{o}$ is the average penetration depth of the first step from the real sample into the space behind the open or absorbing surface. Now, the energy density distribution is zero at the point $\bar{z}_{o}$ in front of the surface which does not contradict the diffusion equation and the considerations made above. Often, $\bar{z}_{o}$ is expressed in units of the transport mean free path $\bar{z}_{o}=\gamma \ell^{\star}$. Assuming isotropic scattering and a constant intensity distribution inside the sample, one finds $\gamma=2 / 3$. The same value is obtained by the transport theory [22]. Assuming instead a linearly increasing intensity distribution inside the sample, which would vanish at a distance $\gamma \ell^{\star}$ in front of the surface, gives $\gamma=0.707$. This is very close to the 'exact' value ${ }^{4}$ of 0.7104 . However, since

\footnotetext{
${ }^{4}$ This problem is called the Milne problem, which describes diffuse transport through a slab.
} 
$\gamma$ depends on the intensity distribution close to the surface, the 'exact' value obviously depends on the experimental conditions, for example if the surface is partially reflecting. For a random walk in one dimension and isotropic scattering we find $p\left(z_{o}\right)=\exp \left(-z_{o} / \ell\right) / \ell$ and consequently $\gamma=1$ independent of the intensity distribution close to the surface. The reason for this is the self-similarity of the exponential function. Thus, in the one dimensional case, independent of the position of the $n_{1}$ 'th scattering event, the distribution of the distances $z_{o}$ of the $\left(n_{1}+1\right)$ 'th scattering event outside the sample from the surface, is always exponential.

It is not evident to what extent the value of $\gamma$ (besides its dependence on the intensity distribution close to the surface) also depends on the anisotropy of the scattering. However, by simulating (Monte-Carlo simulations) the intensity distribution inside a slab of thickness $L$ (according to curve 1 in fig. 1.7 , section 1.2), we did not find any differences up to an anisotropy $\ell^{\star} / \ell \leq 30$. Finally, one should realize that the parameter $\gamma$ does not follow from the diffusion equation but must be added 'by hand'. In addition to the problem of the best value for $\gamma$, in real samples one normally finds a non-trivial superposition of internal reflections and transmission events. Therefore, especially in the backscattering geometry, description of the problem in terms of eqs. $(1.16,1.20)$ is only a poor approximation. A better approach is given by the radiative transfer theory or by Monte-Carlo simulations. In both approaches the problem of $\gamma$ does not appear. 


\subsection{Transmission geometry}

If light is transmitted through a sample, usually an infinite slab of thickness $L \gg \ell^{\star}$ oriented in the $x, y$-plane, the light paths are rather long, i.e. $n=$ $s / \ell^{\star} \gg 1$, and the problem is well described by the diffusion approximation, while Monte-Carlo simulations become more difficult. With the 'image point technique' one obtains a series of image points (like a hall of mirrors) by multiple reflections with respect to both front and back of the sample.

Let us assume the situation in fig. 1.7, of a slab of a turbid medium of thickness $L$ between the points $z=\gamma \ell^{\star}$ and $z=\gamma \ell^{\star}+L$. In analogy with section 1.1.3, the diffuse intensity distribution should be zero at a distance $\gamma \ell^{\star}$ in front and behind the slab. In the following, for simplicity, we will set $\ell^{\star} \equiv 1$ and introduce the effective thickness $L^{\prime}=L+2 \gamma \ell^{\star}$. Now, the probability to go from $A$ to $B$ is the free space probability $\rho(A \rightarrow B)$ minus the probability to go to the two image points with respect to the surfaces at $z=0$ and $z=L^{\prime}$, i.e. to the points $-B$ and $2 L^{\prime}-B$. However, the probability to go to these image points is not the free space probability as in the case of a single surface but is the probability in the presence of the second, opposite surface and so on. Consequently, one obtains two series of image points which must be subtracted from $\rho(A \rightarrow B)$ :

$$
\begin{aligned}
\rho^{\prime}(A \rightarrow B)=\rho(A \rightarrow B) & \\
& -\left\{\rho\left(A \rightarrow B^{0}\right)-\left\{\rho\left(A \rightarrow B^{0 L^{\prime}}\right)-\left\{\rho\left(A \rightarrow B^{0 L^{\prime} 0}\right) \ldots\right\}\right\}\right\} \\
& -\left\{\rho\left(A \rightarrow B^{L^{\prime}}\right)-\left\{\rho\left(A \rightarrow B^{L^{\prime} 0}\right)-\left\{\rho\left(A \rightarrow B^{L^{\prime} 0 L^{\prime}}\right) \ldots\right\}\right\}\right\},
\end{aligned}
$$

where $B^{0}=-B$ and $B^{L^{\prime}}=2 L^{\prime}-B$ are the image points of $B$ with respect to the surfaces at $z=0$ and $z=L^{\prime}$, respectively. $B^{0 L^{\prime}}=2 L^{\prime}+B$ is the image point of $B^{0}$ with respect to $L^{\prime}$ and so on. Putting all terms together, one obtains:

$$
\rho^{\prime}(A \rightarrow B)=\sum_{m=-\infty}^{\infty} \rho\left(A \rightarrow B+2 m L^{\prime}\right)-\rho\left(A \rightarrow 2 m L^{\prime}-B\right) .
$$

Assuming that the $x, y$ and $z$-components are independent, one obtains in the $x$ - and $y$-directions the free space distributions $\rho_{x} \rho_{y}$ and in the $z$-direction:

$$
\rho_{z}^{\prime}(A \rightarrow B, t)=\frac{1}{\sqrt{4 \pi D t}} \sum_{m=-\infty}^{\infty}\left(e^{-\frac{\left(z_{B}+2 m L^{\prime}-z_{A}\right)^{2}}{4 D t}}-e^{-\frac{\left(2 m L^{\prime}-z_{B}-z_{A}\right)^{2}}{4 D t}}\right) .
$$

The sum converges quickly, as for $m$ larger than say \pm 2 the exponents are already very large. 


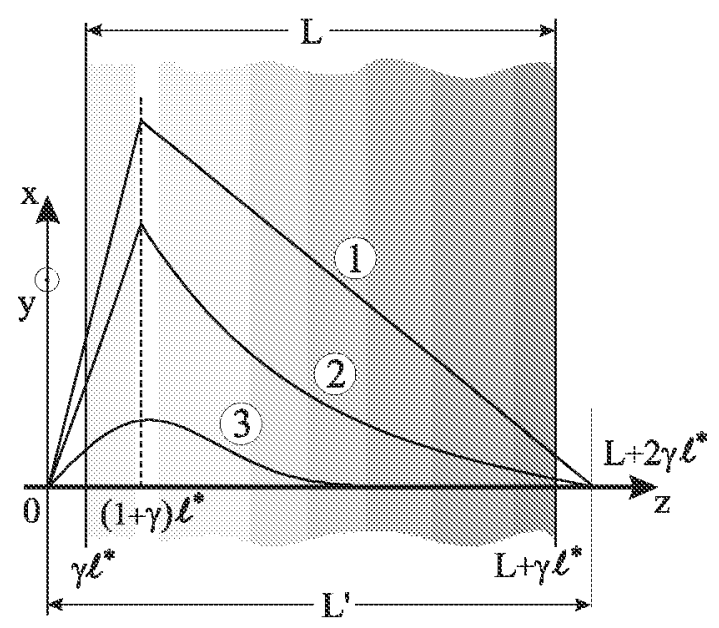

Figure 1.7: Diffuse transmission through a slab of thickness $L$ and infinite extension in $x, y$-directions. Curves 1)-3) are solutions of the diffusion equation with these boundary conditions, assuming that the diffuse flux starts at a depth $\ell^{\star}$ inside the sample. Curves 1) and 2) give the steady state intensity distributions, according to eq. (1.28) without and with absorption $\left(L_{a}=\right.$ $\left.3 \ell^{\star}\right)$, respectively, integrated over the $x, y$-directions. Curve 3) is the timedependent intensity distribution according to eq. (1.23) inside the sample after time $t=\ell^{\star 2} / D$, where $L_{a}=3 \ell^{\star}$.

The problem of the diffusion through a slab can also be solved directly using the diffusion equation (1.9). For this geometry, the general solution (1.10) reduces to (without absorption):

$$
\rho_{z}^{\prime}(t)=\sum_{n=1}^{\infty} e^{-\frac{n^{2} \pi^{2}}{L^{\prime 2}} D t} A_{n} \sin \left(\frac{n \pi}{L^{\prime}} z\right)
$$

With the condition that the intensity distribution at $t=0$ is a delta function at the point $A$, i.e. $\rho_{z}^{\prime}(t=0)=\delta\left(z-z_{A}\right)$, it follows that the factors $A_{n}$ have to be the elements of a Fourier row expansion of the delta function. Thus one obtains:

$$
\rho_{z}^{\prime}(A \rightarrow B, t)=\frac{2}{L^{\prime}} \sum_{n=1}^{\infty} e^{-\frac{n^{2} \pi^{2}}{L^{2}} D t} \sin \left(\frac{n \pi}{L^{\prime}} z_{A}\right) \sin \left(\frac{n \pi}{L^{\prime}} z_{B}\right)
$$

Both series are identical and can be transformed into each other by the Poisson sum formula. ${ }^{5}$ Absorption can be easily added by the additional factor $\exp \left(-t / \tau_{a}\right)=\exp \left(-s / \ell_{a}\right)=\exp \left(-D t / L_{a}^{2}\right)$, where $\ell_{a}=v_{E} \tau_{a}$ is the characteristic, 'microscopic' absorption length along the path and $L_{a}^{2}=D \tau_{a} \stackrel{3 D}{=} \ell_{a} \ell^{\star} / 3$ corresponds to a characteristic absorption distance in real space. The first

${ }^{5}$ A simplified version of the Poisson sum formula reads [25]:

$$
\sum_{n=-\infty}^{\infty} f(n)=\sum_{m=-\infty}^{\infty} \int_{-\infty}^{\infty} e^{-2 \pi m i a} f(a) d a
$$


equation (1.23) is more appropriate for calculating the time-dependent intensity distribution, whereas the second form (1.25) can be integrated and summed more easily in order to obtain the total, time and angle-integrated diffuse flux through the slab. This integration will be performed below. First, we integrate over $\rho_{x}$ and $\rho_{y}$ which gives only a factor 1 . The steady-state energy density (integrated over the $x$ - and $y$-directions) is obtained after integration of (1.25) over time:

$$
\rho_{z}^{t o t}:=\int d x \int d y \int d t \rho^{\prime}(A \rightarrow B)=\frac{2}{L^{\prime}} \sum_{n=1}^{\infty} \frac{\sin \left(\frac{n \pi}{L^{\prime}} z_{A}\right) \sin \left(\frac{n \pi}{L^{\prime}} z_{B}\right)}{D\left(\frac{1}{L_{a}^{2}}+\frac{n^{2} \pi^{2}}{L^{\prime 2}}\right)}
$$

This is the Fourier row expansion of [26]:

$$
\begin{aligned}
\rho_{z}^{t o t} & =\frac{L_{a}}{2 D} \frac{\cosh \left(\frac{L^{\prime}-\left|z_{B}-z_{A}\right|}{L_{a}}\right)-\cosh \left(\frac{L^{\prime}-z_{B}-z_{A}}{L_{a}}\right)}{\sinh \left(\frac{L^{\prime}}{L_{a}}\right)} \\
z_{B} \gg z_{A} & \frac{L_{a}}{D} \frac{\sinh \left(\frac{L^{\prime}-z_{B}}{L_{a}}\right) \sinh \left(\frac{z_{A}}{L_{a}}\right)}{\sinh \left(\frac{L^{\prime}}{L_{a}}\right)}
\end{aligned}
$$

Without absorption, i.e. for $L_{a} \rightarrow \infty, \rho_{z}^{\text {tot }}$ is triangular with the maximum value $\frac{\left(L^{\prime}-z_{A}\right) z_{A}}{D L^{\prime}}$ at $z_{A}$ (see fig. 1.7). Note, that $\rho_{z}^{\text {tot }}$ was integrated over the $x$ and $y$-directions, which corresponds to many experiments where the extension of the incident beam is several times larger than $L$. The time and angle integrated flux $I_{\text {tot }}$ through a slab of thickness $L$, which starts at the point $z_{A}$, is obtained according to the relations $\vec{\jmath}=-D \vec{\nabla} \rho^{\text {tot }}$ and $I_{\text {tot }}=\int \vec{\jmath} d x d y$ :

$$
I_{t o t}=-\left.D \frac{d \rho_{z}^{t o t}}{d z}\right|_{z=\gamma+L}=I_{o}\left(z_{A}\right) \frac{\cosh \left(\frac{\gamma \ell^{\star}}{L_{a}}\right) \sinh \left(\frac{z_{A}}{L_{a}}\right)}{\sinh \left(\frac{L+2 \gamma \ell^{\star}}{L_{a}}\right)}
$$

where we have replaced the effective thickness $L^{\prime}$ by the real thickness $L=$ $L^{\prime}-2 \gamma \ell^{\star}$ and multiplied all lengths by $1 / \ell^{\star}$ (at the beginning, $\ell^{\star}$ was set to 1 ). We should note that in the transmission geometry the precise values of $\gamma$ and $z_{A}$ are less important as both values are much smaller than $L$ and thus $I_{\text {tot }}(L)$ is only changed by a small constant factor. $I_{o}\left(z_{A}\right)$ is the intensity at the starting point of the diffusion. To a good approximation we can assume that the diffuse flux starts at a depth $\ell^{\star}$ inside the sample, so that $z_{A}=(\gamma+1) \ell^{\star}$. With this starting value, one obtains in the limit of zero absorption:

$$
\lim _{L_{a} \rightarrow \infty} I_{t o t}(L)=\frac{(1+\gamma) \ell^{\star}}{2 \gamma \ell^{\star}+L} \simeq(1+\gamma) \frac{\ell^{\star}}{L}
$$


This result resembles Ohm's law. The same result is obtained by the transport theory [22], where $\gamma=2 / 3$. With absorption, if $L \gg \ell^{\star}$ and $L \gg L_{a}$, one obtains from eq. (1.30) that the transmitted intensity is proportional to $\exp \left(-L / L_{a}\right)$. This justifies calling $L_{a}$ the characteristic absorption thickness.

\subsubsection{Time-resolved transmission experiments}

In analogy with eq. (1.30), the time-dependent, transmitted intensity $I(t)$ through a slab of thickness $L$ is obtained by differentiating eq. (1.23):

$$
I(t)=-\left.D \frac{d \rho^{\rho L^{\prime}}(t)}{d z}\right|_{z=L+\gamma} .
$$

Instead of this exact relation, the probability to go from one side of the slab to the other is used frequently in the literature, i.e. eq. (1.23) with $z_{A}=(\gamma+1) \ell^{\star}$ and $z_{B}=L+\gamma \ell^{\star}$ (see fig. 1.7). One can show that with these parameters $\rho^{\prime}(t)$ is proportional to a linear expansion of $I(t)$ :

$$
\rho^{\prime}(t)=\frac{(1+\gamma) \ell^{\star}}{D}\left(I(t)+O\left[\frac{\ell^{\star}}{L}\right]^{2}\right) .
$$

Of course the same relation is also valid between the total intensity $I_{\text {tot }}(L)$ and $\rho^{\text {tot }}$ of eqs. $(1.29,1.30)$. The most appropriate measuring technique to obtain a time of flight distribution is time-correlated single photon counting [32] where the sample is illuminated by a pulsed laser and the time of flight of single photons which succeed in crossing the slab is measured. As the laser system, we used a cavity-dumped dye-laser, pumped by a mode-locked $\mathrm{Ar}^{+}$-laser. This technique is appropriate as long as the samples are very turbid and thick. For larger values of $\ell^{\star}$ and thinner samples, where the transit times are shorter but the transmitted intensity is higher, streak camera measurements are preferable as they have a higher temporal resolution.

Fig. 1.8 shows a time of flight measurement, i.e. $I(t)$ through a slab of $\mathrm{TiO}_{2^{-}}$ powder. The experimental data were fitted using eqs. $(1.32,1.23)$. The fits are controlled by comparing the numerically integrated pulse shape with $I_{t o t}$, given by eq. (1.30). Thus, the diffusion constant $D$ and the absorption rate $\tau_{a}=L_{a}^{2} / D$ are obtained. Theoretically, using the relation $D=v_{E} \ell^{\star} / 3, \ell^{\star}$ can be obtained as well. However, especially in optical dense media, the energy transport velocity $v_{E}$ is not a well known parameter. Due to possible resonant scattering within the scattering particles, $v_{E}$ may be larger by about a factor 


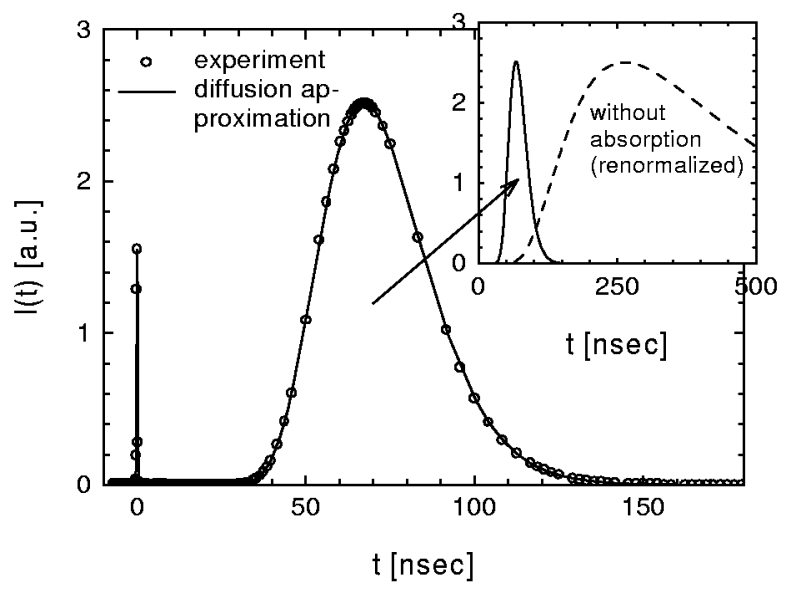

Figure 1.8: Dots: Time of flight measurement of photons through a slab of $\mathrm{TiO}_{2}$-powder (volume fraction 29\%). Sample thickness $7.38 \mathrm{~mm}, \lambda=0.580 \mu \mathrm{m}$. Solid lines: Several fits according to the diffusion equation. A fit without internal reflections at the surface gives $D=19 \mathrm{~m}^{2} / \mathrm{sec}$ and $L_{a}=0.688 \mathrm{~mm}$, independent of $v_{E}$ (we chose the two cases of $n=1.51$ and 2.7, obtaining $\ell^{\star}=0.287 \mu m$ and $0.514 \mu m, \ell_{a}=1.65 m$ and $0.92 \mathrm{~m}$ ). Assuming internal reflections of $70 \%$ (this corresponds to a mismatch of the index of refraction at the surface of $\left.\bar{n}_{\text {sample }} / n_{\text {air }} \approx 1.7\right)$ gives $D=20 \mathrm{~m}^{2} / \mathrm{sec}$ and $L_{a}=0.683 \mathrm{~mm}$. The spike at $t=0$, which is experimentally very difficult to suppress, corresponds to light which passed through cracks or around the sample. In time resolved measurements this fraction of light can be distinguished easily. In addition, it provides the time-resolution of the setup. For comparison, the inset shows the pulse shape if there would be no absorption.

of two than the velocity given by the average index of refraction [27]-[31]. On the other hand, on measuring $\ell^{\star}$ by static light scattering methods, i.e. total transmission measurements or coherent backscattering, $v_{E}$ can be determined. As already mentioned in section 1.2, for relatively thick samples, the time dependence of $I(t)$ is almost insensitive to $z_{A}$ and $\gamma$; only the total intensity is changed. However, there is a small dependence on internal reflections of the light as can be seen in the evaluation depicted in fig. 1.8. In order to include internal reflections in eq. (1.23), we assume that a fraction (re) of the light is reflected back into the sample and a fraction $(t r=1-r e)$ crosses the surface. By combining the method of multiple images with eqs. $(1.16,1.20)$ one obtains:

$$
\begin{aligned}
\rho_{z}^{\prime}(A \rightarrow B)= & \sum_{m, n=-\infty}^{\infty}\left\{\left(\begin{array}{c}
|2 m| \\
n
\end{array}\right)(-t r)^{|2 m|-n} r e^{n} \rho\left[z_{A} \rightarrow\left(2 m L^{\prime}+z_{B}-\operatorname{sign}[m] 2 n \gamma\right)\right]\right. \\
& \left.+\left(\begin{array}{c}
|2 m+1| \\
n
\end{array}\right)(-t r)^{|2 m+1|-n} r e^{n} \rho\left[z_{A} \rightarrow-\left(2 m L^{\prime}+z_{B}-\operatorname{sign}[2 m+1] 2 n \gamma\right)\right]\right\}
\end{aligned}
$$

For large times, i.e. in the decreasing part of the pulse, only the lowest Eigenvalue $n=1$ in eq. (1.25) survives. Consequently, the tail of the pulse decreases 
in proportion to $\exp \left[-\left(\frac{\pi^{2}}{L^{2}}+\frac{1}{L_{a}^{2}}\right) D t\right]$, where we have included absorption (which should not be too large). A. Genack has discussed this aspect more precisely in [13].

\subsubsection{Frequency-dependent speckle correlations ${ }^{6}$}

Alternatively to the time-of-flight measurements discussed in the previous section, the frequency-dependent intensity correlation function of the speckles can be measured. The speckle pattern is the consequence of the random superposition of all (multiply) scattered light paths in the detector. If the sample is illuminated with a single coherent beam, one normally observes two independent speckle patterns corresponding to the two orthogonal polarization states. Therefore, an analyzer must be put in front of the detector in order to observe one of the independent speckle patterns. The normalized intensity distribution of a speckle pattern is given by $p(I)=\frac{1}{\langle I\rangle} e^{-I /\langle I\rangle}$, where $\langle I\rangle$ is the average intensity. This follows from the facts that the distribution of the random light amplitudes $E$ is Gaussian and that $|E|^{2}=I$. The denominator in the exponential follows from the definition of the average intensity $\langle I\rangle=\int p(I) I d I$. The average angular width $\delta \alpha$ (in diameter) of a speckle spot depends on the expansion $W$ of the light emitting surface and the wavelength [33]: According to the van Cittert-Zernike theorem, the angular range $\delta \alpha$ within which the light coming from $W$ is coherent to an amount of $A$ per cent, is equal to the angle within which the amplitude of the diffraction pattern of a plane wave, emitted by the same area, has decreased from 1 (maximum) to $A$. The light coming from $W$ is supposed to be emitted from independent, quasi-monochromatic light sources which are equally distributed over the area $W$. Consequently, the 'coherence angle', i.e. the average width $\delta \alpha$ of the speckle spots which are produced by a round source, is equal to $1.22 \lambda / W$, which corresponds to the first zero of the diffraction pattern of a pinhole. Within a smaller range of $0.32 \lambda / W$, there is still a coherence of 88 per cent. The speckle spot size is given by the coherence angle times the distance from the source. For good speckle correlation measurements it is necessary to choose an angle of observation smaller than the coherence angle. This is best done with a monomodal fibre. When the scatterers move, the speckle pattern changes (rapidly) which, in the path model, is explained by the variation of the path configurations. In another model (which however is less advantageous experimentally) the

\footnotetext{
${ }^{6}$ We will treat this subject only briefly. A more detailed description is found in [13].
} 
fluctuations of the intensity of the speckle spots can be explained by a (very small) Doppler shift induced by the scattering of the moving particles. Consequently, the time-dependent autocorrelation measurements correspond to the spectroscopic wavelength measurements in Fourier space. In analogy, the timeof-flight measurements discussed above can also be performed in Fourier space, i.e. by frequency-dependent intensity correlation measurements of the speckles. This time, the scatterers do not move and the speckle intensity only changes due to the frequency shift. One obtains for the cross correlation function $\langle I(\omega) I(\omega+\Delta \omega)\rangle$ for the intensity of a speckle spot at the frequencies $\omega$ and $\omega+\Delta \omega$, in an average over many speckle spots and/or sample configurations:

$$
\begin{aligned}
& \langle I(\omega) I(\omega+\Delta \omega)\rangle=\left\langle E_{\omega} E_{\omega}^{*} E_{\omega+\Delta \omega} E_{\omega+\Delta \omega}^{*}\right\rangle= \\
& =\Re\left\langle\int_{s_{1-4}}\left|E\left(s_{1}\right) \| E\left(s_{2}\right)\right|\left|E\left(s_{3}\right)\right|\left|E\left(s_{4}\right)\right| \exp \left[i \frac{\omega}{v}\left(s_{1}-s_{2}\right)\right] \times\right. \\
& \left.\quad \times \exp \left[i \frac{\omega+\Delta \omega}{v}\left(s_{3}-s_{4}\right)\right] \exp \left[i\left(\phi_{s_{1}}-\phi_{s_{2}}+\phi_{s_{3}}-\phi_{s_{4}}\right)\right] d s_{1-4}\right\rangle .
\end{aligned}
$$

Each amplitude $E$ is represented by the path $s$ of a wavelet which has a probability proportional to $|E(s)|^{2}$. The average is taken over all possible combinations of the four light paths $s_{1-4}$. Here, we make the approximation that the scattering is independent of $\omega$ in the considered frequency range, i.e. $D(\omega)=$ constant! The phases $\phi_{\hat{\wedge}_{1-4}}$ correspond to possible phase shifts during the scattering events and to the phase shift introduced by the path from the source to the first and from the last scattering event to the detector. Averaging over all paths and configurations, i.e. all phase shifts $\phi_{s_{1-4}},\left(\frac{\omega}{v} s_{1,2}\right)$ and $\left(\frac{\omega+\Delta \omega}{v} s_{3,4}\right)$ results in the fact that the integrand must be multiplied with the following combination of delta functions $\delta\left(s_{1}-s_{2}\right) \delta\left(s_{3}-s_{4}\right)+\delta\left(s_{1}-s_{4}\right) \delta\left(s_{2}-s_{3}\right)$. In addition we have assumed that the phase shifts are independent of each other and that the possibility of crossed paths is negligible. Replacing in addition $v$ by $v_{E}$, the integral can be reduced to:

$$
\begin{aligned}
\langle I(\omega) I(\omega+\Delta \omega)\rangle & \propto\left\langle\int_{\diamond_{1,2}} p\left(s_{1}\right) p\left(s_{2}\right) d s_{1,2}\right\rangle+ \\
& +\Re\left\langle\int_{s_{1,2}} p\left(s_{1}\right) p\left(s_{2}\right) \exp \left[i \frac{\Delta \omega}{v_{E}} s_{1}\right] \exp \left[-i \frac{\Delta \omega}{v_{E}} s_{2}\right] d s_{1,2}\right\rangle
\end{aligned}
$$

where $p(s)$ is the intensity probability for a photon to travel along a certain light path $s$. In the following we assume that correlations between $s_{1}$ and $s_{2}$ 
are negligible. With $p(s)=p\left(v_{E} t\right)$, which corresponds to the probability $\rho^{\prime}(t)$ to go from one side of the slab to the other and the relation (1.33), $\rho^{\prime}(t) \propto I(t)$, one obtains:

$$
\langle I(\omega) I(\omega+\Delta \omega)\rangle=\langle I(\omega)\rangle^{2}+\left|\int I(t) \exp (i \Delta \omega t) d t\right|^{2}
$$

This is the relation between the frequency-dependent intensity correlation function and the absolute value of the Fourier transform of the time-dependent transmitted intensity $I(t)$. The integration can be performed in analogy with eqs. (1.25-1.29) with the replacement $1 / L_{a}^{2} \rightarrow 1 / L_{a}^{2}-i \Delta \omega / D$. For experimental comparisons between the correlation function and $I(t)$, see [13]. Of course, the sample and experimental setup need to be very stable, so that the speckle pattern does not change during the measuring time for a given frequency.

\subsubsection{Diffuse photon-density waves}

For the characterization of turbid media, diffuse photon-density waves [34] represent another, interesting method to study multiple light scattering, especially if the medium is less turbid. This method is also applicable in backscattering direction. If an oscillating source like an amplitude-modulated diode laser is used, the multiply scattered light will also oscillate to some extent. In this case, the source term $S$ and the solution of the diffusion equation (1.9) can be separated into a dc- and an ac-part: $S(\vec{r}, t)=\delta(\vec{r})\left[S_{d c}+S_{a c} \exp \left(-i \omega_{m} t\right)\right]$ and $\rho(\vec{r}, t)=\rho_{d c}(\vec{r})+\rho_{a c}(\vec{r}) \exp \left(-i \omega_{m} t\right), \omega_{m}$ being the modulation frequency. For the ac-part the diffusion equation takes the form of the Helmholtz equation [35]:

$$
\begin{aligned}
{\left[\nabla^{2}+k_{d i f f}^{2}\right] \rho_{a c}(\vec{r}) } & =\delta(\vec{r}) \frac{S_{a c}}{D} \\
\text { with } \quad k_{d i f f}^{2} & =\frac{i \omega_{m}}{D}-\frac{1}{L_{a}^{2}}=\frac{v_{E}}{D}\left[\frac{2 \pi i}{\lambda_{m}}-\frac{1}{\ell_{a}}\right]
\end{aligned}
$$


The solution is a highly damped (due to the diffuse propagation), spherical wave with the complex wave vector $k_{d i f f} ; \lambda_{m}$ is the wavelength of the modulation. The value of $k_{d i f f}$ in the complex plane is illustrated in the figure to the right. Without absorption, the real and imaginary parts are equal and the wavelength is $\lambda_{\text {diff }}=\sqrt{4 \pi D T_{m}}, T_{m}$ being the period of the modulation. With absorption, this wave-

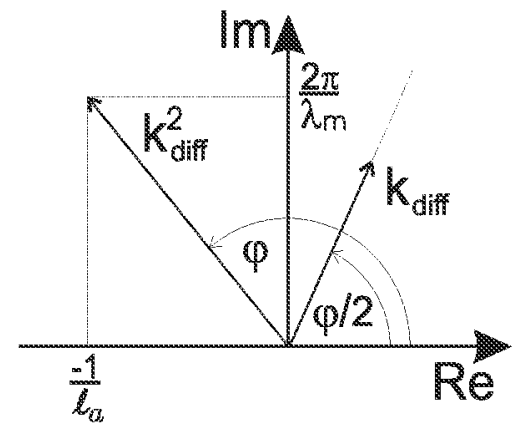
length as well as the imaginary part increases. Of course, with increasing absorption, the range of the photon cloud decreases rapidly. The relation between real and imaginary parts as a function of the modulation frequency, turbidity and absorption of the sample, determine the frequency and sample size range which can be explored reasonably. Despite the large damping, these diffuse photon-density waves have a well defined frequency, amplitude and phase which can be measured very precisely by lock-in techniques. Examples of applications can be found in [34]. 


\subsection{Coherent backscattering of light}

When averaging over speckles, the intensity of the light scattered from a multiple scattering sample depends only weakly on the scattering direction $\vartheta$ : With increasing angle of observation $\vartheta$ (with respect to the normal of the surface) the probability that the light leaves the sample from a depth $h$ without being scattered again decreases by $\exp \left(\frac{-h}{\ell^{\star} \cos \vartheta}\right)$. Integration over $h$ gives, to a first approximation, a $\cos (\vartheta)$-dependence of the emitted intensity (called Lambert's $\left.l a w^{7}\right)$. In addition to this $\cos (\vartheta)$-dependence, there may be a more or less pronounced (depending on the optical roughness of the surface) diffuse specular reflection of the incident light. To a first approximation, this reflection has the same properties with respect to direction and polarization dependence as the reflection from polished surfaces with the same 'average' index of refraction. However, as outlined in the introduction, a strong deviation from Lambert's law occurs in the exact backscattering direction (see fig. 1.3). In this direction one observes an intensity enhancement by a factor $\leq 2$. For the following reasons this brighter spot cannot just be a specular reflection or a speckle spot: i) Its direction does not depend on the orientation of the sample but rather on the direction of the incident light. ii) When using polarized incident light, the scattered light of this spot is polarized as well, whereas the surrounding intensity is mostly depolarized. Moreover, the backscattering peak has the same polarization as the incident light, especially in the case of circular polarization, whereas a reflection would have the opposite circular polarization. iii) In contrast to a speckle spot, its width is not inversely proportional to the width of the light emitting area but it is proportional to the optical density $1 / \ell^{\star}$ of the sample. In the majority of cases, the width of this so-called coherent backscattering cone is smaller than about one degree. In fig. 1.9 coherent backscattering versus Lambert's law is again illustrated.

Coherent backscattering has its origin in the constructive interference between each light path and its reversed path. In the exact backscattering direction $\left(\vec{k}_{\text {out }}=-\vec{k}_{i n}\right)$ both paths always have exactly the same length. Consequently, due to this symmetry, there is an interference enhancement of the intensity by a factor of two. For angles deviating slightly from the exact backscattering direction, the intensity rapidly decreases to the value which is obtained by the incoherent summation of all light paths. This is illustrated

\footnotetext{
${ }^{7}$ The exact $\vartheta$-dependence depends on the refraction at the surface, the intensity distribution close to the surface, the surface roughness or on the anisotropy of the scattering.
} 
Figure 1.9: Illustration of $C B$ versus Lambert's law. A multiple scattering sample is illuminated by a plane wave $\left(\vec{I}_{i n}\right)$. The 'sphere' represents the angular intensity distribution $I(\vartheta) \propto \cos \vartheta$ of the light which is scattered back from a turbid sample essentially according to Lambert's law, $\vartheta$ being the angle with respect to the normal of the surface. However, in the exact backscattering direction $\left(\theta_{b}=0\right)$, one additionally observes an intensity enhancement by a factor of $\leq 2, \theta_{b}$ being the angle with respect to the incident light. The width of the CB-cone is proportional

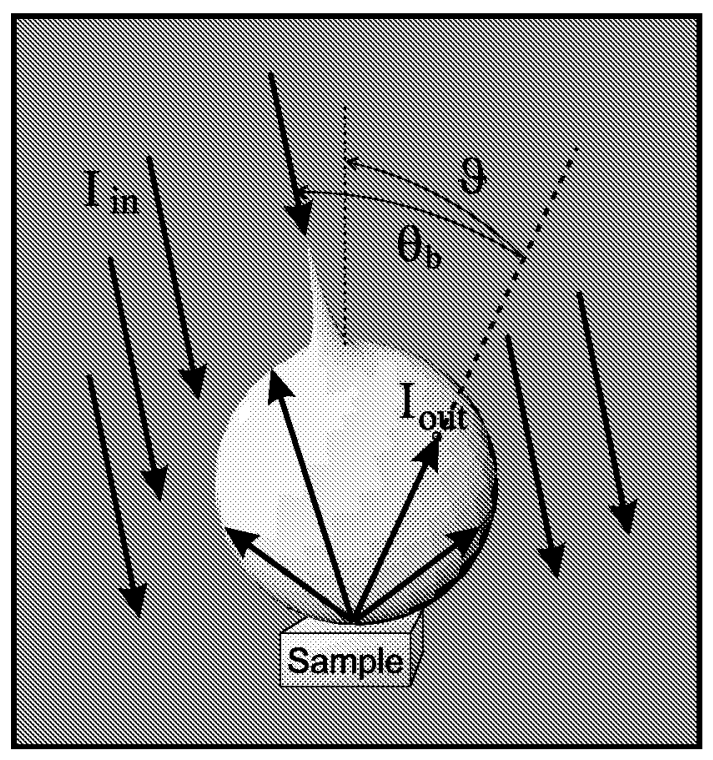
to the optical thickness of the medium and is most often very small. Here, it corresponds to a rather good white paint with $\ell^{\star}=2 \mu m$ at $\lambda=0.5 \mu m$.

and more explicitly explained in fig. 1.10. The reversed path exists if the direct path leaves the sample inside the coherence area of the light source.

Coherent backscattering is a very general phenomenon in nature every time multiple scattering of waves takes place. It is a very 'stable' effect, since it is not destroyed by the motion of the scatterers, nor by absorption or a short coherence length of the incident light. The latter will be shown in section 1.3.4 where the cone is detected with sunlight. Phenomena that destroy CB include magneto-optical Faraday rotation [36]-[40] (see section 1.3.4). Of course, coherent backscattering is also destroyed by non-elastic scattering, e.g. by fluorescence or by relativistic moving scatterers [36]. CB is closely related to the fact that light paths are reversible, i.e. "if I can see you, you can see me". However, strictly speaking, this is only completely correct for scalar waves. For electromagnetic waves, the polarization must also be considered. In fact, it emphasizes that $\mathrm{CB}$ is only completely satisfied for the same incident and detected polarization state. This follows from the theorem of reciprocity [41], which states that the scattering matrix of the reversed path is the transposed matrix of the direct path. We will study the influence of the polarization on CB in more detail.

The experimental setup used to measure coherent backscattering is rela- 


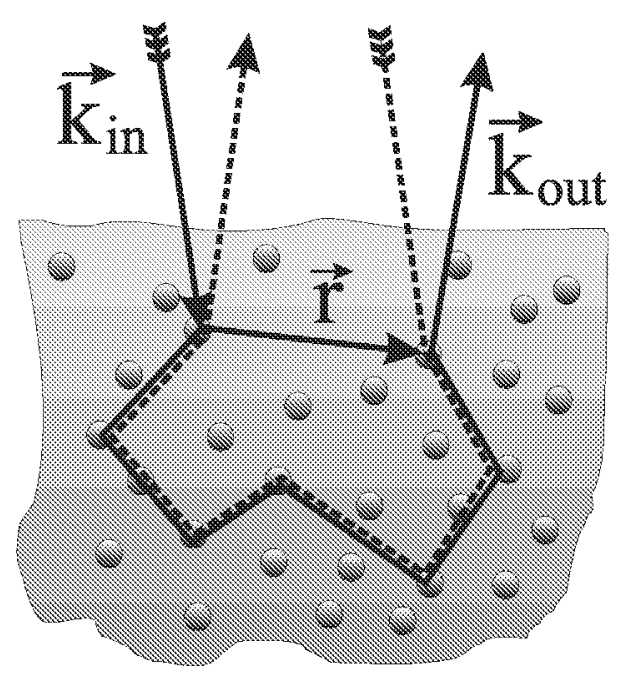

Figure 1.10: Illustration of the coherent backscattering effect: $C B$ is the constructive interference between each light path and its reversed path in the exact backscattering direction, $\vec{k}_{\text {out }}=-\vec{k}_{i n}$. In this direction, both paths have exactly the same length and the wavelets are in phase. In directions off exact backscattering a phase shift of $\vec{k}_{\text {out }} \cdot \vec{r}-\left(-\vec{k}_{\text {in }}\right) \cdot \vec{r}$ is introduced.

tively simple and qualitative and comparative studies of the optical density are very easy. However, like in all experiments in backscattering geometry, a quantitative evaluation is more complicated as the problem is not well described in the diffusion approximation. On the other hand, the shape of the cone, especially its dependence on the polarization of the light, not only contains the information of the optical density but also contains some information on the single scattering. We will only discuss here the 'physics' of the shape of the CB-cone. Quantitative evaluations are easily possible by Monte-Carlo simulations.

\subsubsection{Theory of coherent backscattering}

In the first part we will restrict ourselves to multiple scattering of scalar waves. This approach is sufficient to explain the general shape of the coherent backscattering cone as a function of the optical thickness and absorption. In the second part, we will consider the vector character of light and discuss the relationship between coherent backscattering and the polarization of light. We assume that there are no other interference effects in addition to coherent backscattering, i.e. that the speckle pattern has been completely averaged out by the motion of the scatterers and that the medium is completely random, without any (Bragg) reflections. For most cases, we will divide the ensemble of possible light paths into classes of paths of a certain length $s$, irrespective of their configuration. In section 1.3.2, in the context of Monte-Carlo simulations, this path model will be described in more detail.

Fig. 1.10 shows the situation for a light path with the distance vector $\vec{r}$ be- 
tween starting and end points and the particular associated path where the sequence of the scattering events is exactly reversed. In the exact backscattering direction these paths interfere constructively. For directions off exact backscattering, a difference in phase of $\vec{q}_{b} \cdot \vec{r}$, with $\vec{q}_{b}:=\vec{k}_{\text {out }}-\left(-\vec{k}_{i n}\right),{ }^{8}$ is introduced between the direct $(\otimes)$ and the reversed $(\odot)$ light paths. Accordingly, the intensity of one pair of paths oscillates as a function of the backscattering angle $\theta_{b}$, which is the angle between $\vec{k}_{\text {out }}$ and $-\vec{k}_{i n}$. However, the distance $\vec{r}$ is a broadly distributed quantity since $s$ is always (much) larger than $\ell^{\star}$. Thus, at large angles, as on average over all possible distances $\vec{r}$, the oscillations average out and the intensity corresponds to the incoherent sum of the contributions of all paths. The coherent backscattering enhancement $(C B E)$, i.e. the ratio of the coherent to the non-coherent summation of each pair of light paths, as a function of $\vec{q}_{b}$, is given by (for illustration see also fig. 1.11):

$$
C B E\left(\vec{q}_{b}, \vec{r}\right):=\frac{\left|e_{\otimes}+e_{\odot}\right|^{2}}{\left|e_{\otimes}\right|^{2}+\left|e_{\odot}\right|^{2}}=1+\frac{2 \Re\left[e_{\otimes} e_{\odot}^{*}\right]}{\left|e_{\otimes}\right|^{2}+\left|e_{\odot}\right|^{2}}=1+\cos \left(\overrightarrow{q_{b}} \cdot \vec{r}\right) .
$$

The complex numbers $e_{\otimes}$ and $e_{\odot}$ are the amplitudes of the direct and reversed paths, respectively; $\left({ }^{*}\right)$ denotes the complex conjugate. In the case of scalar waves, $e_{\otimes}$ and $e_{\odot}$ are equal in absolute values but different in phase, if $\vec{q}_{b} \neq 0$. If $|\vec{r}|$ is very small the oscillations become very broad. In fact, for $r \lesssim \lambda / 2 \pi$, $C B E$ is no longer distinguishable from Lambert's law. Normally, the probability of such almost closed light paths is negligible with the exception of single

${ }^{8} \mathrm{Using}-\vec{k}_{i n}$ here instead of $+\vec{k}_{i n}$, reflects the fact that CB acts to some extent like a phase conjugating mirror.

Figure 1.11: Solid gray curves: Angular dependent oscillations of the interference pattern for two different distance vectors $r=2 \mu m$ and $3.5 \mu m$ $(\lambda=0.5 \mu m, \vec{q} \| \vec{r})$. The width of the oscillations is inversionally proportional to $r$. All oscillations have their maximum at zero degree. At larger angles, however, many maxima and minima superimpose and by averaging over all paths, i.e. all possible distances

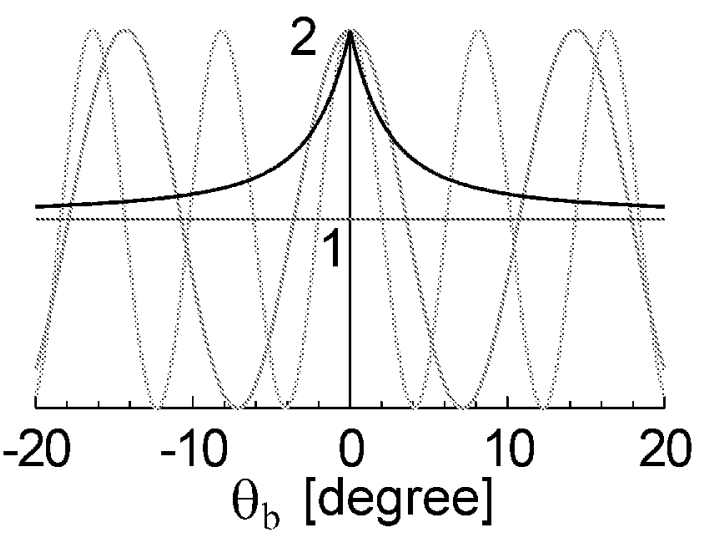
$\vec{r}$, the $C B$-cone decreases for angles $\gtrsim \lambda / 2 \pi \ell^{\star}$ to the average intensity. The bold solid curve corresponds to eq. (1.46) with $\gamma=0.7, \lambda=0.5 \mu \mathrm{m}$ and $\ell^{\star}=1 \mu \mathrm{m}$. 
scattering. For Rayleigh scattering for example, about 25 per cent of the light is scattered back by single scattering. For larger scatterers, the amount of single scattering decreases due to the anisotropy of the scattering. Consequently, single scattering (of Rayleigh scatterers) does not contribute to the coherent backscattering cone and reduces the enhancement factor below two. In other words, CB is a multiple scattering effect which contributes to the structure factor of the ensemble of the scatterers [42]. Single scattering is only responsible for the form factor but not for the structure factor. In the following we will denote the single and multiple scattered incoherent intensities by $I_{s s}$ and $I_{m s}$, respectively. Thus, the maximum value of the coherent backscattering is given by:

$$
C B E_{q_{b}=0}=1+\frac{I_{m s}}{I_{m s}+I_{s s}}:=1+\mathcal{C}_{o}
$$

Without absorption, the factor $\mathcal{C}_{o}$, which also depends on polarization, varies between 1 for large Mie spheres and $\approx 0.75$ for Rayleigh scattering and linear polarization. By using the same incident and detected circular polarization state, single scattering is normally suppressed as it flips one circular polarization state to the orthogonal one. With absorption, the short paths and especially single scattering are weighted more such that $\mathcal{C}_{o}$ becomes smaller.

To simplify the situation further, we will make the following additional assumptions i) near normal incidence, ii) $\theta_{b}$ and $q_{b}$ are very small and iii) the distance vector $\vec{r}$ lies approximately in the $x, y$-plane which is perpendicular to the incident light which propagates in the $z$-direction (see fig. 1.12). In this way, the reversed path is longer than the direct path by the distance $\Delta s=r_{x y} \sin \theta_{b} \approx r_{x y} \theta_{b}$ corresponding to a phase shift of $k_{o} \theta_{b} r_{x y} \approx q_{b} r_{x y}$ (with ii) follows $k_{o} \theta_{b} \approx q_{b}$, see Appendix E), where $k_{o}$ is the wave vector in air. Finally, on average over all distances $\vec{r}_{x y}$, i.e. integrated over the radial intensity distribution $I\left(\vec{r}_{x y}\right)$ which is the intensity distribution around

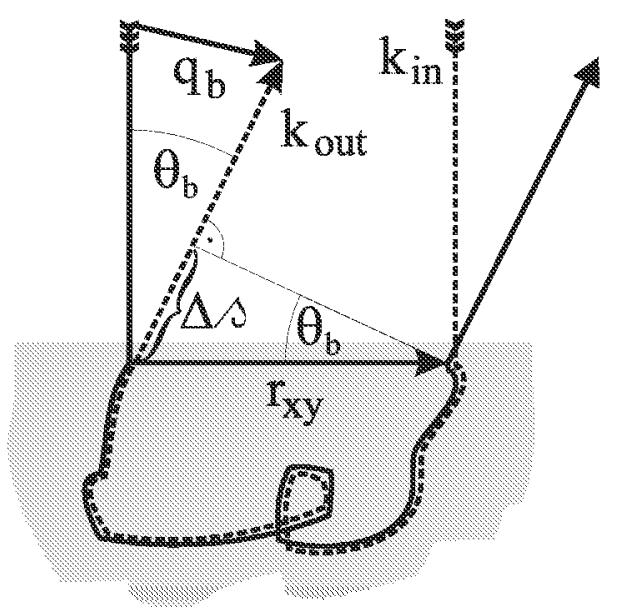

Figure 1.12: Simplified version of fig. 1.10 by the assumptions i)-iii) (see text). 
an illuminated, point like spot at $r_{x y}=0$, one obtains:

$$
\begin{aligned}
C B E\left(\vec{q}_{b}\right) & =1+\frac{1}{I_{m s}+I_{s s}} \int_{r_{x y} \approx \lambda_{o}}^{\infty} I\left(\vec{r}_{x y}\right) \cos \left(\vec{q}_{b} \cdot \vec{r}_{x y}\right) d \vec{r}_{x y} \\
I_{m s} & =\int_{r_{x y} \approx \lambda_{o}}^{\infty} I\left(\vec{r}_{x y}\right) d \vec{r}_{x y}, \quad I_{s s}=\int_{r_{x y}=0}^{\lambda_{o}} I\left(\vec{r}_{x y}\right) d \vec{r}_{x y}
\end{aligned}
$$

Consequently, the CB-cone is just the Fourier transform of the radial intensity distribution $I\left(\vec{r}_{x y}\right)$. Their widths are inversionally proportional to each other. $I\left(\vec{r}_{x y}\right)$ is obtained by measuring the spatial intensity distribution around an illuminated, point like spot, whereas $C B E\left(\theta_{b}\right)$ is obtained by measuring the angular intensity distribution when illuminating with a plane wave.

Within the diffusion approximation (see section 1.1.1), one finds for the radial intensity distribution $I\left(\vec{r}_{x y}, \diamond\right)=\frac{3}{4 \pi s \ell^{\star}} \cdot \exp \left(-3 r_{x y}^{2} / 4 s \ell^{\star}\right)$. Since the Fourier transform of this Gaussian is also a Gaussian, one obtains:

$$
C B E\left(\vec{q}_{b}\right)=1+\mathcal{C}_{o} \int_{s>0}^{\infty} p(s) e^{-\frac{1}{3} s \ell^{\star} q_{b}^{2}} d s
$$

where $p(\diamond)$ is the normalized path length distribution. Obviously, within the approximations made above, $C B E$ depends only on $\left|\vec{q}_{b}\right|$, i.e. it is symmetric by rotation around $\vec{k}_{i n}$. According to this, in most cases, we can angle-average the cone around its maximum value. It will be shown below that this is not quite correct in the case of linear polarization. The path length distribution $p(s)$ is obtained by the image method (see section 1.1.3). Apart from a normalization factor, one finds:

$$
\begin{aligned}
p(s) & \propto \frac{1}{\ell^{\star 2}} \int_{0}^{\infty} d z_{\text {in }} \int_{0}^{\infty} d z_{\text {out }} e^{-\frac{z_{\text {in }}}{\ell^{\star}}} e^{-\frac{z_{\text {out }}}{\ell^{\star}} \times} \\
& \sqrt{\frac{3}{4 \pi s \ell^{\star}}}\left[\exp \left(-\frac{3\left(z_{\text {in }}-z_{\text {out }}\right)^{2}}{4 s \ell^{\star}}\right)-\exp \left(-\frac{3\left(z_{\text {in }}+z_{\text {out }}+2 \gamma \ell^{\star}\right)^{2}}{4 s \ell^{\star}}\right)\right],
\end{aligned}
$$

where $z_{\text {in }}$ and $z_{\text {out }}$ are the depths of the starting and end points of a light path in the sample which are assumed to be exponentially distributed. The Gaussian terms in brackets correspond to the paths and image paths, respectively. With the approximation that $z_{\text {in }}=z_{\text {out }}=$ const. $=\ell^{\star}$, one obtains after integration over $s$ and normalization $[43,44]$ :

$$
C B E\left(q_{b}\right)=1+\mathcal{C}_{o} \frac{1-e^{-2(1+\gamma) \ell^{\star} \sqrt{q_{b}^{2}}}}{2(1+\gamma) \ell^{\star} \sqrt{q_{b}^{2}}}
$$


For small values of $q$ this curve can be approximated by

$$
C B E\left(q_{b}\right)=\left(1+\mathcal{C}_{o}\right)-\mathcal{C}_{o}(1+\gamma)\left|q_{b}\right| \ell^{\star}+O\left[q_{b}\right]^{2}
$$

Consequently, the top of the coherent backscattering cone has a triangular shape with a slope proportional to $\ell^{\star}$. For the half width of the cone one finds $\Delta q_{\frac{1}{2}} \approx 1 / \ell^{\star}$ or $\Delta \theta_{\frac{1}{2}} \approx \lambda / 2 \pi \ell^{\star}$. This corresponds to the uncertainty principle $(\Delta q \Delta r \approx 1)$ by assuming that the average value of $\Delta r_{x y}$ is about $\ell^{\star} .9$

According to eq. (1.44), the shape of the coherent backscattering cone can be interpreted as a superposition of Gaussian functions which become narrower the larger the path length $s$. At the same time, $p(s)$ decreases ${ }^{10}$ like $s^{\mathbf{- 3 / 2}}$, i.e. the weight of these Gaussians becomes smaller with increasing s. These effects combined result in the triangular cusp at $q_{b}=0$. Absorption introduces a cutoff length $\ell_{a}$ for the light paths which is added in eq. (1.44) by the factor $\exp \left(-s / \ell_{a}\right)$. That is equivalent to the replacement $q_{b}^{2} \rightarrow q_{b}^{2}+3 / \ell^{\star} \ell_{a}$. Note that the normalization factor in eq. (1.45) changes as well, such that $\int p(\diamond) d s \equiv 1$. Thus, one obtains for eq. (1.46) with absorption (for $\gamma=0.7$ ):

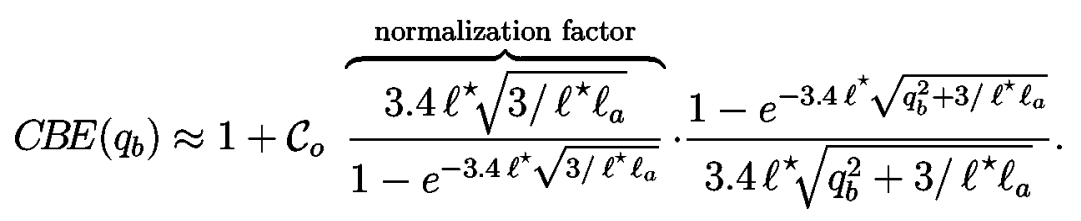

The normalization factor ensures that $C B E_{q_{b}=0}=1+\mathcal{C}_{o}$ has the same value as without absorption. Absorption does not lower $\mathrm{CB}$, since the amplitudes of the direct and reversed paths are equally affected. In other words, absorption acts on intensities (number of photons) and not on amplitudes of the light field. Nevertheless, $\mathcal{C}_{o}$ may depend on absorption if the relative amount of single to

${ }^{9}$ The approximation $z_{\text {in }}=z_{\text {out }}=$ const. $=\ell^{\star}$ is not really necessary as, in fact, eq. (1.45) can also be integrated directly (hint: After integration over $s$, there is a term $\exp \left[-\sqrt{\left(z_{1}-z_{2}\right)^{2}}\right]$ which must be integrated in $z_{2}$ from zero to $z_{1}$ and afterwards in $z_{1}$ from zero to infinity). The result is:

$$
\begin{aligned}
C B E\left(q_{b}\right) & =1+\mathcal{C}_{o} \frac{1+q_{b}-e^{-2 \gamma q_{b}}}{(1+2 \gamma) q_{b}\left(1+q_{b}\right)^{2}} \\
& =\left(1+\mathcal{C}_{o}\right)-\mathcal{C}_{o}(1+\gamma) \frac{1+\gamma}{\frac{1}{2}+\gamma}\left|q_{b}\right|+O\left[q_{b}\right]^{2} .
\end{aligned}
$$

However, despite the fact that this result is more correct than eq. (1.46), to our experience, it fits less well to experiments and simulations than eq. (1.46). Finally, both equations are valid only in the diffusion approximation.

${ }^{10}$ This can be seen by developing eq. (1.45) in $s^{-1}$, with $z_{\text {in }}=z_{\text {out }}=\ell^{\star}$. 


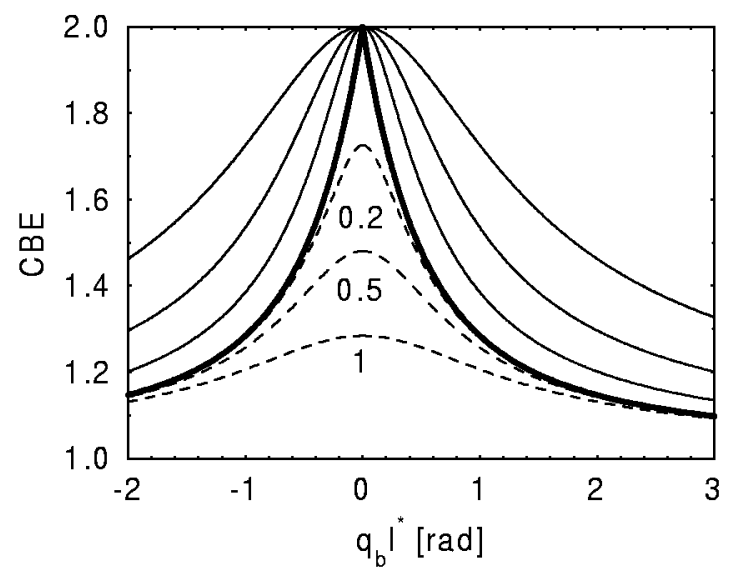

Figure 1.13: $\quad C B$-cones according to eq. (1.50). Bold solid line: without absorption. Dashed lines: With absorption $\left(\ell^{\star} / L_{a}=0.2 ; 0.5 ; 1\right)$, but the incoherent intensity is not corrected for absorption. Consequently, these curves demonstrate the cutoff of paths longer than about $\ell_{a}=3 L_{a}^{2} / \ell^{\star}$. Thin solid lines: These curves represent the real coherent backscattering cones, where also the incoherent intensity was corrected for absorption (same absorption values as dotted lines). Despite the rounding of the cone, absorption does not destroy $C B$, as it acts on the coherent and incoherent parts in the same way. Here, no single scattering was assumed.

multiple scattering varies with $\ell_{a}$. In analogy to the transmission geometry (section 1.2), the term $\sqrt{3 / \ell^{\star} \ell_{a}}=L_{a}^{-1}$ defines the direct space absorption length $L_{a}$. One can show that the curve shapes of a sample with absorption length $L_{a}$ and a sample of thickness $L=L_{a}$ are quite similar. ${ }^{11}$ In other words, paths which go deeper into the sample than about $L_{a}$ do not contribute to coherent backscattering. The consequence of the cutoff of paths longer than $\ell_{a}$ (on average) is, that no Gaussians narrower than about $q_{a}=L_{a}^{-1}$ contribute to the coherent backscattering cone in eq. (1.44). Consequently the cone is rounded off below $q_{a}$. In fig. 1.13 cones with different absorption lengths are plotted according to eq. (1.50).

However, as already mentioned in section 1.1, the diffusion approximation gives only a rough estimation of the real path length distribution in backscattering direction. In reality, $p(s)$ depends for example on the type of scattering, especially if it is isotropic or not, the mismatch of the index of refraction at the surface and the depolarization of the light. The diffusion approximation is useful for a qualitative understanding of coherent backscattering. For quantitative evaluations, however, numerical simulations of Monte-Carlo type (see Appendix C) are much more useful.

\footnotetext{
${ }^{11}$ The cone shape for a slab of thickness $L$ can be calculated by using eq. (1.23) instead of eq. (1.20).
} 
In the next part we discuss the effects of the vector character of light on coherent backscattering. Despite the fact that in exact backscattering direction the direct and reversed paths have exactly the same length, the existence of a constructive interference is not evident, as it requires total correlation between the polarization of the light for the direct and reversed paths, although multiple scattering depolarizes. In order to describe the multiple scattering of vector waves, it is useful to introduce a local (right handed) coordinate system for each scattering event, where the $z$-axis always points along the direction of propagation. The transformation of the local coordinate system from the (i1)'th scattering event to the next, corresponds to a rotation of the (i-1)'th scattering plane, which is built up by $\vec{k}_{i-1}$ and $\vec{k}_{i}$, into the i'th scattering plane, built up by $\vec{k}_{i}$ and $\vec{k}_{i+1}$. In the far field approximation, i.e. $\ell \gg \lambda$, the light amplitude can always be assumed perpendicular to the direction of propagation $\vec{k} /|k|$. Consequently, in these local coordinate systems, each light path can be described by a $2 \times 2$ matrix $M$ which is a product of $2 \times 2$ matrices:

$$
\mathrm{M}=\mathrm{R}_{n} \mathrm{~S}_{n} \ldots \mathrm{R}_{2} \mathrm{~S}_{2} \mathrm{R}_{1} \mathrm{~S}_{1}
$$

where $\mathrm{R}_{i}$ are rotational matrices which fulfill the transformation from one local coordinate system to the next. The matrices $S_{i}$ describe the single scattering events. For spherical scatterers, they are diagonal but complex and depend only on the scattering angles $\theta_{i}[19]$. For Rayleigh scattering they are real and proportional to $\left(\begin{array}{cc}1 & 0 \\ 0 & \cos \theta\end{array}\right)$. Therefore, for the reversed path, the matrices $\mathrm{S}_{i}$ for Rayleigh scattering are the same. On the other hand, on the reversed path, the light experiences the inverse transformations of the local coordinate systems. Thus, with $R^{-1}=R^{\top}$ and $S^{\top}=S$, the total scattering matrix for the reversed path is $M_{\odot}=S_{1} R_{1}^{\top} S_{2} R_{2}^{\top} \ldots S_{n} R_{n}^{\top} \equiv M_{\otimes}^{\top}$, which is just the transposed matrix of the direct path. ${ }^{12}$ The fact that the scattering matrix for the reversed path is just the transposed matrix of the direct path is known as the theorem of reciprocity [41]. It follows directly from Maxwell's equations and is therefore not restricted to Rayleigh scattering. Consequently, $M_{\otimes}$ equals $M_{\odot}$ as long as $M$ is symmetric (i.e. $M^{\top}=M$ ). However, $M$ is not symmetric, in general, due to the presence of the matrices $R$, which reflect torsions of the light path in three-dimensional space. Only for light paths traveling in one plane, the rotations $R$ are equal in identity and $M$ is symmetric (if the $S_{i}$ are symmetric). The importance of these particular light paths will be discussed further below.

\footnotetext{
${ }^{12}$ In this notation, the coordinate system on the reversed path is the same as on the direct path, i.e. on the reversed path, the light propagates opposite to the $z$-direction!
} 
Figure 1.14: Theorem of reciprocity: $\vec{P}$ polarizer, $\vec{A}$ analyzer, $\mathrm{M}$ represents a multiple scattering light path which is passed in direct and reversed directions. In this configuration, the complex amplitude $e_{\otimes}$ of the light which passes on the direct path polarizer $\vec{P}$, sample $\mathrm{M}_{\otimes}$ and analyzer $\vec{A}$, is equal to the amplitude $e_{\odot}$ of the reversed path $\left(\vec{A} \rightarrow \mathrm{M}_{\odot} \rightarrow\right.$ $\vec{P}) . \vec{P}$ and $\vec{A}$ can be any polarizer, i.e. linear, circular etc. Before passing $\vec{P}$ and $\vec{A}$ and after having passed

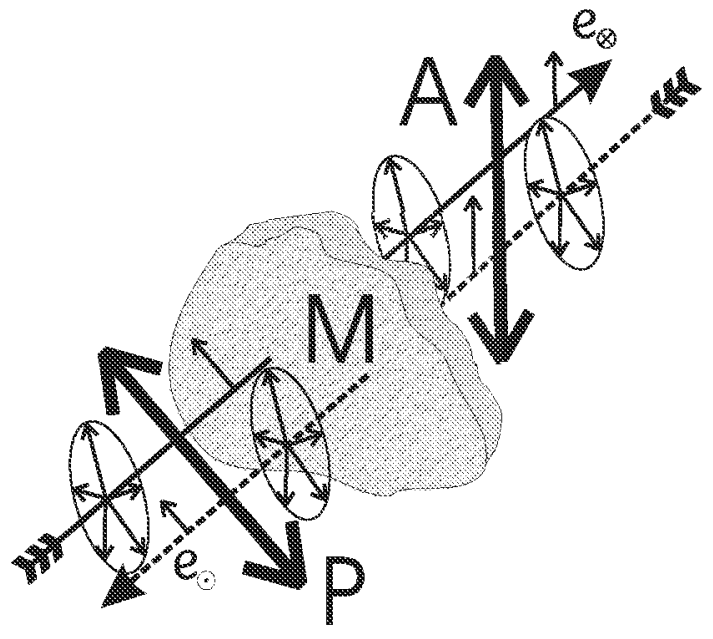
the sample, the light is depolarized.

The theorem of reciprocity $\left(M_{\odot}=M_{\otimes}^{\top}\right)$ is equivalent to the following relationship between the complex amplitudes $e_{\otimes}$ and $e_{\odot}$ of a given polarization state on the direct and reversed paths (see fig. 1.14): The amplitudes and phases of the direct and reversed paths are the same if the incident and detected light is completely polarized in one polarization state and if the incident polarization of the direct path is identical to the detected polarization of the reversed path and vice versa. However, the incident and detected polarization states need not to be the same. The equivalence between this statement and the theorem of reciprocity can be verified easily by using the polarizer/analyzer pairs $[\vec{P}, \vec{A}]=\left[\left(\begin{array}{l}1 \\ 0\end{array}\right),\left(\begin{array}{l}1 \\ 0\end{array}\right)\right] ;\left[\left(\begin{array}{l}0 \\ 1\end{array}\right),\left(\begin{array}{l}0 \\ 1\end{array}\right)\right] ;\left[\left(\begin{array}{l}1 \\ 0\end{array}\right),\left(\begin{array}{l}0 \\ 1\end{array}\right)\right] ;\left[\left(\begin{array}{l}0 \\ 1\end{array}\right),\left(\begin{array}{l}1 \\ 0\end{array}\right)\right]$, any matrix $\mathrm{M}=\left(\begin{array}{ll}a & b \\ c & d\end{array}\right)$ and the general definitions for the correlated amplitudes for the direct and reversed paths: $e_{\otimes}^{\|}=\vec{A} \mathrm{M}_{\otimes} \vec{P}, e_{\odot}^{\|}=\vec{P} \mathrm{M}_{\odot} \vec{A}$. In other words, the argument: "if I can see you, you can see me" is strictly speaking correct only if both observers stand behind a polarizer sheet. In the following we choose $\vec{P}=\vec{A}$, i.e. $e_{\otimes}^{\|}=\vec{P} \mathrm{M} \vec{P}$ and $e_{\odot}^{\|}=\vec{P} \mathrm{M}^{\top} \vec{P}$. We will denote this case as parallel polarized state $(\|)$, independent if the light is, for example, linear $(x x)$ or circularly $(++)$ polarized.

As a consequence, the considerations made above for coherent backscattering and scalar waves remain applicable as long as the incident and detected polarization states are the same.

For orthogonal polarization states, however, the theorem of reciprocity does not provide any particular correlation between the direct and reversed paths, 
and consequently gives no coherent backscattering cone to a first approximation. Nevertheless, some correlation is possible in the crossed channels as well, due to the presence of short light paths. This correlation is closely related to the depolarization of the light. In fact, a relation exists between the height of the coherent backscattering cone in the crossed polarized channels and the degree of depolarization of the light. The crossed polarized states are generally defined as: $e_{\otimes}^{\perp}=\vec{A} \mathrm{M} \vec{P}$ and $e_{\odot}^{\perp}=\vec{P}^{\perp} \mathrm{M}^{\top} \vec{A}^{\perp} \cdot{ }^{13}$ However, in the following we choose $\vec{P} \perp \vec{A}$, i.e. $e_{\otimes}^{\perp}=\vec{P}^{\perp} \mathrm{M} \vec{P}$ and $e_{\odot}^{\perp}=\vec{P}^{\perp} \mathrm{M}^{\top} \vec{P}$. The special cases of orthogonal linear and orthogonal circular polarization will be denoted by $(x y)$ and $(+-)$, respectively. The degree of linear and circular polarization, $\mathcal{P}_{l}$ and $\mathcal{P}_{c}$, which survives the multiple scattering is defined as:

$$
\mathcal{P}_{l}=\frac{I_{i n c}^{x x}-I_{i n c}^{x y}}{I_{i n c}^{x x}+I_{i n c}^{x y}}, \quad \mathcal{P}_{c}=\frac{I_{i n c}^{++}-I_{i n c}^{+-}}{I_{i n c}^{++}+I_{i n c}^{++}}
$$

where $I_{\text {inc }}$ are the average incoherent scattered intensities which are detected in the same $(\|)$ or orthogonal $(\perp)$ polarization states, respectively, relative to the incident light. Normally, a certain polarization state is destroyed exponentially, i.e. $\mathcal{P}(s) \propto \exp \left(-s / \ell_{p}\right)$ where $\ell_{p}$ is the characteristic average depolarization length. For Rayleigh scatterers, $\ell_{p}$ is of the order of $\ell^{\star}$ and becomes a few $\ell^{\star}$ for large Rayleigh-Gans-Debye scatterers. $\mathcal{P}$ also depends on the geometry. Simulations show that for Rayleigh scattering, $\mathcal{P}_{c}$ is even slightly negative in backscattering geometry.

In the following we will derive a relationship between the coherent backscattering in the crossed polarized states $C B E^{\perp}$ at $q_{b}=0$ and the degree of polarization of the multiply scattered light. The complete curve shape of $C B E^{\perp}\left(\vec{q}_{b}\right)$ is difficult to derive analytically and we will restrict ourselves to simulations. It is rounded off very strongly as only some degree of polarization persists for the short paths. The long paths, which are responsible for the cusp of the cone, are completely decorrelated on the direct and reversed paths with respect to their polarization. In order to calculate the factor $C B E^{\perp}$, we have to evaluate in analogy with eq. (1.40) the expression:

$$
C B E^{\perp}=1+\frac{2 \Re\left[e_{\otimes}^{\perp} e_{\odot}^{\perp *}\right]}{\left|e_{\otimes}^{\perp}\right|^{2}+\left|e_{\odot}^{\perp}\right|^{2}} .
$$

\footnotetext{
${ }^{13}$ The perpendicular polarization state for the direct and reversed path is obtained by a rotation of 90 degree in direction of propagation, respectively.
} 
The result is compared to the amount of the remaining polarization $\mathcal{P}$ (after backscattering), which can be calculated by evaluating the terms $I_{i n c}^{\|}=\left|e_{\otimes}^{\|}\right|^{2}$ and $I_{\text {inc }}^{\perp}=\left|e_{\otimes}^{\perp}\right|^{2}$. It is important to note that in the local coordinate system of the light paths, in backscattering geometry, the laboratory frame switches from a right- to left-handed coordinate system. We take this rotation

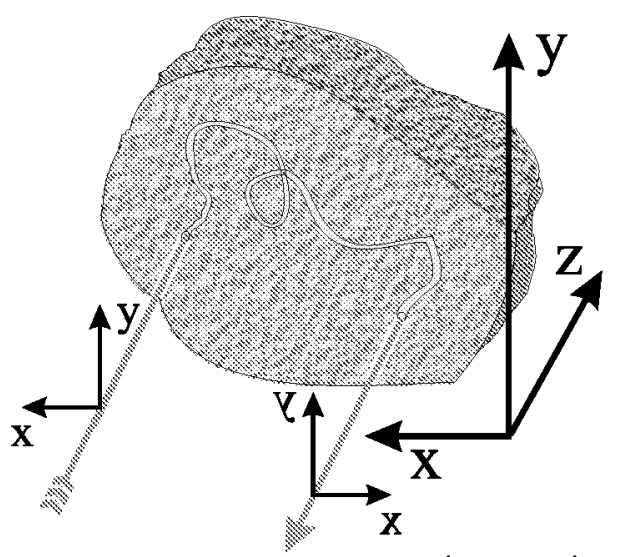
into account by multiplying $M$ from the left with the matrix $\mathbb{I}:=\left(\begin{array}{cc}-1 & 0 \\ 0 & 1\end{array}\right)$, corresponding to a reflection. With these notations one obtains for example:

$$
\begin{aligned}
I_{i n c}^{x x}=\left|e_{\otimes}^{x x}\right|^{2} & =\left|\left(\begin{array}{l}
1 \\
0
\end{array}\right)\left(\begin{array}{cc}
-1 & 0 \\
0 & 1
\end{array}\right)\left(\begin{array}{ll}
a & b \\
c & d
\end{array}\right)\left(\begin{array}{l}
1 \\
0
\end{array}\right)\right|^{2}=|a|^{2} \\
I_{i n c}^{x y}=\left|e_{\otimes}^{x y}\right|^{2} & =\left|\left(\begin{array}{l}
0 \\
1
\end{array}\right)\left(\begin{array}{cc}
-1 & 0 \\
0 & 1
\end{array}\right)\left(\begin{array}{ll}
a & b \\
c & d
\end{array}\right)\left(\begin{array}{l}
1 \\
0
\end{array}\right)\right|^{2}=|c|^{2} \\
\left|e_{\otimes}^{x y}+e_{\odot}^{x y}\right|^{2} & =\left|\left(\begin{array}{l}
0 \\
1
\end{array}\right) \amalg \mathrm{M}\left(\begin{array}{l}
1 \\
0
\end{array}\right)+\left(\begin{array}{l}
0 \\
1
\end{array}\right)(\mathbb{I M})^{\top}\left(\begin{array}{l}
1 \\
0
\end{array}\right)\right|^{2}=|b-c|^{2}
\end{aligned}
$$

If the sample is rotated by 90 degrees, the matrix $M$ which represents the light path within the sample, must be rotated by 90 degrees or, equivalently, the polarizer and analyzer must be rotated by -90 degrees. In the following, the different orientations of the sample are symbolized by $\square$ for the cases (1.54,1.55), $\square$ for a rotation by 90 degrees and for a rotation by 45 degrees. $\square$ symbolizes the average over $\square$ and $\square$, and $\varnothing$ the average over all positions, i.e. the sample is rotated during the measurement. In this way, one finds (see Appendix B) the following relations between the coherent backscattering cone in exact backscattering direction and the remaining degree of polarization in the crossed channels:

$$
\begin{gathered}
C B E_{q_{b}=0}^{+-}=1+\mathcal{C}_{o}^{ \pm}\left(\frac{2 \mathcal{P}_{l}(\bigotimes)}{1-\mathcal{P}_{c}}\right)_{m s}=1+\mathcal{C}_{o}^{ \pm}\left(\frac{I_{o} \mathcal{P}_{l}(\bigotimes)}{I_{\text {inc }}^{+-}}\right)_{m s} \\
C B E_{q_{b}=0}^{x y}(\square)=1+\left(\frac{\mathcal{P}_{c}+\mathcal{P}_{l}(\bigotimes)}{1-\mathcal{P}_{l}(\square)}\right)_{m s}=1+\left(\frac{\frac{1}{2} I_{o}\left[\mathcal{P}_{c}+\mathcal{P}_{l}(\bigotimes)\right]}{I_{\text {inc }}^{x y}(\square)}\right)_{m s} .
\end{gathered}
$$

$I_{o}$ is the total, incoherent backscattered intensity; ' $m s$ ' indicates that only multiple scattering light paths (in backscattering direction) are taken into consid- 
eration. Obviously, the circular polarization averages over all rotations of the sample. The parameter $\mathcal{C}_{o}^{ \pm}$, which is defined in analogy to eq. (1.41), reflects the amount of single scattering. The case $(x y)$ suppresses single scattering, normally.

Eqs. $(1.57,1.58)$ can be explained in words as following: The height of the coherent backscattering cone in the crossed polarized channels is given by the amount of light which is scattered into the opposite polarization state via a polarization conserving channel. 'Polarization conserving channels' are those light paths whose matrices $(\mathbb{M})$ have the same eigen-vectors for the direct and reversed paths. One can show that this is equivalent to the condition that the eigen-vectors are perpendicular. On the other hand this is equivalent to the condition that the matrix $(I M)$ is symmetric. As already mentioned above, if the matrix representing a certain light path is symmetric, the matrices of the direct and reversed paths are identical and $C B E$ equals two, independent of the polarization of the incident and detected light. If, however, the matrix is not completely symmetric, which is normally the case, it can be separated into a symmetric and an antisymmetric part $\mathbb{I} M=(\mathbb{I M})^{s}+(\mathbb{I M})^{a}$. With eq. (1.53) and the relations $\left[(\mathbb{I M})^{s}\right]^{\top}=(\mathbb{I M})^{s},\left[(\mathbb{I M})^{a}\right]^{\top}=-(\mathbb{I M})^{a}, e_{\otimes}^{\perp}=\vec{P}^{\perp} \mathrm{M} \vec{P}$ and $e_{\odot}^{\perp}=\vec{P} \mathrm{M}^{\top} \vec{P}^{\perp}$ one obtains:

$$
C B E^{\perp}=1+\mathcal{C}_{o}^{\perp} \frac{\left|\overrightarrow{P^{\perp}} \mathrm{M}^{s} \vec{P}\right|^{2}-\left|\overrightarrow{P^{\perp}} \mathrm{M}^{a} \vec{P}\right|^{2}}{\left|\vec{P} \perp \mathrm{M}^{s} \vec{P}\right|^{2}+\left|\vec{P} \perp \mathrm{M}^{a} \vec{P}\right|^{2}}
$$

Normally, the symmetry decreases with increasing path length. Therefore, only short paths contribute to coherent backscattering in the crossed channels, giving a (small) broad cone. An example for highly symmetric paths is shown in the fig. 1.15, assuming large (Mie) spheres which scatter light in a diagonal plane on a smooth curve. These light paths transfer light from one linear polarization state

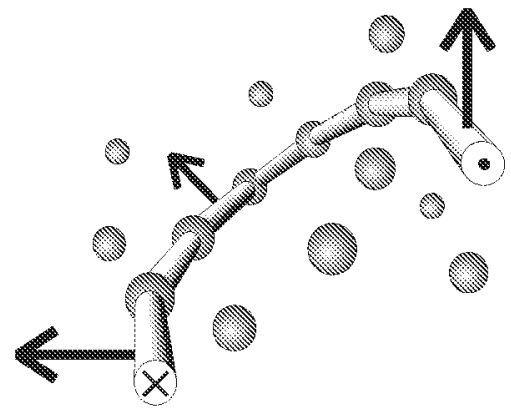

Figure 1.15 to the orthogonal one as the depolarization by the Mie spheres is relatively slow. Therefore, $C B E^{x y}$ for Mie spheres looks like a four-leafed clover (see fig. 1.21). The weight of such types of paths with respect to the total number of paths determines if a cone in the crossed channels is visible or not. 
If the sample is symmetric by rotation around the incident wave vector, eqs. $(1.57,1.58)$ reduce to

$$
\begin{aligned}
& C B E_{q_{b}=0}^{+-}=1+\mathcal{C}_{o}^{ \pm}\left(\frac{I_{o} \mathcal{P}_{l}}{I_{i n c}^{+-}}\right)_{m s} \\
& C B E_{q_{b}=0}^{x y}=1+\left(\frac{\frac{1}{2} I_{o}\left(\mathcal{P}_{c}+\mathcal{P}_{l}\right)}{I_{\text {inc }}^{x y}}\right)_{m s} .
\end{aligned}
$$

For non-polarized light one obtains $\overline{C B E}_{q_{b}=0}=1+\frac{I_{o}^{m s}}{I_{o}^{m s}+I_{o}^{s s}}\left(\frac{1}{2}\left(1+\mathcal{P}_{c}\right)+\mathcal{P}_{l}\right)_{m}$ which is the weighted average value of the cases ' ++ ' and ' +- ' or ' $x x$ ' and ' $x y$ '.

From these equations a relation between $\mathcal{P}_{l}$ and $\mathcal{P}_{c}$ can be obtained. Since $C B E^{+-}$and $C B E^{x y}$ must be in the interval $[0,2]$ and $\mathcal{P}_{l}$ and $\mathcal{P}_{c}$ in the interval $[-1,1]$, we obtain in the case of a rotational invariant sample in the backscattering direction (now single scattering is included as it can be formally added to $C B E)$ :

$$
\frac{\mathcal{P}_{c}-1}{2} \leq \mathcal{P}_{l} \leq \frac{1-\mathcal{P}_{c}}{2}
$$

This relation is shown in the figure to the right. The point , for example, corresponds to light paths which guide the light (as in an ideal monomodal fibre) without circular depolarization. A mirror is represented by the point $\boldsymbol{\nabla}$. The point $\mathbf{A}$ can be obtained with 3 mirrors as indicated in the figure, which corresponds to the matrix $M=\left(\begin{array}{cc}0 & -1 \\ -1 & 0\end{array}\right)$. As in this case $\mathcal{P}_{c}$ and $\mathcal{P}_{l}$ are negative, according to eq. (1.60), there would be a destructive interference in the case

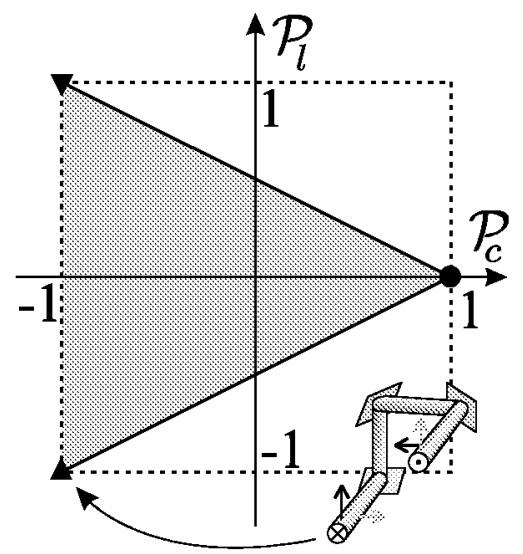
of $C B E^{\perp}$. Of course, this type of 'scatterers' is very unusual. However, such a destructive interference of $\mathrm{CB}$ was predicted for elastically scattered neutrons [45], when the neutrons are scattered into the orthogonal spin state.

The theorem of reciprocity is not restricted to paths in backscattering geometry. Thus, a relation for the polarization in transmission can be also obtained. In transmission geometry (denoted by ' $t$ '), the matrix $\mathbb{1}$ must be replaced by the identity. The vectors $\vec{P}, \vec{A}$ are the same when calculating the coherent backscattering enhancement but the complex conjugate when calculating $\mathcal{P}_{c}^{t}$ (due to the different position of the circular analyzer). Thus one 
obtains the relation:

$$
-\frac{1+\mathcal{P}_{c}^{t}}{2} \leq \mathcal{P}_{l}^{t} \leq \frac{1+\mathcal{P}_{c}^{t}}{2} .
$$

which corresponds to the relation in backscattering geometry but reflected at the $\mathcal{P}_{l}$-axis.

Fig. 1.16 shows the coherent backscattering enhancement for Rayleigh and Mie-scattering, for the linear parallel $(x x)$, linear crossed $(x y)$, circular $(++)$ and circular crossed (+-) polarization states, as well as for non-polarized light. These data were calculated using radiative transfer theory and verified by numerical simulations. Especially for Rayleigh scattering $(k a \rightarrow 0), C B E^{x x}$ has a value of 1.7521 (due to single scattering), i.e. smaller than the theoretical possible value of two. With absorption, the relative importance of single scattering would increase and $C B E^{x x}$ would still decrease. The larger the particles, the more the scattering is anisotropic and forward directed. Consequently, the amount of single scattering decreases with increasing $k a$. In the case of circular polarization $(++)$, single scattering is suppressed as it is comparable to a reflection in a mirror, which flips one circular polarization state into the other. Consequently, $C B E^{++}$is always two, independent of $k a$ and absorption. In the case of non-polarized incident and/or detected light, $C B E$ is a few per cent larger than the average value, due to the fact, that the cases $C B E^{x x}, C B E^{++}$, $C B E^{x y}$ and $C B E^{+-}$are weighted differently, according to their different amount of incoherent intensities.

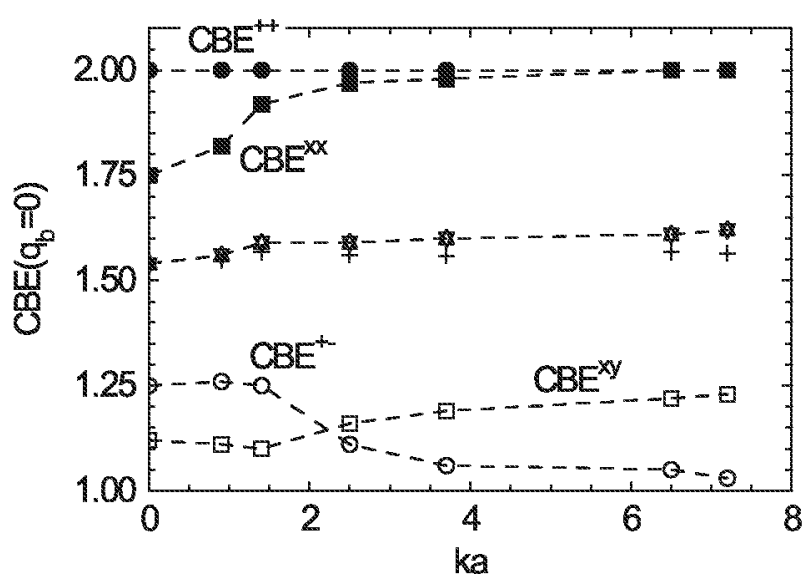

Figure 1.16: Coherent backscattering in the exact backscattering direction as a function of the size parameter $k a$ ( $k$ wave vector, a radius of scatterers), for the different polarization states: same circular incident as detected polarization; linear parallel; $\bigcirc \square$ orthogonal incident and detected polarization; + average value of the four preceding curves; unpolarized light. Relative index of refraction scatterers/surrounding media $m=1.2$. From [47, 71]. 


\subsubsection{Monte-Carlo simulations}

Within the diffusion approximation one cannot obtain a satisfying analytic expression for the path length distribution for short paths. However, it is possible to simulate most multiple scattering problems, especially coherent backscattering within a short time (minutes to some hours) on a PC and incorporate surface effects, anisotropic scattering and polarization. The basis of such simulations is the random walk model where light paths are randomly generated as follows: A new direction after a scattering event is chosen according to the probability, given by the differential cross section for the intensity $d \sigma(\theta, \phi)$ that the light is scattered in a certain direction. The distance $\Delta r$ between two scattering events is chosen according to the Beer-Lambert law by $p(\Delta r)=\exp (-\Delta r / \ell) / \ell$. The absolute intensity is renormalized to unity at each step, i.e. we consider a random walk of photons. Eventually, the light is absorbed with a probability proportional to $\exp \left(-\Delta r / \ell_{a}\right)$ or, equivalently, a certain maximum path length $s_{\max }$ is chosen for each photon according to a distribution $\exp \left(-s_{\max } / \ell_{a}\right)$. A photon can be reflected at the surface or a window or leave the sample before being absorbed. The positions of the scatterers are not monitored corresponding to an incoherent summation of all light paths. At the end, the number of photons which have left the sample in transmission or reflection is counted as a function of position, path length, phase and polarization.

In principle, with this random walk model of particles it is impossible to obtain interference effects between different paths. However, CB is just the interference between each light path and its reversed path which, moreover, are related by the theorem of reciprocity. Thus, the shape of the CB-cone is essentially the Fourier transform of the intensity distribution $I(\vec{r})$ which can be obtained by the random walk model described above. Therefore, the CB-cone can be completely simulated by this model, polarization effects included.

There is a very efficient modification of this random walk model which is called the method of 'partial photons' [46] (for more details see Appendix C). This technique is very well suited to the problem of coherent backscattering. At each scattering event, the probability is calculated that the light leaves the sample in the exact backscattering direction. This probability is given by the probability that the light is scattered in this direction, times the probability for leaving the sample without being scattered again, times the probability for not being absorbed. The first probability is given by the differential cross section, that the light is scattered in this direction. The second and third 
probabilities are given by $\exp (-z / \ell) / \ell$ and $\exp \left(-z / \ell_{a}\right) / \ell_{a}, z$ being the distance of the scattering event from the surface. Now, instead of counting the number of photons which have left the sample, these probabilities (at each scattering event) for leaving the sample are summed. Each light path contributes with its 'partial photons' to the summation until it is absorbed or until it has left the sample. The result is essentially the same as compared to the 'classical' method. Strictly speaking, it is even more correct as only light which leaves the sample in a very small angular range (as it is the case for CB) is considered. In any case, the method of partial photons is much faster than the 'classical' method. 


\subsubsection{Experimental setup of CB}

The picture in fig. 1.17 shows an experimental setup used to measure coherent backscattering. The light source is a continuous wave $\mathrm{Ar}^{+}$-laser with an output power of up to 0.5 Watt. Less laser power can be used for samples with higher reflectivity or when using a camera with higher sensitivity or less angular resolution. The coherence length of the laser is not important (see also section 1.3.4 and fig. 1.24) while the spatial coherence and homogeneity of the wave front are essential. The intensity of the laser beam is controlled by a rotatable $\boldsymbol{\lambda} / 2$-plate and a linear polarizer. This provides a better pointing and divergence stability as compared to changing the laser power directly. A second $\lambda / 2$-plate is used to rotate the polarization by $90^{\circ}$ in order to detect the crossed polarization states. The beam expander, necessary to create a largely extended beam in order to approach a plane wave, is composed of a microscope objective (focusing the laser beam on a pin-hole) and a second lens in a telescopic configuration. The laser beam is typically expanded to a diameter of about $15 \mathrm{~mm}$. The semi-transparent mirror is the most important element in the setup since it creates most of the experimental artifacts. Only in the case of very strongly scattering samples with rather large cones the semi-transparent mirror can be replaced by a small conventional mirror without too much shadowing of the cone tip. The semi-transparent mirror should be wedge shaped and relatively thick in order to reduce interference fringes (due to reflections from the mirrors front and back face). It is advantageous to choose the (linear) polarization of the incident laser beam such that the (Rayleigh) scattering from defects of this mirror has a minimum in the direction of the camera. The part of the incident laser light which is deflected by the semi-transparent mirror must be completely absorbed. We use for this purpose a black filter oriented at the Brewster angle or a second filter to eliminate the reflection coming from the first filter. With the help of an additional $\boldsymbol{\lambda} / 4$-plate, it is possible to measure the cone in the circular polarized states. By using a single $\lambda / 4$-plate at this position instead of one in front of the semitransparent mirror and another in front of the analyzer, one also circumvents the problem that the reflectivity of

Figure 1.17: Photo of an experimental setup used to measure coherent backscattering with a CCD-camera (see text).

next page $\rightarrow$ 


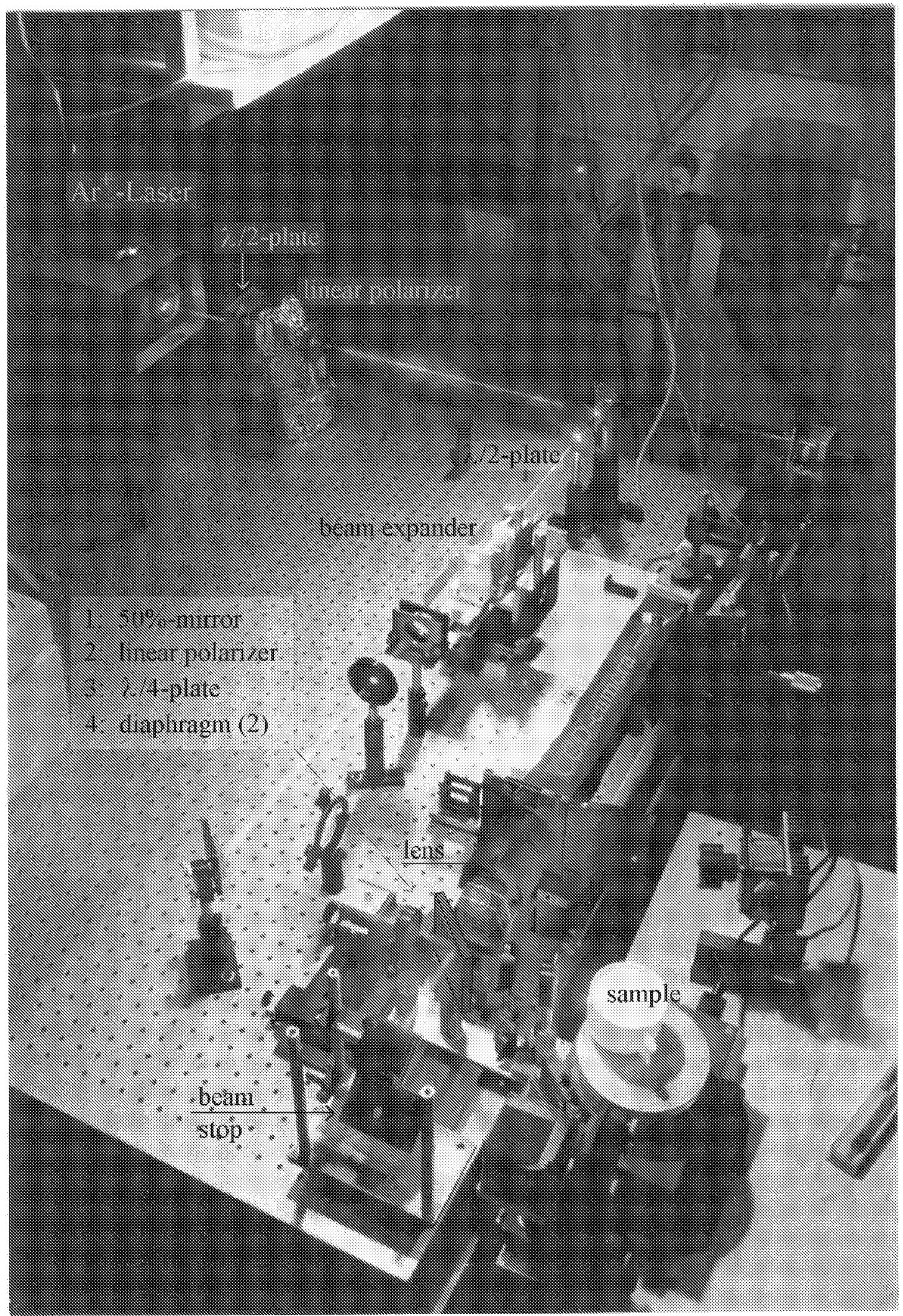


a semi-transparent mirror is somewhat-polarization dependent. A single $\lambda / 4$ plate is sufficient as in the backscattering direction the local coordinate system is rotated by $180^{\circ}$. The cases $C B E^{++}$and $C B E^{+-}$are chosen by the corresponding position (parallel or perpendicular) of the linear analyzer. The light which is scattered back from the sample is half-deflected by the semi-transparent mirror, passes through a linear polarizer cube and is focused by a lens on the CCD-camera situated in the focal plane. The camera, which must have a linear response, records a two dimensional image of the intensity as a function of the scattering angle around exact backscattering. Special care must be taken in order to ensure measurement of the absolute intensity. To this end a dark reference must be subtracted from the sample measurement and the different angular regions on the camera must be calibrated by a reference measurement which shows no coherent backscattering. The thickness of the sample and the diameter of the illuminated region should be larger than about $10 \ell^{\star}$. If the sample is not liquid, the speckle pattern must be averaged out, for example by rotating the sample. The most effective averaging is obtained by wiggling and rotating the sample at the same time. ${ }^{14}$ It is also possible to keep the sample fixed but illuminating it via a wiggling mirror. This procedure does not change the position of the CB-cone as both the direct and reversed paths to and from the sample experience the same changes. In fact, for this reason any optical element between semi-transparent mirror and sample is less critical with respect to optical quality (besides direct reflections by defects) and translation during the measurement. To some extent coherent backscattering acts like a phase-conjugating mirror which corrects for defects in the light path.

If the sample is located behind a window, one has to correct for the (multiple) reflections in the window [48]. These may change $p(s)$ substantially. This effect may be reduced by using a thicker window or index matching. On the other hand, one can try to include these effects into simulations or theory. The position of the sample is of no importance. It can be placed up to several focal length (of the camera lens) in front of the semi-transparent mirror. This is a significant advantage of coherent backscattering when used as a remote sensing technique.

Instead of the camera, a (monomodal) fibre combined with a photomultiplier, mounted on a rotational stage, can be used. ${ }^{15}$ This provides a higher

\footnotetext{
${ }^{14}$ Just translating the sample is less effective, as completely independent speckle patterns are only obtained for translations larger than the beam diameter.

${ }^{15}$ An additional improvement provides an 'off-centre rotational stage' [49], where the
} 
dynamic range and, more important, a larger angular resolution compared to a standard CCD-camera with $512 \times 512$ pixels and 256 grey levels. For Rayleigh scattering, for example, the shape of the CB-cone decreases relatively fast around the maximum but very slowly at the wings. Thus, for very precise and complete cone shape measurements it becomes necessary to translate the camera or to vary the angular resolution. Nevertheless, only with a camera relatively fast (in-line) measurements and two-dimensional images in 'one shot' are possible.

The position of the camera, as well as the divergence of the expanded laser beam, are adjusted by using the fact that, to some extent, coherent backscattering acts like a time-reversal mirror (see Appendix D). Two measurements are necessary: 1) Measuring the coherent backscattering cone and selecting the position of the camera where $C B E$ is maximum. 2) Replacing the sample by a mirror and selecting the focal point, again by varying the position of the camera. If the incident wave is plane parallel on the surface of sample, then the positions of measurement 1) and 2) will be identical. However, if the laser beam is divergent, position 1 is shifted forward and position 2 backward indicating misalignment of the beam expander.

In our experience, by using a fixed camera, values of $\ell^{\star} \gtrsim 10 \mu \mathrm{m}$ can be measured (for visible light). By using a rotational stage there is no lower limit for $\ell^{\star}$. It is difficult to estimate the upper detection limit for $\ell^{\star}$. The diffraction limit of a laser beam which has a homogeneous intensity distribution over a diameter $d$ amounts to $\lambda / d \approx 50 \mu r a d$. Consequently, with a typical laser beam diameter of about $1 \mathrm{~cm}$, only values $\ell^{\star} \lesssim 200 \mu \mathrm{m}$ can be measured. In reality, however, the intensity profile of the reflecting area on the sample is more Gaussian-like and the resolution much better. But a Gaussian intensity profile reduces the enhancement factor because in this case the intensity at the beginning and the end point of a random walk may no longer be identical. Normally, we cut off the beam profile with a diaphragm

whole setup (besides the incoming laser-beam) is rotated about the image point of the sample with respect to the semi-transparent mirror. At the same time the sample is rotated in the opposite direction. With this setup the direction and position of the incident light with respect to the sample is always the same. Also the light path to the detector is not changed. Consequently, the angle of observation is changed without changing the angle of the light paths with respect to the different optical elements. Only the angle between the incident laser beam and the semi-transparent mirror changes. However, the corresponding change in the reflectivity can be measured and corrected for easily with a detector at the place of the sample. 
in order to obtain a more or less homogeneous intensity distribution on the sample, but this is always a compromise. If possible, another diaphragm is placed directly in front of the sample in order to block light coming back from the outside of the illuminated region. These light paths would not be followed in the opposite direction and thus reduce CB. For larger values of $\ell^{\star}$ a good solution is to use divergent light (see Appendix D). Then, the only limiting factors are the sample size and the sensitivity of the system, where the stray light from the semi-transparent mirror and the $\lambda / 4$-plate plays an important role.

A completely different technique is 'heterodyne detection' [50] which, however, has only been used for very special 'samples' composed of a phase screen in front of a mirror. 
Figure 1.18: Coherent backscattering cones for different materials. Circular polarization, $\lambda=0.514 \mu m$. For comparison, the coherent parts are rescaled to obtain the theoretical enhancement factor of two. Except for styropor and snow where the limited angular resolution of the used setup was respected. The measured tip

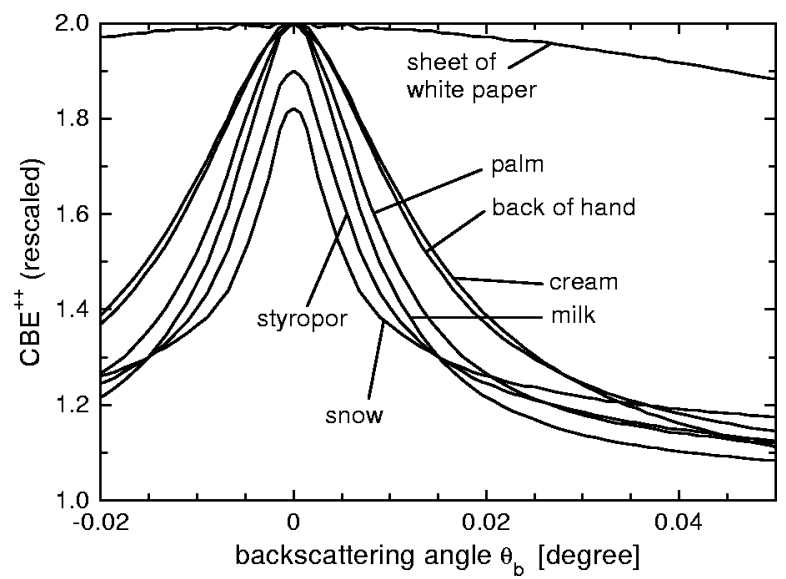
values lie between 1.6 and 1.95 .

\subsubsection{Examples of CB-cones}

In this section, some examples of experimental CB-cones will be discussed with emphasis on the cone shape. As discussed in section 1.3.1, the slope near the top of the cone is determined by the long light paths and, consequently, depends only on quantities such as the transport mean free path $\ell^{\star}$, the absorption length $\ell_{a}$ and the boundary conditions describing diffuse light transport. The wings of the cone, however, depend in addition on the size of the scatterers and on the polarization. Fig. 1.18 shows CB-cones of different materials with a cone width varying from about 0.01 to 0.4 degree. This corresponds to values of $\ell^{\star}$ between $0.4 \mathrm{~mm}$ for snow and $10 \mu \mathrm{m}$ for paper. Most of these cones are rounded off due to absorption or, in the case of paper, due to a limited sample thickness. Note that cutting off of the long paths by absorption or a limited sample thickness does not destroy the enhancement factor of two. Fig. 1.19 shows a very precise measurement from a powder of $\mathrm{TiO}_{2}$ particles. This cone is much wider because of a transport mean free path below $1 \mu \mathrm{m}$. In this case, the cone shape fits very well to the theory of Akkermans et al. $[43,44]$. Fig. 1.20 shows the CB-cones in the case of Rayleigh scattering for the different polarization states. The sample is a suspension of colloidal polystyrene spheres with a diameter of $100 \mathrm{~nm}$ in water. From Mie-theory one obtains in this case a ratio $\ell^{\star} / \ell=1.13$, quite close to Rayleigh scattering. For circular polarization the cone has a cylindrical symmetry and a small cone is found even in the helicity non-preserving channel $(+-)$. The measurements agree very well with simulations but not with eq. (1.46). The wings of $C B E^{+-}$ are higher compared to those of $C B E^{++}$due to the fact that, on the short 


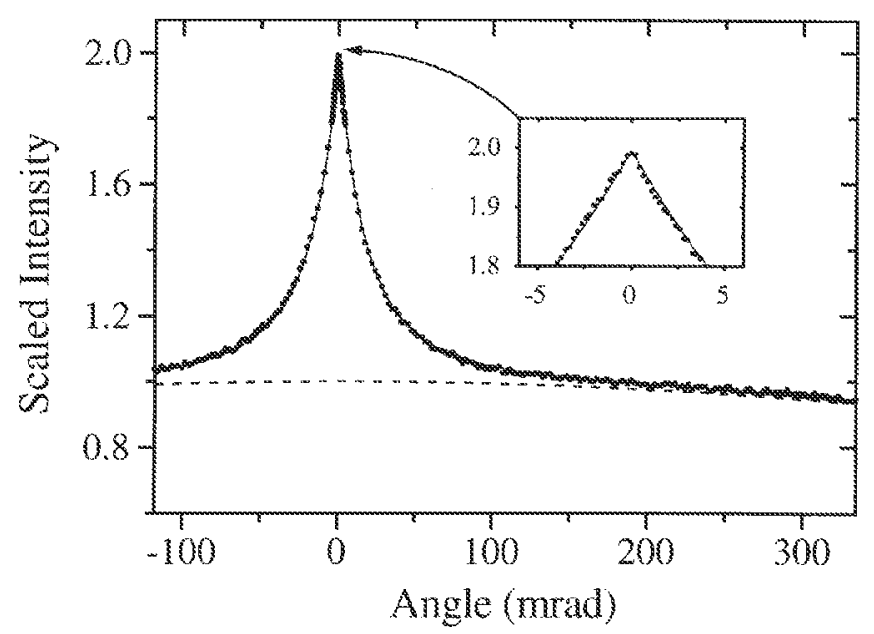

Figure 1.19: Coherent backscattering cone of a white powder of $\mathrm{TiO}_{2}$ with essentially no $a b$ sorption. Measured by Wiersma et al. [49] for circular polarization and $\lambda=0.514 \mu \mathrm{m}$. The triangular shape and an enhancement factor very close to two are clearly visible.
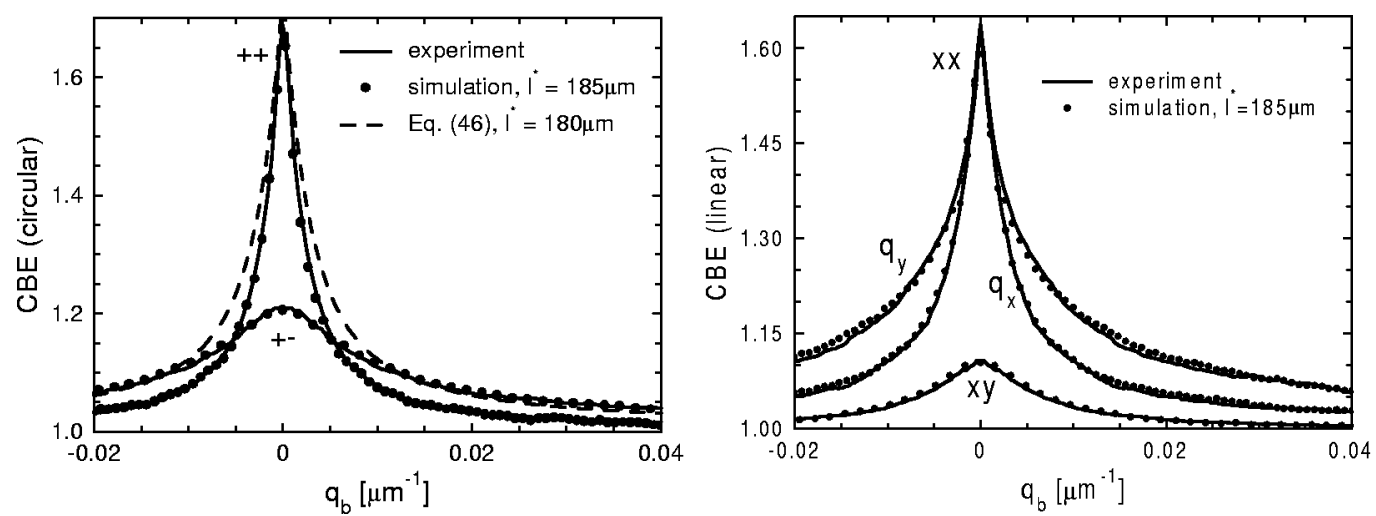

Figure 1.20: CB-cones of a sample of colloidal polystyrene spheres with a diameter of $100 \mathrm{~nm}$ in water for various polarizations of incident and detected light; $\lambda=0.5145 \mu \mathrm{m}$, volume fraction $2.9 \%$, refraction index ratio polystyrene/water $=1.19$. Comparison with simulations gives a value of $\ell^{\star}=185 \mu \mathrm{m}$, compared to a value of $175 \mu \mathrm{m}$ obtained from Mie-theory. The incident light is polarized in the $y$-direction.

paths, more light is scattered in the opposite than in the same circular polarization state. In fact, in the case of Rayleigh scattering the degree of remaining circular polarization $\mathcal{P}_{c}$ is slightly negative. In the case of linear polarization, the cone shape is elliptical. This asymmetry is a consequence of the doughnutlike form factor of Rayleigh scattering which results in anisotropic low order multiple scattering clearly visible in the wings. In contrast, the central part is isotropic indicating the isotropy of high order scattering.

Fig. 1.21 shows the coherent backscattering cones of relatively large oil 
Figure 1.21: $\quad$ Two $d i$ mensional, angle-resolved intensity plot of a 20/80 vol. fraction oil/water emulsion (average droplet size $\approx$ $7 \mu \mathrm{m}$ ) for different polarization states. Angular range $0.27 \times 0.24$ degrees. For better visibility, the contrast of the pictures is enhanced. In reality, we measured for the maxima: $C B E^{++}=C B E^{x x}=$ $1.8, C B E^{+-}=1.1$ and $C B E^{x y}$ $=1.3$.

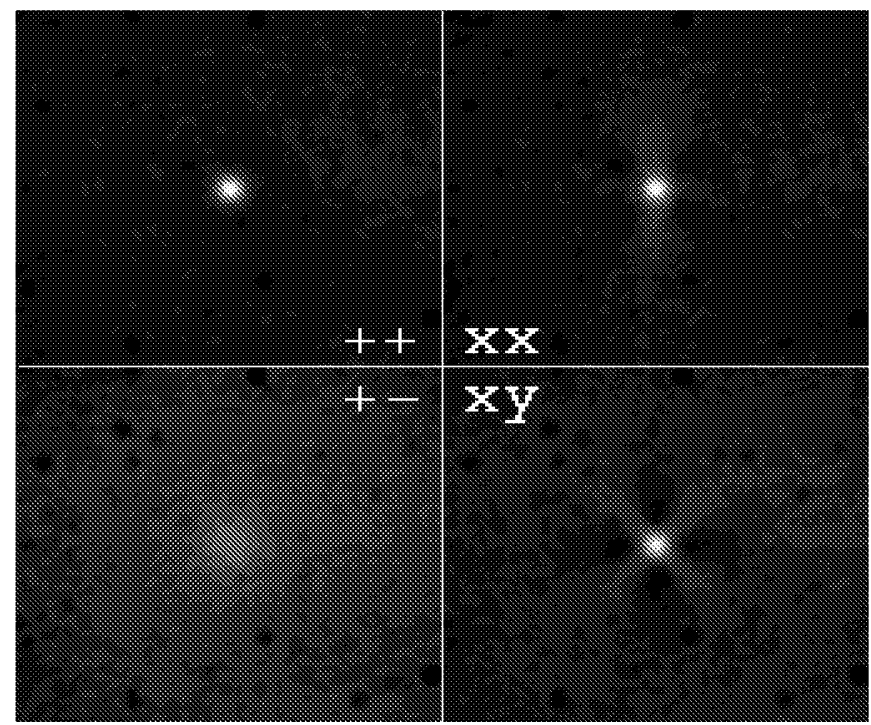

droplets in water corresponding to very large Mie-spheres. While $(++)$ and $(+-)$ are isotropic, $(x x)$ and $(x y)$ show a two- and four-fold symmetry. The four-fold symmetry in the case $(x y)$ corresponds to paths which are illustrated in fig. 1.15. Along this direction, light is scattered into the orthogonal polarization states very efficiently. The dark regions in-between (they are even darker than the average intensity at large angles) indicate that less light is scattered into the orthogonal polarization states along this direction. In other words, the scattering does not only depend on the length of a path $s$ but on its configuration as well. The radial intensity distribution $I(\vec{r})^{x y}$ around an illuminated spot is no longer rotational invariant for this sample. Consequently, $C B E^{x y}\left(\vec{q}_{b}\right)$, which is the Fourier transform of $I(\vec{r})^{x y}$, is also not rotational invariant. Obviously the weighting of this type of paths is not negligible in the case of large Mie-spheres where $\ell^{\star} / \ell \gg 1$.

Fig. 1.22 shows the coherent backscattering cone of a stretched plexiglass sample which becomes strongly turbid under mechanical deformation. In this case, surprisingly the cone becomes asymmetric even in the case $C B E^{++}$. This is because $\ell^{\star}$ becomes different in the $x$ - and $y$-direction due to structural inhomogeneities which orient under deformation. The asymmetry is most pronounced when the PMMA just becomes turbid. With increasing deformation, these inhomogeneities progressively overlap with the result that the overall anisotropy of the stretched PMMA glass decreases again.

Coherent backscattering of light has been studied over recent years on many types of strongly scattering complex materials. Examples range 


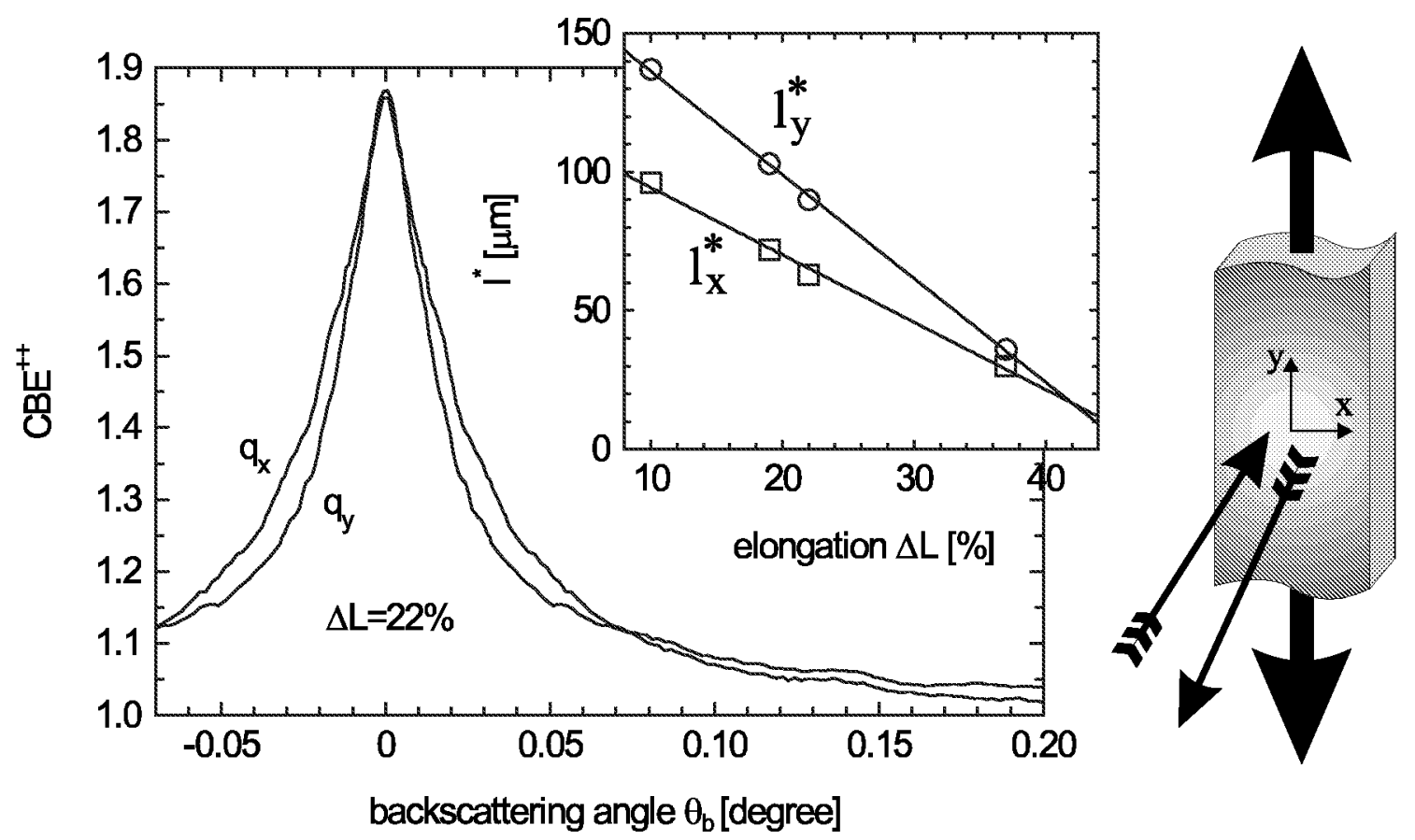

Figure 1.22: Coherent backscattering cone of stretched plexiglass [51]. Circular polarization, $\lambda=0.5145 \mu \mathrm{m}$. In this case the optical density, i.e. $\ell^{\star}$ is anisotropic. The inset shows the anisotropy of $\ell^{\star}$ in the $x$ - and $y$-directions as a function of the deformation. Surprisingly, with increasing deformation, the anisotropy becomes smaller.

from liquid crystals [52], fractals [44], [54]-[56] to rough surfaces [57, 58]. In addition, the influence of a non-linear part in the optical polarizability has been studied on coherent backscattering [53]. In optically amplifying materials, the intensity increases with path length and consequently, the cone shape becomes narrower with increasing gain [59,60]. This effect can be described in analogy to absorption but with a 'negative' absorption length. Therefore, in analogy to absorption, the enhancement factor is not changed in a medium with gain. Moreover, time dependent measurements of the CB-cone have been performed [61] and the influence of the surface was investigated to some extent [48, 62]-[64]. Apart from light waves, the CB-cone has also been measured for acoustic waves $[65,66]$.

The only effects which possibly destroy optical coherent backscattering are i) inelastic scattering by relativistically moving scatterers [36] or fluorescence and ii) magneto-optical Faraday rotation $[36,37]$. Faraday rotation (FR) oc- 
curs in case of a (slightly) different index of refraction for the two circular polarization states of the light which propagates parallel to a magnetic field. Moreover, the sign of the rotation changes with the direction of propagation relative to the magnetic field. These opposite phase shifts, $\Delta \alpha=V \vec{B} \overrightarrow{\Delta r}$ for the circular polarization states, provide a rotation of the linear polarization. $V$ is the material-specific Verdet constant, $\vec{B}$ the external magnetic field and $\overrightarrow{\Delta r}$ the propagated distance. FR breaks the theorem of reciprocity since, due to the external magnetic field, the symmetry between the direct and the reversed path is broken. In fact, the matrix which describes FR is the same for the direct and the reversed path and not the transposed one. Consequently, the matrix $M$ describing a total scattering path, FR included, is a product of matrices which are the transposed and matrices which are the same for the direct and the reversed path. Hence $M_{\odot} \neq M_{\otimes}^{\top}$, in general. The influence of FR on CB can be approximately described as follows: Between two scattering events, the field-induced phase difference between the direct and the reversed path segment is $2 \Delta \alpha$. Consequently, the contribution of this segment to the coherent backscattering enhancement becomes only $1+\cos (2 \Delta \alpha)$ and not 2 . In a simple model one may assume that these phase shifts are independent from segment to segment. Consequently, on average over all configurations and orientations, the total decrease in coherent backscattering for a path of length $s$ becomes: $C B E(V B, \diamond)=1+\langle\cos (2 \Delta \alpha)\rangle^{\wedge / \ell^{\star}} \approx 1+\exp \left[-\frac{1}{3} \curvearrowright \ell^{\star}(2 V B)^{2}\right]$, where the second term holds for small values of $(V B)^{2}$. In analogy to the cut-off scattering vector $q_{a}=\sqrt{3 / \ell^{\star} \ell_{a}}$ in the case of absorption (see eqs. 1.44 to 1.50), this corresponds to a cutoff vector $\vec{q}_{F R}=2 V \vec{B}$ for FR. Thus, like in the case of absorption, the cone is rounded off according to the substitution $q_{b}^{2} \rightarrow q_{b}^{2}+q_{F R}^{2}$. However, unlike for absorption, the coherent backscattering enhancement decreases because the non-coherent intensity is not changed by FR. Therefore, the influence of FR on coherent backscattering is given by the un-normalized curves in fig. 1.13. This model has been verified experimentally [38]-[40] for samples which are well described by the diffusion approximation. However, the simplifications made above, concerning the independence of the FR on each scattering event, are not always correct. In particular, if a single scattering event does not completely depolarize the light, or - equivalently - if there is coherent backscattering in the crossed channels, more subtle effects appear (see fig. 1.23). With increasing magnetic field, the peak height of the CB-cone is decreased but also shifted away from the exact backscattering direction. This shift can be explained by short light paths for which the light is only weakly 


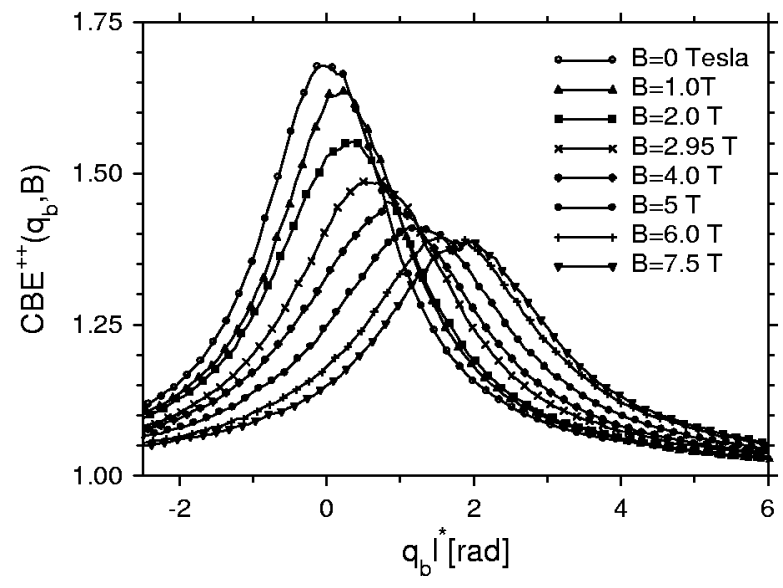

Figure 1.23 : Influence of Faraday rotation on coherent backscattering $\left(\mathrm{CBE}^{++}\right)$for a sample containing large Mie-scatterers, and using a magnetic field oriented perpendicular to the incident light. Sample: Faraday rotating glass containing $5 \%$ of air bubbles. Circular polarization, $\lambda=0.4579 \mu m$ [68].

depolarized. For these light paths the FR-induced phase shift and the phase shift corresponding to a direction of observation $\theta_{b} \neq 0$ are correlated. Thus both cancel at a field-dependent value $\theta_{b} \neq 0$ giving rise to a maximum of the $C B E$ at that particular angle off exact backscattering. In other words, for the short paths the backscattering vector must rather be replaced by $\vec{q}_{b} \rightarrow \vec{q}_{b}-\vec{q}_{F R}$ and not by $q_{b}^{2} \rightarrow q_{b}^{2}+q_{F R}^{2}$. But also for the long paths, i.e. in the diffusion limit, the simple model proposed above is not quite correct due to correlations between the influence of FR on CB and the scattering. For Rayleigh scattering we find that, due to this correlations, the influence of FR is two times higher than expected, i.e. $q_{F R}^{2}=2(2 V B)^{2}[67]$.

Can coherent backscattering be seen directly in nature? Can CB be seen with sunlight? It is interesting to note that the short coherence length of the sunlight (given by $\bar{\lambda}^{2} / \Delta \lambda \approx 1.5 \mu m$ ) does not affect $\mathrm{CB}$, as near the exact backscattering direction, the direct and reversed paths have essentially the same length. In fact, the short coherence length of the sunlight only results in a convolution of wavelength-dependent cones where the wavelength-range is determined by the detector. Consequently, the enhancement factor is not influenced by the short coherence length, only the cone shape changes. More crucial for $\mathrm{CB}$ is the size of the coherence area of sunlight as the starting and end points of the multiple scattered light paths must lie within one coherence area [69]. According to the discussion in section 1.2.2 on the speckle spot size, one can estimate that the coherence of sunlight on earth decreases to $50 \%$ within a diameter of $40 \mu \mathrm{m}$. Consequently, CB can be observed with sunlight for samples where the light paths essentially leave the sample within this distance, i.e. $\ell^{\star} \lesssim 40 \mu \mathrm{m}$. The data in fig. 1.24 are obtained for a sample of $\mathrm{BaSO}_{4}$-powder, measured - as usual - with an expanded, parallel laser beam and - in comparison - with sunlight. This sample has a transport mean free 


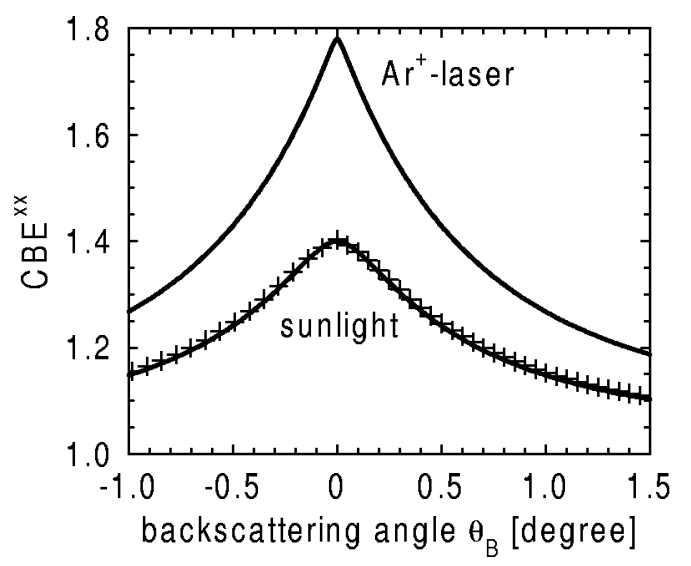

Figure 1.24: Solid lines: $C B-$ cone measured with Ar ${ }^{+}$-laser $(\lambda=$ $0.5145 \mu \mathrm{m}$ ) and sunlight [76]. In both cases the light was linearly polarized. The crosses represent a (rescaled) convolution of the laser cone with the divergence of the sunlight.

path of $\ell^{\star} \approx 5 \mu \mathrm{m}$ and consequently coherent backscattering is also observable with sunlight. However, the 'sunlight-cone' is smaller than two and rounded off as the long paths (which essentially contribute to the top) with starting and end points belonging to different coherence areas do not contribute to CB. Another, equivalent point of view is, that the 'sunlight-cone' is a superposition of several cones from independent sources which are spread in $\pm \theta_{b}$ over an angular range corresponding to the divergence of the sunlight. Consequently, the 'sunlight-cone' is obtained by a convolution of the 'laser-cone' with a function which reflects the angular-dependent intensity distribution of the sunlight. The crosses in fig. 1.24 are a (rescaled) convolution of the measured laser-cone with the Gaussian $\exp \left[-\left(\theta_{b} / 0.15\right)^{2}\right]$ corresponding roughly to the divergence of the sunlight. ${ }^{16}$ Consequently coherent backscattering can be seen with sunlight, if $\ell^{\star}$ is small enough. For objects which are further away from the sun, the divergence of the sunlight is smaller and consequently $\ell^{\star}$ can be correspondingly larger. In fact, an increased backscattering intensity is observed on Saturn's rings when sun, earth and Saturn are aligned. It can be attributed to coherent backscattering as it is created by the sunlight backscattered from small ice crystals [70]-[72]. The moons of planets which are further away from the sun than the earth show coherent backscattering as well. Also the earths moon has an increased surface brightness at full moon [73]. However, so far, it is not clear whether this effect of the earth moon must be attributed to coherent backscattering or to the so-called corn-field effect. The corn-field effect originates from the fact that in exact backscattering direction

\footnotetext{
${ }^{16}$ However, the convolution had to be rescaled in the height of the coherent part by a factor 0.57. Obviously, due to the difficulties in measuring the sunlight cone (large amount of diffuse light for example), it is much lower than expected.
} 

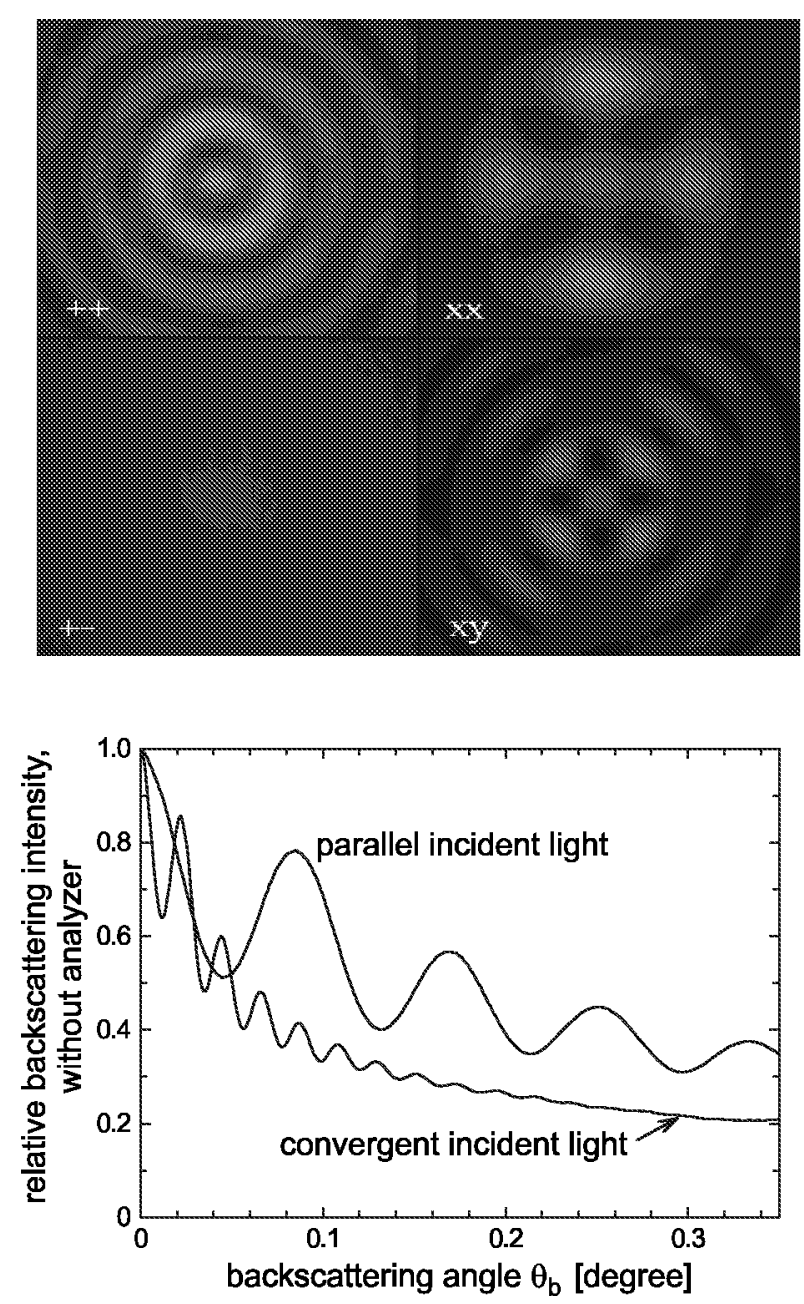

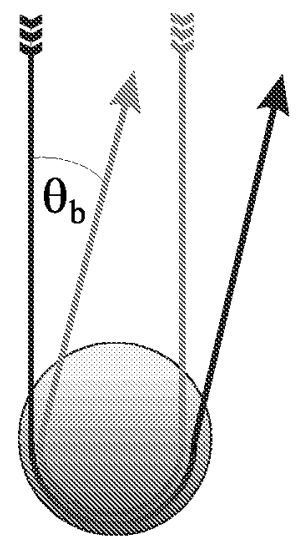

Figure 1.25:

Coherent backscattering of glass spheres. Diameter 0.355-0.4mm, volume fraction $<1 \%, \lambda=$ $0.5145 \mu \mathrm{m}$. The lower graph shows the angular intensity distribution for unpolarized light, with parallel and with convergent incidence.

no shadows are visible even on very rough surfaces. It can be seen, for example when looking at a corn-field, around the observers shadow at sun rise or sun set. This effect depends strongly on the geometry of the surface. Similarly one can observe enhanced backscattering around one's own shadow from sunlight falling on clouds or fog. This so-called Glory effect $[17,18]$ is mostly known from observation of the shadow of an airplane on a cloud. Fig. 1.25 shows backscattering measurements of a model system consisting of submillimeter size glass beads. As seen in the upper graph, the two dimensional intensity distribution around the exact backscattering direction strongly depends on polarization. $C B E^{x x}$ and $C B E^{x y}$ are not rotationally symmetric. In analogy to figs. 1.15 and 1.21 , this can be explained by the rotation of the polarization of the light which travels in the sphere in a diagonal plane. The lower graph shows the relative intensity distribution of non-polarized light as a function of 
the backscattering angle $\theta_{b}$. A series of oscillations can be seen. Of course, the non-polarized case, which was measured with parallel and convergent light, is rotationally invariant around exact backscattering direction. This scattering pattern must be an interference phenomena as the width of the oscillations is proportional to the wavelength and inversely proportional to the diameter of the spheres. Although it is tempting to utilize paths such as sketched in the fig. 1.25, the exact trajectories of light paths which contribute to the Glory are more complicated and depend on the index of refraction, the polarization of the incident and detected light, bead size and perfectness of the spheres. The curves which were obtained with parallel and convergent light are identical apart from a scaling factor which depends on the divergence of the incident light. This behavior is typical for CB and will be discussed Appendix D. Due to the wavelength dependence of the width of the oscillations, the Glory effect in nature shows coloured rings.

At the end of the preceding passage we tacitly made the transition from 'real' multiple scattering to 'multiple'-scattering in single, large particles. The angular dependence of the scattered intensity of such particles is given by their form factor to which however, in backscattering direction, CB always contributes to some extent. In the case of Figure 25, the glass spheres have not been perfectly round such that the backscattering pattern can really be attributed solely to the interference between the light paths in the spheres and their reversed paths, i.e. to CB. The Glory effect in nature is caused by perfectly round water droplets such that not only a direct and its reversed light path interfere but all light paths which are rotational symmetric around $\vec{k}_{i n}$.

Obviously, in the general case, there is a continuous transition from single to multiple scattering and often it is only a question of definition. It is important to note that if, by definition, single scattering of large particles is not included into $\mathrm{CB}$, the values of $\mathcal{C}_{\boldsymbol{o}}^{++}$and $\mathcal{C}_{\boldsymbol{o}}^{x y}$ (in analogy to the definition in eq. (41)) may also decrease below 1, because light may be backscattered into these polarization states already by one single particle (as can be seen for example in Figure 25). In many cases, single backscattering of larger particles gives a non-negligible contribution to the coherent backscattering cone. In these cases the shape of the cone can be considered as a superposition of two cones, one, which corresponds to the multiple scattering transport mean free path $\ell^{\star}$ and another, much broader, corresponding to the average size of the large, single scatterers. One example is the cone for snow in fig. 1.18, which has wings much 
higher than the other cones because of the single backscattering contribution from individual snow flakes. 


\subsection{Appendix A: Transport mean free path $\ell^{\star}$}

In this appendix we will discuss the length $\ell^{\star}$ more precisely. In fact, there are two definitions in literature for this characteristic length of a random walk. As long as the step length distribution of the scattering mean free path $\ell$ is exponential (Beer-Lambert), both definitions give the same result. However, for polymers (which can also be treated by a random walk model), where the segment length distribution is not exponential, the definitions give different results. Also Monte-Carlo simulations of light scattering, where the step length is chosen to be constant, do not give the correct results. The correct definition of the transport mean free path $\ell^{\star}$ is given via the mean square displacement as described in section 1.1. Another length in a random walk is the distance after which the information of the initial direction is lost, which corresponds to the distance after which the propagation is no longer ballistic and completely diffusive. This ballistic distance $l^{*}$ is obtained by evaluating the sum:

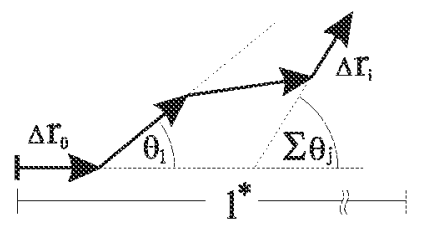

$$
l^{*}=\left\langle\sum_{i=0}^{\infty} \Delta r_{i} \cos \left(\sum_{j=0}^{i} \theta_{j}\right)\right\rangle=\ell \sum_{i=0}^{\infty}\langle\cos \theta\rangle^{i}=\frac{\ell}{1-\langle\cos \theta\rangle}
$$

where $\Delta r_{i}$ is the length of the $i$ 'th scattering event, $\ell$ the scattering mean free path and $\theta_{j}$ the scattering angle between two scattering events. $\left(\sum \theta_{j}\right)$ is the angle of the $i$ 'th scattering event with respect to $\Delta \vec{r}_{0}\left(\theta_{0} \equiv 0\right)$. The cosine represents the projection on $\overrightarrow{\Delta r}_{0}$. On average over all configurations the cosine of the sum of the angles can be transformed to a power of the averaged cosine of the scattering angle.

In fact, by assuming that the random walk starts at a depth $\ell^{\star}$ inside the turbid media, we have so far implicitly assumed that $l^{*}=\ell^{\star}$. With eq. (1.64) and the definition of $\ell^{\star}$ in section 1.1, we see that this is correct if the path length distribution for light scattering is exponential. However, for polymers with a constant segment length one obtains [21, 74]:

$$
\ell^{\star}=\ell \cdot \frac{1+\langle\cos \theta\rangle}{1-\langle\cos \theta\rangle}, \quad n^{\star}=n \cdot \frac{1-\langle\cos \theta\rangle}{1+\langle\cos \theta\rangle} \quad \text { but } \quad l^{*}=\frac{\ell}{1-\langle\cos \theta\rangle} .
$$

Precisely speaking, the relation $D=v_{E} \ell^{\star} / d$ (eq. 1.13) is only correct for an exponential step length distribution. For example in one dimension, for a constant step length distribution one can easily see that $\vec{\jmath}$ equals $-\frac{1}{2} v_{E} \ell^{\star} \vec{\nabla} \rho$ 
and not $-v_{E} \ell^{\star} \vec{\nabla} \rho$ (see section 1.1): At a point $x$ with density $\rho(x)$, from one moment to the next $\rho(x) / 2$ particles go to and $\rho(x+\Delta x) / 2$ particles come from the right. With $\Delta x=v_{E} \Delta t$ follows the statement. Thus, for consistency, we obtain in the case of a constant step length for the diffusion constant in one dimension $D_{1}^{\text {const }}=v_{E} \ell^{\star} / 2$ and not $D_{1}=v_{E} \ell^{\star}$.

We want to finish with a remark upon Rayleigh scattering. Rayleigh scattering is called isotropic although the form factor is not really isotropic but more 'doughnut'-like. In fact, the linear polarized coherent backscattering cone of Rayleigh scatterers is not rotationally invariant and thus not isotropic. However, in the multiple scattering regime, where the light propagation is diffuse, Rayleigh scattering is isotropic as $\langle\cos \theta\rangle=0$ and consequently $\ell^{\star}=\ell$.

\subsection{Appendix B: Proof of eqs. $(1.57,1.58)$}

In order to prove the relations between coherent backscattering and the remaining degree of polarization, the values for $\left|e_{\otimes}\right|^{2},\left|e_{\odot}\right|^{2}$ and $2 \Re\left[e_{\otimes} e_{\odot}^{*}\right]$ must be calculated for the different polarization states and orientations of the sample. The analyzer/polarizer pairs $[\vec{A}, \vec{P}]$ for the different cases were chosen according to Table 1.1. The vector $(1,-i)$ corresponds to a right-hand polarized wave. For circular polarization the orientation of the sample is of no importance because this case is rotational invariant in itself. With these choices, i.e. $\vec{A}=\vec{P}$ for the $(\|)$-cone and $\vec{A} \perp \vec{P}$ for the $(\perp)$-cone, the analyzer/polarizer pairs for the direct and reversed paths are identical. With $\mathbb{I M}=\left(\begin{array}{cc}-a & -b \\ c & d\end{array}\right)$ and the normalization $|a|^{2}+|b|^{2}+|c|^{2}+|d|^{2}=I_{o} \equiv 1$, one obtains the terms in Table 1.2. Obviously, some terms are different for the direct and reversed

\begin{tabular}{|c|c|c|c|c|}
\hline & {[} & & & \\
\hline$x x$ & {$\left[\left(\begin{array}{l}1 \\
0\end{array}\right),\left(\begin{array}{l}1 \\
0\end{array}\right)\right]$} & {$\left[\left(\begin{array}{l}0 \\
1\end{array}\right),\left(\begin{array}{l}0 \\
1\end{array}\right)\right]$} & $\frac{1}{\sqrt{2}}\left[\left(\begin{array}{c}1 \\
1\end{array}\right),\left(\begin{array}{l}1 \\
1\end{array}\right)\right]$ & $\frac{1}{\sqrt{2}}\left[\left(\begin{array}{c}-1 \\
1\end{array}\right),\left(\begin{array}{c}-1 \\
1\end{array}\right)\right]$ \\
\hline$x y$ & {$\left[\left(\begin{array}{l}0 \\
1\end{array}\right),\left(\begin{array}{l}1 \\
0\end{array}\right)\right]$} & {$\left[\left(\begin{array}{l}1 \\
0\end{array}\right),\left(\begin{array}{l}0 \\
1\end{array}\right)\right]$} & $\frac{1}{\sqrt{2}}\left[\left(\begin{array}{c}-1 \\
1\end{array}\right),\left(\begin{array}{l}1 \\
1\end{array}\right)\right]$ & $\frac{1}{\sqrt{2}}\left[\left(\begin{array}{c}1 \\
1\end{array}\right),\left(\begin{array}{c}-1 \\
1\end{array}\right)\right]$ \\
\hline++ & \multicolumn{3}{|c|}{$\frac{1}{\sqrt{2}}\left[\left(\begin{array}{c}1 \\
-i\end{array}\right),\left(\begin{array}{c}1 \\
-i\end{array}\right)\right]$} \\
\hline+- & $\frac{1}{\sqrt{2}}\left[\left(\begin{array}{c}1 \\
i\end{array}\right),\left(\begin{array}{c}1 \\
-i\end{array}\right)\right]$ \\
\hline
\end{tabular}

Table 1.1 


\begin{tabular}{|c|c|c|c|c|}
\hline & $\square$ & $\square$ & 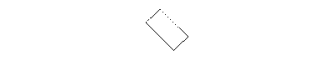 & $\gamma$ \\
\hline$\left|e_{\otimes}^{x x}\right|^{2}=\left|e_{\odot}^{x x}\right|^{2}$ & $|a|^{2}$ & $|d|^{2}$ & $\frac{1}{4}|a+b-c-d|^{2}$ & $\frac{1}{4}|a-b+c-d|^{2}$ \\
\hline$\left|e_{\otimes}^{x y}\right|^{2}$ & $|c|^{2}$ & $|b|^{2}$ & $\frac{1}{4}|a+b+c+d|^{2}$ & $\frac{1}{4}|a-b-c+d|^{2}$ \\
\hline$\left|e_{\odot}^{x y}\right|^{2}$ & $|b|^{2}$ & $|c|^{2}$ & $\frac{1}{4}|a-b-c+d|^{2}$ & $\frac{1}{4}|a+b+c+d|^{2}$ \\
\hline$\left|e_{\otimes}^{++}\right|^{2}=\left|e_{\odot}^{++}\right|^{2}$ & \multicolumn{4}{|c|}{$\left.\frac{1}{4}|(a+d)-i(b-c)|^{2}\right)$} \\
\hline$\left|e_{\otimes}^{+-}\right|^{2},\left(\left|e_{\odot}^{+-}\right|^{2}\right)$ & \multicolumn{4}{|c|}{$\left.\frac{1}{4}| \pm(a-d)-i(b+c)|^{2}\right)$} \\
\hline $\mathcal{P}_{\otimes}^{l}$ & $\frac{|a|^{2}-|c|^{2}}{|a|^{2}+|c|^{2}}$ & $\frac{|d|^{2}-|b|^{2}}{|d|^{2}+|b|^{2}}$ & $-\frac{\Re\left[(a+b)(c+d)^{*}\right]}{\frac{1}{2}+\Re\left[a b^{*}\right]+\Re\left[c d^{*}\right]}$ & $\frac{\Re\left[(a-b)(c-d)^{*}\right]}{\frac{1}{2}-\Re\left[a b^{*}\right]-\Re\left[c d^{*}\right]}$ \\
\hline $\mathcal{P}_{\odot}^{l}$ & $\frac{|a|^{2}-|b|^{2}}{|a|^{2}+|b|^{2}}$ & $\frac{|d|^{2}-|c|^{2}}{|d|^{2}+|c|^{2}}$ & $\frac{\Re\left[(a-c)(b-d)^{*}\right]}{\frac{1}{2}-\Re\left[a c^{*}\right]-\Re\left[b d^{*}\right]}$ & $-\frac{\Re\left[(a+c)(b+d)^{*}\right]}{\frac{1}{2}+\Re\left[a c^{*}\right]+\Re\left[b d^{*}\right]}$ \\
\hline $\mathcal{P}_{l}(\bigotimes)$ (see text) & \multicolumn{4}{|c|}{$2\left(|a|^{2}-|b|^{2}\right)$} \\
\hline $\mathcal{P}_{\otimes}^{c}$ & \multicolumn{4}{|c|}{$\frac{\Re\left[a d^{*}-b c^{*}\right]+\Im\left[a c^{*}+b d^{*}\right]}{\frac{1}{2}+\Im\left[b a^{*}+d c^{*}\right]}$} \\
\hline $\mathcal{P}_{\odot}^{c}$ & \multicolumn{4}{|c|}{$\frac{\Re\left[a d^{*}-b c^{*}\right]+\Im\left[b a^{*}+c d^{*}\right]}{\frac{1}{2}+\Im\left[a c^{*}+b d^{*}\right]}$} \\
\hline $2 \Re\left[e_{\otimes}^{x y}\left(e_{\odot}^{x y}\right)^{*}\right]$ & \multicolumn{2}{|c|}{$-2 \Re\left[b c^{*}\right]$} & \multicolumn{2}{|c|}{$\frac{1}{2}\left(|a+d|^{2}-|b+c|^{2}\right)$} \\
\hline $2 \Re\left[e_{\otimes}^{+-}\left(e_{\odot}^{+-}\right)^{*}\right]$ & \multicolumn{4}{|c|}{$\frac{1}{2}\left(|a-d|^{2}-|b+c|^{2}\right)$} \\
\hline
\end{tabular}

Table 1.2

paths. The assumption that, on average, both directions are identical results in some of the relations listed further below. Finally, with these expressions, equations $(1.57,1.58)$ can be verified.

In Table 1.2 also an expression for the linear depolarization $\mathcal{P}_{l}(\bigotimes)$ of a rotational invariant sample, i.e. of a rotational invariant matrix $M$, is indicated. This relation can be easily verified by the fact that in the case of rotational symmetry all elements in one row of Table 1.2 must be identical. In this way, also the following relations for a rotationally invariant matrix are found: $\left\langle|a|^{2}\right\rangle=\left\langle|d|^{2}\right\rangle,\left\langle|b|^{2}\right\rangle=\left\langle|c|^{2}\right\rangle,\left\langle a b^{*}\right\rangle=-\left\langle c d^{*}\right\rangle,\left\langle a c^{*}\right\rangle=-\left\langle b d^{*}\right\rangle$ and $\left\langle\Re\left[a d^{*}+b c^{*}\right]\right\rangle= \pm\left\langle|a|^{2}-|b|^{2}\right\rangle$. The plus/minus sign in the last relation is for the transmission/backscattering geometry, respectively. The average must be taken over all possible configurations. From Table 1.2 also other relations follow, e.g. with $0 \leq C B E^{x y} \leq 2$ follows $-\left\langle|b|^{2}\right\rangle \leq \Re\left[b c^{*}\right] \leq\left\langle|b|^{2}\right\rangle$.

One can show that for a succession of $n^{\star}$ independent identical scatterers, 
represented by $n^{\star}$ independent rotationally invariant matrixes $\mathrm{M}_{i}$, the remaining degree of polarization is given by $\mathcal{P}_{c}\left(n^{\star}\right)=\left\langle 2 \Re\left[a d^{*}-b c^{*}\right]\right\rangle^{n^{\star}}$ and $\mathcal{P}_{l}\left(n^{\star}\right)=$ $\left\langle 2\left(|a|^{2}-|b|^{2}\right)\right\rangle^{n^{\star}}$. Again, the average is taken over all possible configurations. However, in the case $\mathcal{P}_{l}\left(n^{\star}\right)$ the additional relation $\left\langle a b^{*}\right\rangle=\left\langle a c^{*}\right\rangle=0$ is necessary which (as can be shown) is equivalent to the condition that the medium has no optical activity. With $n^{\star}=s / \ell^{\star}$ follows for the characteristic depolarization length for the linear $(l)$ or circular $(c)$ polarization $\ell_{p}=-\ell^{\star} / \ln \left[\mathcal{P}_{l, c}^{n^{\star}}=\mathbf{1}\right]$ (see also text to eq. (1.52)). Once again we want to note that the matrixes $\mathbf{M}_{i}$ are supposed to be normalized by $|a|^{2}+|b|^{2}+|c|^{2}+|d|^{2}=I_{o} \equiv 1$.

\subsection{Appendix C: Monte-Carlo simulations}

The method of partial photons is correct for the following reason: At each scattering event the photon is 'divided' into a part $d \Omega$ which is scattered in the exact backscattering direction and a part $(1-d \Omega)$ which continues the random walk. With $d \Omega \rightarrow 0$, the probability for each 'partial

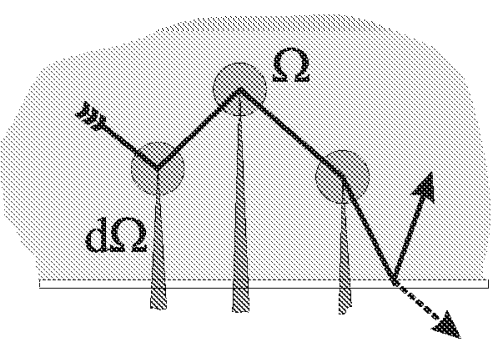
photon' to be scattered in the backscattering direction is the same (to the first order in $d \Omega$ ) and consequently only a normalization factor of no importance. The advantage of this method is that each scattering event, according to its depth $z$, gives a contribution to the result. Thus, a number of about ten times less photon trajectories (for isotropic scattering in backscattering geometry) are necessary compared to the 'classical' method in order to obtain the same precision. Especially in cases where the boundary conditions (describing how the light crosses the surface) are complicated, this is an important advantage as these boundary conditions must only be calculated now and again for the whole light path but not for every partial photon (again this would only be a non-important constant factor). The method of partial photons becomes less effective the larger the ratio $\ell^{\star} / \ell$ as only the part of the light path which is less than a distance of about $\ell$ away from the surface participates in the summation. Generally, in the case of anisotropic scattering, a factor of about $\ell^{\star} / \ell$ more scattering events must be to calculated for each light path. This increase in calculation time may be reduced by approaching the real, anisotropic scattering by isotropic scattering with the corresponding step length $\ell^{\star}$ at any time the light path is deeper 
in the sample than several length of $\ell^{\star}$. At this depth, the scattering can be considered completely diffusive.

We conclude this appendix with some technical remarks on the implementation of the probability distributions. In order to simulate, for example, the path length distribution $p(\Delta r)$, one has to find (analytically or numerically) the 'characteristic function' $P^{-1}(\eta)$ which is the inverse function of the indefinite integral $P=\int p(x) d x$ of the normalized distribution function itself. $P^{-1}(\eta)$ gives the correct probability distribution, $p(\Delta r)$ in this example, if $\eta$ is a random number between 0 and 1 . For the path length distribution one finds: $P^{-1}(\Delta r)=-\ell^{\star} \ln \eta$. In analogy, the characteristic function for the scattering can be found. However, this is more complicated normally, as the differential cross section depends on two, non-independent angles. Another possibility to choose one or more variables with the right probability distribution corresponds more to a trial and error technique: First the variables are chosen randomly (with the same probability within a certain interval), then the probability for this combination of variables is calculated and finally the random number generator decides, according to this calculated probability, whether this combination of variables is taken or not. The advantage of this method is that any probability distribution can be simulated, the disadvantage is that there may be many time-consuming bad trials. Mostly, it is advantageous to combine both methods and to select the variables in the trial and error method according to a 'pre-weighting' function such that the number of bad trials reduces to a minimum. For Rayleigh-Gans-Debye-scattering, for example, the part containing the matrix of Rayleigh-scattering can be simulated by the trial and error method; for the rest, which only depends on the scattering angle $\theta$, the function $P^{-1}$ can be determined numerically. Thus, the random selections of the angle $\theta$ are pre-weighted and the number of bad trials reduces to less than $50 \%$. With a trick, this method can also be applied to Mie-scattering by decomposing the scattering intensity into the two factors $f_{1}(\varphi, \theta) f_{2}(\theta)$ with $f_{1}(\varphi, \theta)=\left|E_{1}\right|^{2} \min \left(1, \frac{\left|S_{1}\right|^{2}}{\left|S_{2}\right|^{2}}\right)+\left|E_{2}\right|^{2} \min \left(1, \frac{\left|S_{2}\right|^{2}}{\left|S_{1}\right|^{2}}\right)$ and $f_{2}(\theta)=\max \left(\left|S_{1}\right|^{2},\left|S_{2}\right|^{2}\right)$. $E(\varphi)_{1,2}$ are the parts of the incident amplitude parallel and perpendicular to the scattering plain, $S(\theta)_{1,2}$ are the elements of the Mie-scattering matrix. The characteristic function $P^{-1}$ of $f_{2}$ can be found numerically, $f_{1}$ is evaluated by trial and error.

In order to create the random numbers $\eta$, the quality of implemented $\mathrm{C}$ or Fortran standard random number generators is sufficient as the scatterers are not located on a lattice but are distributed randomly. 


\subsection{Appendix D: Divergent or convergent light source}

For the coherent backscattering phenomena, the incident light need not necessarily be a plane wave $[75,76]$. Any light path starting and ending at the source will interfere constructively with its reversed path at the origin of the source. Thus, to some extent coherent backscattering acts like a time-reversal mirror. In the following we will calculate the cone width for non-parallel light (see fig. 1.26). Without the lens L1, the phase shift at point $x$ will be $\delta \phi=k r x / L+O[r, x]^{4} / L^{3}$, which is equal to the phase shift of parallel incident light and a lens of focal length $L$, corresponding to the 'classical'

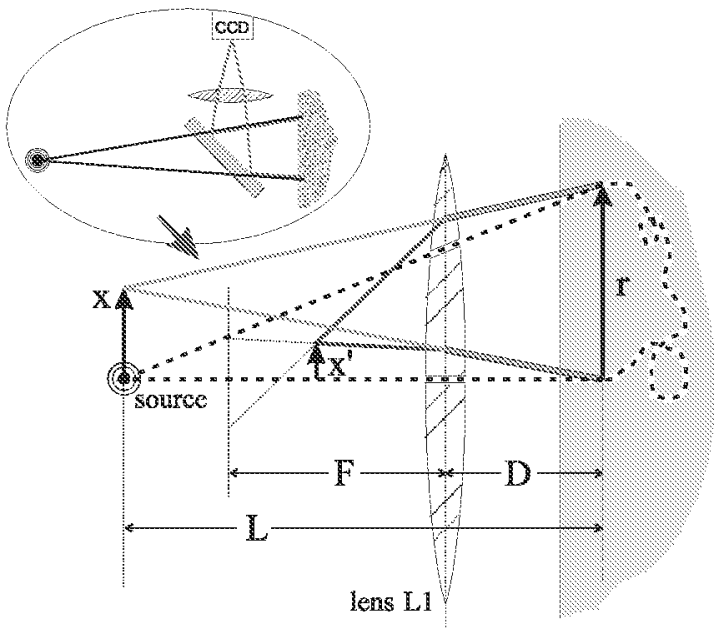

Figure 1.26: Sketch of an experimental setup with divergent incident light. For simplification, the semi-transparent mirror was omitted, therefore only the light which comes back from the sample 'sees' the lens $L 1$. setup. With lens L1, the point $x^{\prime}$ has the same phase shift as the point $x$ without lens. The ratio $x^{\prime} / x$ is given by

$$
\frac{x^{\prime}}{x}=\frac{F}{F+(L-D)}
$$

where we have used the fact that $x$ is the virtual image of $x^{\prime}$. Consequently, as the resolution at $x$ is proportional to $L$, the cone width $\Delta \theta_{\frac{1}{2}}$ at the point $x^{\prime}$ is proportional to $L x^{\prime} / x$ :

$$
\Delta \theta_{\frac{1}{2}} \propto \frac{F L}{F+(L-D)}
$$

With this formula, the scaling factor for any lens system can be calculated. For example, one obtains for the 'standard' setup (parallel incident light), in the case where a lens is put between the semi-transparent mirror and the sample, that the cone shape will rescale by the same amount as the diameter 
of the incident light spot is changed. ${ }^{17}$ Note, that the distance $D$ is not totally constant but fluctuates by about one $\ell^{\star}$. Therefore, $D$ should be much larger than $\ell^{\star}$.

Using divergent or convergent light may be an interesting variation of the experimental setup in order to choose the appropriate scaling factor. Of course, the position of the sample is then no longer indifferent.

\subsection{Appendix E: Strongly inclined samples and refraction at the surface}

If the sample is strongly inclined with respect to $\vec{k}_{i n}$, or if the coherent backscattering cone is very large, some assumptions made in section 1.3.1 are no longer correct. In these cases it is useful to separate the vectors $\vec{q}_{b}$ and $\vec{r}$ in a component parallel to the incident light (index ' $z$ ') and the components perpendicular to $\vec{k}_{i n}$, which for simplicity we will put together in one two-dimensional vector (index ' $x y$ ').

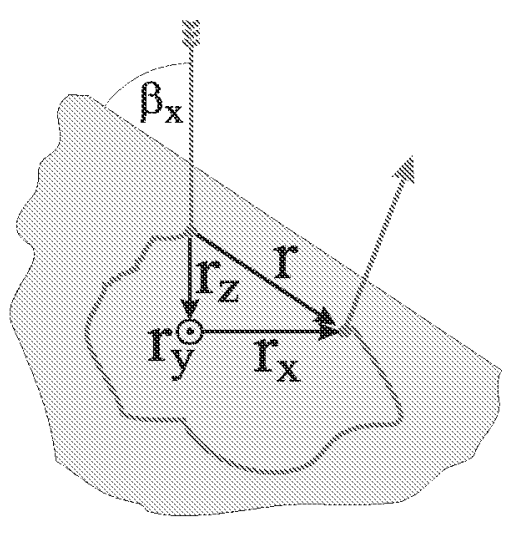
Note, that $\vec{r}_{x y}$ is now no longer parallel to the surface. With that notation one obtains:

$$
\vec{q}_{b}=\left(\begin{array}{c}
\vec{q}_{x y} \\
q_{z}
\end{array}\right)=\vec{k}_{\text {out }}-\left(-\vec{k}_{i n}\right)=\left(\begin{array}{c}
k_{o} \sin \theta_{b} \cdot \widehat{q}_{x y} \\
k_{o}\left(1-\cos \theta_{b}\right)
\end{array}\right) \stackrel{\theta_{b} \rightarrow 0}{\approx}\left(\begin{array}{c}
k_{o} \theta_{b} \widehat{q}_{x y} \\
k_{o} \theta_{b}^{2} / 2
\end{array}\right)
$$

where $\widehat{q}_{x y}=\vec{q}_{x y} /\left|q_{x y}\right|, k_{o}$ is the wave vector in air. The absolute value of the backscattering vector is $\left|q_{b}\right|=2 k_{o} \sin \left(\theta_{b} / 2\right) \stackrel{\theta_{b} \rightarrow 0}{\approx} k_{o} \theta_{b}$. Thus one obtains for the coherent part in eq. (1.40):

$$
\begin{aligned}
\cos \left(\vec{q}_{b} \cdot \vec{r}\right) & =\cos \left(\vec{q}_{x y} \cdot \vec{r}_{x y}\right) \cos \left(q_{z} r_{z}\right)-\sin \left(\vec{q}_{x y} \cdot \vec{r}_{x y}\right) \sin \left(q_{z} r_{z}\right) \\
\vec{q}_{x y} \cdot \vec{r}_{x y} & =k_{o} \sin \theta_{b}\left(\hat{q}_{x y} \cdot \vec{r}_{x y}\right) \approx k_{o} \theta_{b}\left(\widehat{q}_{x y} \cdot \vec{r}_{x y}\right) \\
q_{z} r_{z} & =k_{o}\left(1-\cos \theta_{b}\right) r_{z} \approx k_{o} \theta_{b}^{2} r_{z} / 2
\end{aligned}
$$

\footnotetext{
${ }^{17}$ For proof: In this case, the source is at the distance $L=D-f$ where $f$ (negative for concave lens) is the focal length of the supplementary lens between the semi-transparent mirror and the sample. For the detection system, i.e. for the backscattered light, both lenses must be added.
} 
where $\widehat{q}_{x y} \vec{r}_{x y}$ represents the projection of $\vec{r}_{x y}$ in the scattering plane, built up by $\vec{k}_{\text {out }}$ and $-\vec{k}_{i n}$. Consequently, the cone becomes broader in the direction of the inclination as $r_{x y}<r$. It also becomes asymmetric due to the sine terms which do not average out as $\vec{r}_{x y}$ and $r_{z}$ are correlated and have always the same sign. Thus, one obtains a modified eq. (1.46) for the cone in the direction of the inclination:

$$
C B E\left(q_{x}\right)=1+\mathcal{C}_{0} \frac{1-e^{-2\left(\sin \beta_{x}+\gamma\right) \ell^{\star} \sqrt{\left(q_{x} \sin \beta_{x}+q_{z} \cos \beta_{x}\right)^{2}}}}{2(\underbrace{\sin \beta_{x}}_{\substack{\text { reduced pene- } \\ \text { tration depth }}}+\gamma) \ell^{\star} \sqrt{\left(q_{x} \sin \beta_{x}+q_{z} \cos \beta_{x}\right)^{2}}}
$$

where $q_{x}$ and $q_{z}$ are given by eq. (1.68). The fact that the random walk starts at a reduced depth was also taken into account. ${ }^{18}$ Note that in the $y$-direction the cone shape is more or less unchanged although the penetration depth has changed. According to eq. (1.72), for small angles $\beta_{x}$, i.e. strong inclination, the cone becomes broader, asymmetric and there is a second maximum at the angle $2 \beta_{x}$. This second maximum, however, points into the sample. Moreover, it is more or less averaged out, as the vectors $\vec{r}$ and thus the angle $\beta$ fluctuate around the exact direction parallel to the surface. In addition, depending on the type of scatterers, it is not evident that the theorem of reciprocity is still valid in directions which differ strongly from the exact backscattering direction.

Due to the mismatch of the index of refraction at the surface, light is refracted when penetrating into and out of the sample (in addition, there are total internal reflections which may change $p(s)$ significantly, see section 1.3.4). The $q_{b}$-vector used in the formulas for $C B E$, is the wave vector in air. Of course, the light is refracted when crossing the interface sample/air and consequently, the corresponding

\footnotetext{
${ }^{18}$ In reality, the dependence of $p(\diamond)$ on the angle $\beta$ may be more complicated.
} 
backscattering angle in the sample is smaller by the value of the index of refraction (assuming more or less normal incidence) than outside of the sample; but the wavelength is reduced by the same factor such that $q_{b}$ is unchanged. However, if the sample is strongly inclined, there is an additional deformation due to refraction. From Fresnel laws the following relation follows between the width $d \beta_{2}$ of

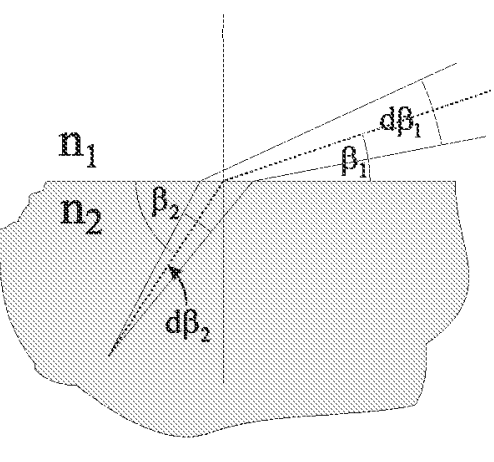
the cone inside the sample and outside the sample $d \beta_{1}$ :

$$
d \beta_{1}=\frac{n_{2}}{n_{1}} \frac{\sin \beta_{2}}{\sin \beta_{1}} d \beta_{2},
$$

where $n_{1}$ and $n_{2}$ are the corresponding indices of refraction. Consequently, due to refraction, the cone is wider ${ }^{19}$ by a factor $\sin \beta_{2} / \sin \beta_{1}$ in direction of the inclination as opposed to perpendicular to it. Investigations in this direction may be useful for a better understanding of the influence of the interface on the light propagation in strongly multiple scattering samples.

\footnotetext{
${ }^{19}$ In addition to the deformation calculated above. The factor $n_{2} / n_{1}$ is compensated for by the difference in the wavelength inside and outside the sample.
} 


\section{Bibliography}

[1] Schuster, A.; Radiation through a foggy atmosphere; Astrophys. J. 21, 1 (1905)

[2] Schwarzschild, K.; Über das Gleichgewicht der Sonnenatmosphaere; Nachrichten der Königlichen Gesellschaft der Wissenschaften zu Göttingen, Mathematisch-physikalische Klasse, 41 (1906)

[3] P. M. Morse, H. Feshbach; Methods of Theoretical Physics, McGraw-Hill Book Company, New York, (1953)

[4] Dainty, J.C.; Laser Speckle and Related Phenomena; Topics in Applied Physics 9, Springer-Verlag, Heidelberg, (1984)

[5] Weitz, D.A.; Pine, D.J.; Dynamic Light Scattering; edited by Brown W., Clarendon Press, Oxford (1993) 652-720

[6] Bergmann, G.; Weak localization in thin films. A time-of-flight experiment with conduction electrons; Phys. Rep. 107, 1 (1984) 1-58

[7] Adams, P.W.; Paalanen, M.A; Localization in a Nondegenerate TwoDimensional Electron Gas; Phys. Rev. Lett. 58, 20 (1987) 2106-2109

[8] Anderson, P.W.; Absence of Diffusion in Certain Random Lattices; Phys. Rev. 109, (1958) 1492

[9] Anderson, P.W.; The question of classical localization. A theory of white paint?; Philosophical Magazine B 52, 3 (1985) 505-509

[10] Wiersma, D.S.; Bartolini, P.; Lagendijk, A.; Righini, R.; Localization of light in a disordered medium.; Nature 390, 6661 (1997) 671-673

[11] Scheffold, F.; Lenke, R.; Tweer, R. and Maret, G.; Localization or classical diffusion of light?, Nature 398, 207 
[12] Sheng, P. (editor); Scattering and Localization of Classical Waves in Random Media, World Scientific, Singapore (1990)

[13] Genack, A.Z.; Fluctuations, Correlation and Average Transport of Electromagnetic Radiation in Random Media, in [12]

[14] Sheng, P.; Introduction to Wave Scattering, Localization, and Mesoscopic Phenomena, Academic Press, San Diego (1995)

[15] van Albada, M.P.; Lagendijk, A.; Observation of Weak Localization of Light in a Random Medium; Phys. Rev. Lett. 55, 24 (1985) 2692-2695

[16] Wolf, P.-E.; Maret, G.; Weak Localization and Coherent Backscattering of photons in Disordered Media; Phys. Rev. Lett. 55, 24 (1985) 2696-2699

[17] Bryant, H.C.; Nelson J.; The Glory; Scientific American, (July 1974) 6071

[18] Mack, U.; Lenke, R.; Maret, G,; Comparison between 'The Glory' and Coherent Backscattering of Light in Random Media; submitted to Phys. Rev. E

[19] van de Hulst, H.C.; Light Scattering by Small Particles, Dover Publications, New York, (1957)

[20] Fraden, S.; Maret G.; Multiple Light Scattering from Concentrated, Interacting Particles; Phys. Rev. Lett. 65 4, (1990) 512-515

[21] Doi,M.; Edwards, S.F.; The Theory of Polymer Dynamics, Clarendon Press, Oxford (1989)

[22] Ishimaru, A.; Wave Propagation and Scattering in Random Media, Academic Press Inc., San Diego, (1978)

[23] Chandrasekhar, S.;Radiative Transfer, Dover Publications Inc., New York (1960)

[24] Amic, E.; Luck, J.M.; Nieuwenhuizen, T.M.; Multiple Rayleigh scattering of electromagnetic waves; J. Phys. I France 7, 3 (1997) 445-483

[25] de Bruijn, N.G.; Asymptotical methods in analysis; North-Holland Publishing Co., Amsterdam (1961) 
[26] Bronstein, I.N.; Semendjajew, K.A.;Taschenbuch der Mathematik;

[27] van Albada, M.P.; van Tiggelen, B.A.; Lagendijk, A.; Tip, A.; Speed of Propagation of Classical Waves in Strongly Scattering Media; Phys. Rev. Lett. 66, 24 (1991) 3132-3135

[28] van Tiggelen, B.A.; Lagendijk, A.; van Albada, M.P.; Tip, A.; Speed of light in random media; Phys. Rev. B 45, 21 (1992) 12233-12243

[29] van Tiggelen, B.A.; Lagendijk, A.; Rigorous treatment of the speed of diffusing classical waves; Europhys. Lett. 23, 5 (1993) 311-316

[30] Busch, K.; Soukoulis, C.M.; Transport Properties of Random Media: A New Effective Medium Theory; Phys. Rev. Lett. 75, 19 (1995) 3442-3445

[31] Schriemer, H.P.;Cowan, M.L.; Page, J.H.; Sheng, P.; Liu, Z.; Weitz, D.A.; Energy Velocity of Diffusing Waves in Strongly Scattering Media; Phys. Rev. Lett. 79, 17 (1997) 3166-3169

[32] Applications of MCP-PMT's to Time Correlated Single Photon Counting and Related Procedures; HAMAMATSU No. ET-03

[33] Born, M.; Wolf, E.; Principles of Optics, Pergamon Press (1959)

[34] Yodh, A; Chance, B.; Spectroscopy and Imaging with Diffusing Light; Physics Today, March 1995

[35] Jackson, J.D.; Classical Electrodynamics, John Wiley \& Sons, New York, (1962)

[36] Golubentsev, A.A.; Suppression of interference effects in multiple scattering of light; Sov. Phys. JETP 59, 1 (1984) 26-32

[37] MacKintosh, F.C.; John, S.; Coherent backscattering of light in the presence of time-reversal-noninvariant and parity-nonconserving media; Phys. Rev. B 37, 4 (1988) 1884-1897

[38] Erbacher, F.A.; Lenke, R.; Maret, G.; Multiple light scattering in magneto-optically active media; Europhys. Lett. 21, 5 (1993) 551-556

[39] Lenke, R.; Maret, G.; Affecting weak light localization by strong magnetic fields; Phys. Scr. T49B, (1993) 605-609 
[40] Lenke, R.; Maret, G.; Destruction of Optical Coherent Backscattering by Magnetic Faraday Rotation; OSA Proceedings on Advances in Optical Imaging and Photon Migration 21, R.R. Alfano (ed.), Optical Society of America (1994) 16-19

[41] Saxon, D.S.; Tensor Scattering Matrix for the Electromagnetic Field; Phys. Rev. 100, 6 (1955) 1771-1775

[42] Mishchenko, M.I.; Coherent backscattering by two-sphere clusters; Optics Lett. 21, 9 (1996) 623-625

[43] Wolf, P.E.; Maret, G.; Akkermans, E.; Maynard, R.; Optical coherent backscattering by random media: an experimental study; J. Phys. France 49, 1 (1988) 63-75

[44] Akkermans, E.; Wolf, P.E.; Maynard, R.; Maret, G.; Theoretical study of the coherent backscattering of light by disordered media; J. Phys. France 49, 1 (1988) 77-98

[45] Igarashi, J.; Coherent backscattering of neutrons; Phys. Rev. B 35, 16 (1987) 8894-8897

[46] Heiderich, A.; Diffusion Multiple en milieu non linéaire ou anisotrope; Thesis, Université Joseph Fourier-Grenoble I, France (1995)

[47] Mishchenko, M.I.; Enhanced backscattering of polarized light from discrete random media: calculations in exactly the backscattering direction; J. Opt. Soc. Am. A 9, 6 (1992) 978-982

[48] Ospeck, M.; Fraden, S.; Influence of reflecting boundaries and finite interfacial thickness on the coherent backscattering cone; Phys. Rev. E 49, 5 pt.B (1994) 4578-4589

[49] Wiersma, D.S.; van Albada, M.P.; Lagendijk, A.; An accurate technique to record the angular distribution of backscattered light; Rev. Sci. Instrum. 66, 12 (1995) 5473-5476

[50] Pitter, M.; Jakeman, E.; Harris, M.; Heterodyne detection of enhanced backscatter; Optics Lett. 22, 6 (1997) 393-395 
[51] Schirrer, R.; Lenke, R.; Boudouaz, J.; Study of mechanical damage in rubber-toughened poly (methylmethacrylate) by single and multiple scattering of light; Polymer Engineering and Science 37, 10 (1997) 1748-1760

[52] Vithana, H.K.M.; Asfaw, L.; Johnson, D.L.; Coherent Backscattering of Light in a Nematic Liquid Crystal; Phys. Rev. Lett. 70, 23 (1993) 35613564

[53] Heiderich, A.; Maynard, R.; van Tiggelen, B.A.; Coherent backscattering in nonlinear media; Optics Communications 115, 3-4 (1995) 392-400

[54] Dogariu, A.; Uozumi, J.; Asakura, T.; Enhancement of backscattered intensity from fractal aggregates; Waves in Random Media 2 (1992) 259-263

[55] Dogariu, A.; Uozumi, J.; Asakura, T.; Enhancement Factor in the Light Backscattered by Fractal Aggregated Media; Optical Review 3, 2 (1996) 71-82

[56] Ishii, K.; Iwai, T.; Asakura, T.; Polarization properties of the enhanced backscattering of light from the fractal aggregate of particles; Optical Review 4, 6 (1997) 643-647

[57] Maradudin, A.A.; Nieto-Vesperinas, M.; Thorsos, E.I.; Enhanced backscattering of light from randomly rough surfaces and related phenomena II. Two-dimensional surfaces and localization of surface plasmon polaritons; Comments on Condensed Matter Physics 17, 2 (1994) 59-78

[58] Maradudin, A.A.; Nieto-Vesperinas, M.; Thorsos, E.I.; Enhanced backscattering of light from randomly rough surfaces and related phenomena. I. One-dimensional surfaces and angular correlation functions of scattered fields; Comments on Condensed Matter Physics 17, 1 (1994) 13-37

[59] Wiersma, D.S.; van Albada, M.P.; Lagendijk, A.; Coherent Backscattering of Light from Amplifying Random Media; Phys. Rev. Lett. 75, 9 (1995) 1739-1742

[60] de Oliveira, P.C.; Perkins, A.E.; Lawandy, N.M.; Coherent backscattering from high-gain scattering media; Optics Letters 21, 20 (1996) 1685-1687 
[61] Vreeker, R.; Van Albada, M.P.; Sprik, R.; Lagendijk, A.; Femtosecond time-resolved measurements of weak localization of light; Phys. Lett. A 132, 1 (1988) 51-54

[62] Lagendijk, A.; Vreeker, R.; De Vries, P.; Influence of internal reflection on diffusive transport in strongly scattering media; Phys. Lett. A 136, 1-2 (1989) 81-88

[63] Saulnier, P.M.; Watson, G.H.; Role of surface reflectivity in coherent backscattering measurements; Optics Lett. 17, 13 (1992) 946-948

[64] den Outer, P.N.; Lagendijk, A.; Influence of the refractive index contrast on coherent backscattering Optics Communications, 103, 3-4 (1993) 169173

[65] Bayer, G.; Niederdrank, T.; Weak localization of acoustic waves in strongly scattering media; Phys. Rev. Lett. 70, 25 (1993) 3884-3887

[66] Tourin, A.; Derode, A.; Roux, P.; van Tiggelen, B.A.; Fink, M.; TimeDependent Coherent Backscattering of Acoustic Waves; Phys. Rev. Lett. 79, 19 (1997) 3637-3639

[67] Lenke, R.; Maret, G,; Destruction of Coherent backscattering of Light by Magneto-Optical Faraday Rotation; to be published

[68] Lehner, R; Lenke, R.; Maret, G,; Influence of Magneto-Optical Faraday Rotation on Coherent backscattering in the case of Mie-Scattering; to be published

[69] Okamoto, T.; Asakura, T.; Enhanced backscattering of partially coherent light; Optics Lett. 21, 6 (1996) 369-371

[70] Mishchenko, M.I.; On the nature of the polarization opposition effect exhibited by Saturn's rings; Astrophysical Journal 411, 1 (1993) 351-361

[71] Mishchenko, M.I.; Diffuse and coherent backscattering by discrete random media. I. Radar reflectivity, polarization ratios, and enhancement factors for a half-space of polydisperse, nonabsorbing and absorbing spherical particles; J. Quant. Spectrosc. Radiat. Transfer 56, 5 (1996) 673-702 
[72] Rosenbush, V.K.; Avramchuk, V.V.; Rosenbush, E.; Mishchenko, M.I.; Polarization properties of the Galilean satellites of Jupiter: observations and preliminary analysis; Astrophys. J. 487, 1, pt.1 (1997) 402-414

[73] Hapke, B.W.; Nelson, R.M.; Smythe, W.D.; The Opposition Effect of the Moon: The Contribution of Coherent Backscatter; Science 260, (1993) $509-511$

[74] A. Heiderich, A. S. Martinez, R. Maynard, B. A. van Tiggelen, The role of step length distribution in wave-diffusion, Phys. Let. A 185, (1994) 110

[75] Dogariu, A.; Boreman, G.D.; Enhanced backscattering in a convergingbeam configuration; Optics Lett. 21, 21 (1996) 1718-1720

[76] Lenke, R.; Maret G.; Coherent backscattering of light in multiple scattering media; Progr Colloid Polym Sci 104, (1997) 126-128 